EPR analysis of SNARE proteins: transient states of the neuronal acceptor complex

\author{
Damian Dawidowski \\ Charlottesville, Virginia
}

M.S., Jagiellonian University, 2009

A Thesis presented to the Graduate Faculty of the University of Virginia in Candidacy for the Degree of

Doctor of Philosophy

\title{
Department of Chemistry
}

\section{University of Virginia}

May, 2014 


\section{Acknowledgments}

The following work was done in the research group of Prof. David S. Cafiso, at the Chemistry Department of The University of Virginia.

I would like to give my thanks to David Cafiso for his kindness, patience and his help during my entire doctoral program. Also I want to thank all the members of his lab, but particularly Dawn Herrick and WeiWei Kuo, for their much needed support in planning and executing scientific research.

I am grateful to Binyong Liang for his willingness to participate in a collaborative research project.

I would also like to thank Jeff Ellena for his assistance in executing NMR experiments and assisting me with computational analysis. 
Table of contents

Acknowledgments..............................................................................................................................II

Table of contents..................................................................................................................

Abbreviations ..................................................................................................................................

Abstract............................................................................................................................... VII

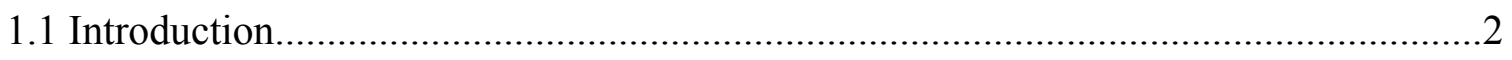

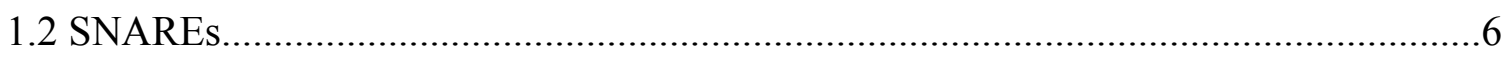

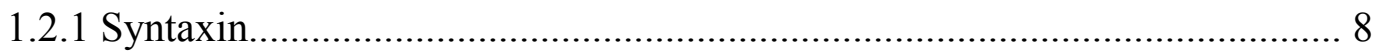

1.2.2 SNAP25

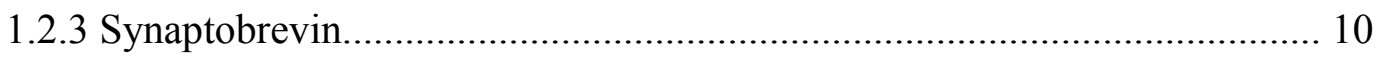

1.3 Binary syntaxin:SNAP25 interaction................................................................ 12

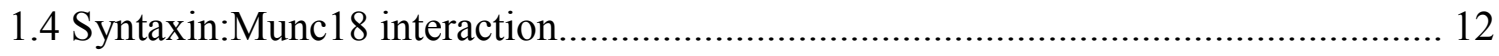

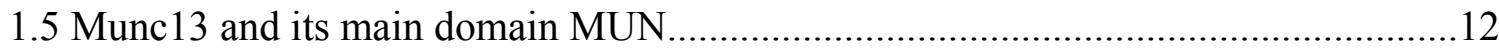

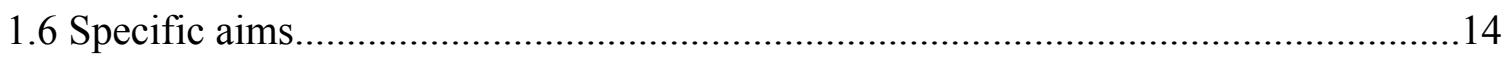

Chapter 2: Electron Paramagnetic Resonance (EPR)....................................................... 16

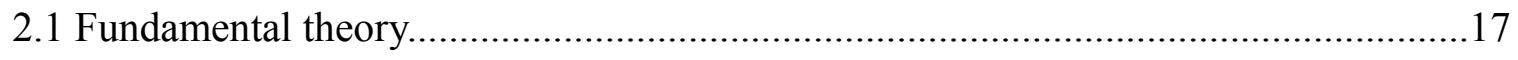

2.2 Continuous Wave EPR (CW EPR) ................................................................. 19

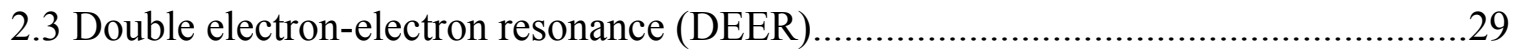

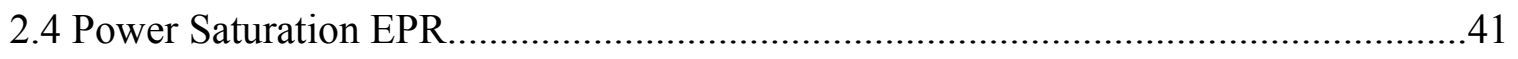

Chapter 3: Allosteric Control of Syntaxin 1a by Munc18-1: Characterization of the Open and Closed Conformations of Syntaxin (Dawidowski D, Cafiso D. Biophysical Journal, Volume 104, 1585-1594 (2013)............................................................................47 


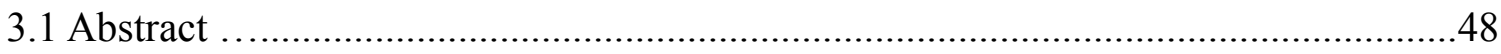

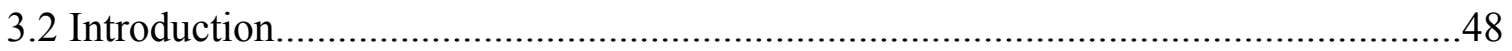

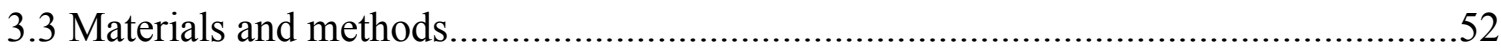

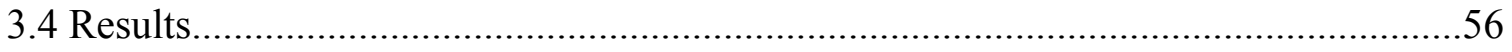

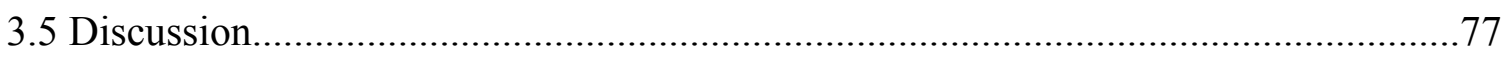



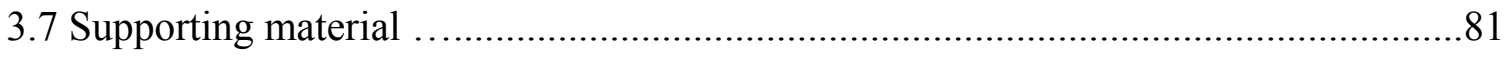

Chapter 4: The SNARE Motif of Synaptobrevin Exhibits an Aqueous-Interfacial Partitioning That Is Modulated by Membrane Curvature (Liang B., Dawidowski D., et al. Biochemistry, Volume 53, 1485-1494 (2014).................................................93

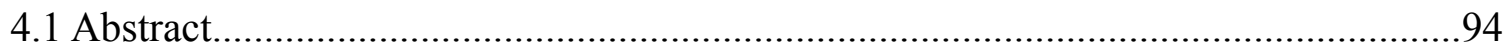

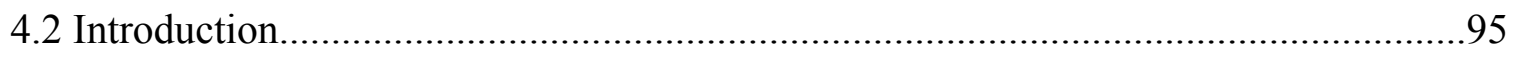

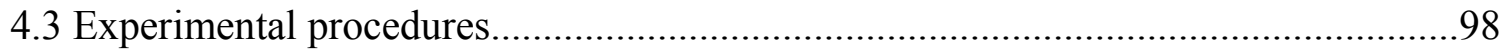

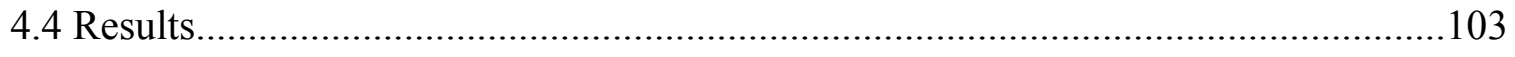

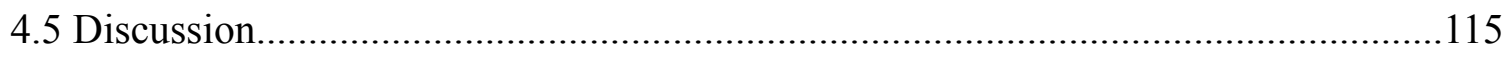

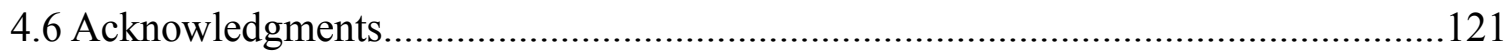

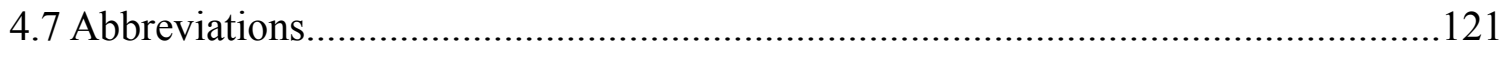

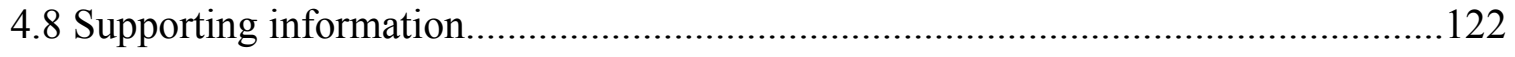

Chapter 5: Significance and Future Directions................................................................129

References......................................................................................................................133 
Abbreviations

AEBSF - 4-(2-Aminoethyl) Benzenesulfonyl Fluoride

CW - Continuous Wave

CD - Circular Dichroism

Da - Dalton

DEER - Double Electron Electron Resonance

DHPC - 1,2-Dihexanoyl-sn-Glycero-3-Phosphocholine

DMPC - 1,2-Dimyristoyl-sn-Glycero-3-Phosphocholine

DPC - Dodecylphosphocholine

DTT - Dithiothreitol

EDTA - Ethylenediaminetetraacetic Acid

EPR - Electron Paramagnetic Resonance

FID - Free Induction Decay

FRET - Förster resonance energy transfer

IPTG - Isopropyl $\beta$-D-1-thiogalactopyranoside

MOMD - Microscopic Order Macroscopic Disorder

MTSL - Methanethiosulfonate

MUNC-18 - Mammalian Uncoordinated-18

M.W. - Molecular Weight

MWCO - Molecular Weight Cut Off

NiEDDA - Nickel_Ethylenediammonium Diacetate

NiNTA - Nickel Nitrilotriacetic Acid 
NMR - Nuclear Magnetic Resonance

NSF - N-ethylmaleimide-sensitive factor

PBS - Phosphate Buffered Saline

PCR - Polymerase Chain Reaction

$\mathrm{SM}-\mathrm{Sec} 1 \mathrm{p} / \mathrm{Munc} 18$

SNARE - Soluble NSF (N-ethylmaleimide-sensitive factor) Attachment Protein Receptor Proteins

VAMP - Vesicle Associated Membrane Protein 


\section{Abstract}

Neuronal fusion involves complex protein machinery that acts in a highly orchestrated manner when triggered by a neuronal action potential. This process involves three SNAREs, a highly conserved group of proteins that can interact to form a coiled-coil assembly called the SNARE complex, which is believed to provide the driving force for fusion of the neurotransmitter-carrying vesicle to the presynaptic space. Aside from SNAREs, other proteins, such as Munc13, synaptotagmin and Munc18 serve regulatory roles by acting on SNAREs. The study presented here used EPR spectroscopy to analyze the structure and interactions of syntaxin, SNAP-25, synaptobrevin (the three SNAREs), Munc18 and the MUN domain of Munc13. By performing a detailed CW spectral analysis, it was discovered that both syntaxin and synaptobrevin exist in conformational equilibria between ordered and disordered states. The structural equilibrium of syntaxin is also modulated by Munc18, likely controlling its interaction with other SNAREs. Pulsed EPR studies also revealed syntaxin as existing predominantly in a close tertiary structure, and that Munc18 further stabilizes this closed state. Munc18 also acts to suppress the syntaxin-SNAP25 binary complex, which is a dead-end configuration for SNAREmediated fusion. Taking all the EPR data together, the SNAREs are shown as engaging in complex interactions with Munc18 and MUN, which results in an intricate pathway of numerous protein structural states and transient intermolecular interactions. 
Chapter 1: Neuronal Fusion 


\subsection{INTRODUCTION}

Information flow in the neuronal network depends upon action potentials, which travel along the neuron, until they reach the synapse. Neurotransmitter is released into the synapse, which initiates a signal in the postsynaptic neuron [1]. This release requires tight biomolecular control to ensure that a signal is created spontaneously, but only after a relevant receptor stimuli.

The neurotransmitter is carried in a lipid vesicle, that can fuse with the presynaptic membrane to allow this biomolecule to be released into the synaptic cleft. Afterwards, its binding to a specific receptor may propagate the action potential in the postsynaptic neuron. As shown in Figure 1, the carrier vesicles go through a cycle, being first filled with the neurotransmitter, then docked to the presynaptic membrane and eventually fusing with the membrane. Membrane endocytosis then recreates the vesicle pool.

The fusion of two lipid membranes, which is required for neurotransmitter release, is a universal mechanism occurring in cellular compartments of all Eukaryotes that allows for efficient communication across the cell. Is is believed to happen through a sequence of few separate events [2]. Initially, the two bilayers come into a close contact, which likely involves removal of some of nearby water molecules, that would otherwise oppose lipid fusion. Next, one of the lipid surfaced would exhibit a destabilization, where some of the lipid acyl chains flip to the side of phosphate head groups. Through this local effect the two lipid layers would come in contact and subsequently merge to form a hemifusion intermediate [3]. Subsequent fusion of the outer leaflets would then lead to full fusion (the chain of events is shown in Figure 2). 


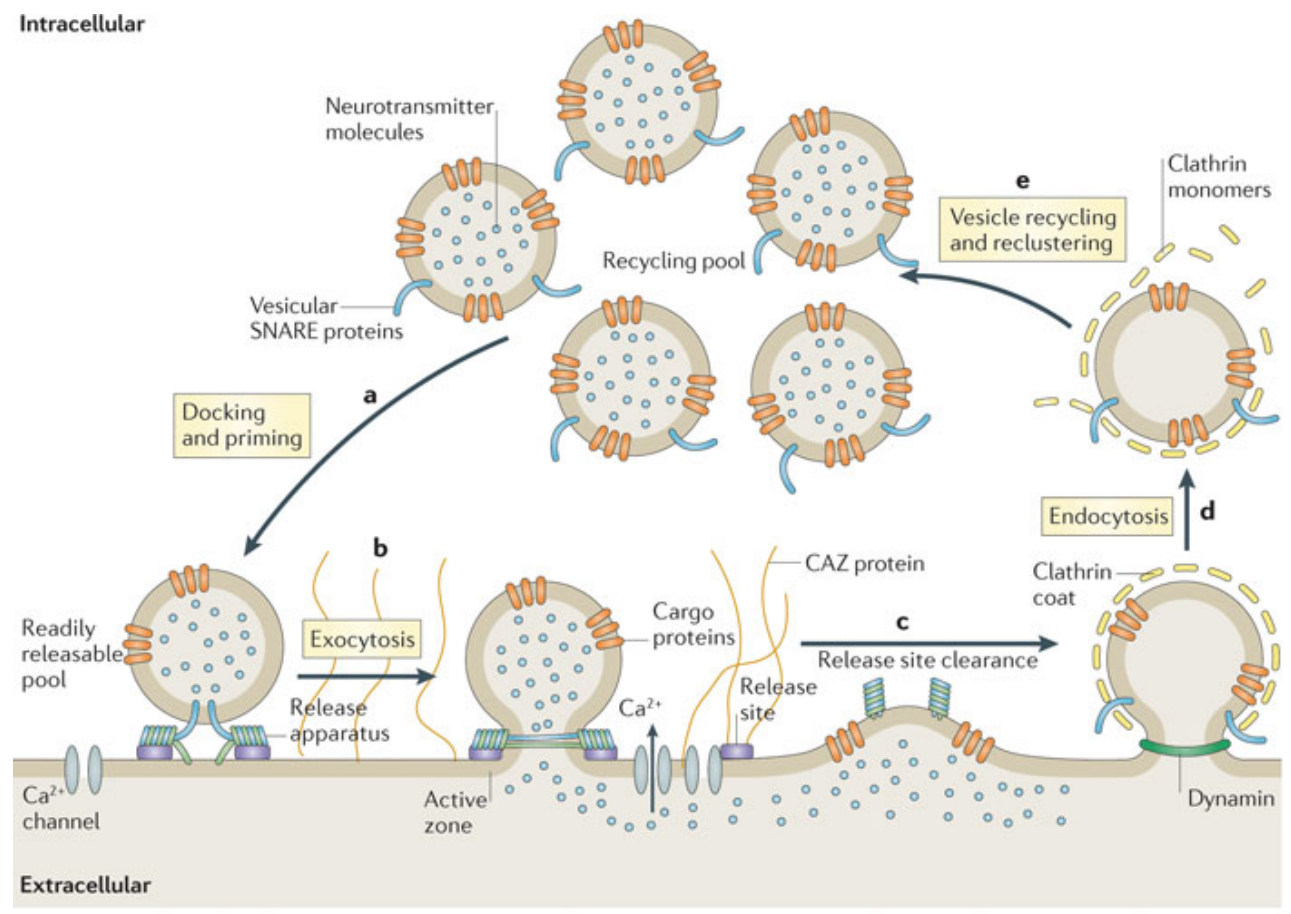

Nature Reviews | Neuroscience

Figure 1. A schematic representation of the neuronal fusion cycle. The fusion process starts with lipid vesicle "docking", which brings the vesicle in direct contact with the presynaptic membrane. (a) Here it adds to the pool of vesicles that can respond to the calcium trigger and fuse with the membrane (Readily Releasable Pool). It is then followed by exocytosis of the vesicle to the presynaptic space (b), which involves the vesicle fusing with the presynaptic membrane. The lipid surface around the fusion site is then cleared (c) by the NSF-dependent disassembly of the SNARE complex, and the vesicle is reformed through the endocytosis of presynaptic membrane (d), and then filled with neurotransmitter after which it rejoins the pool of vesicles in the cytosol (e) Figure taken from reference 4. 


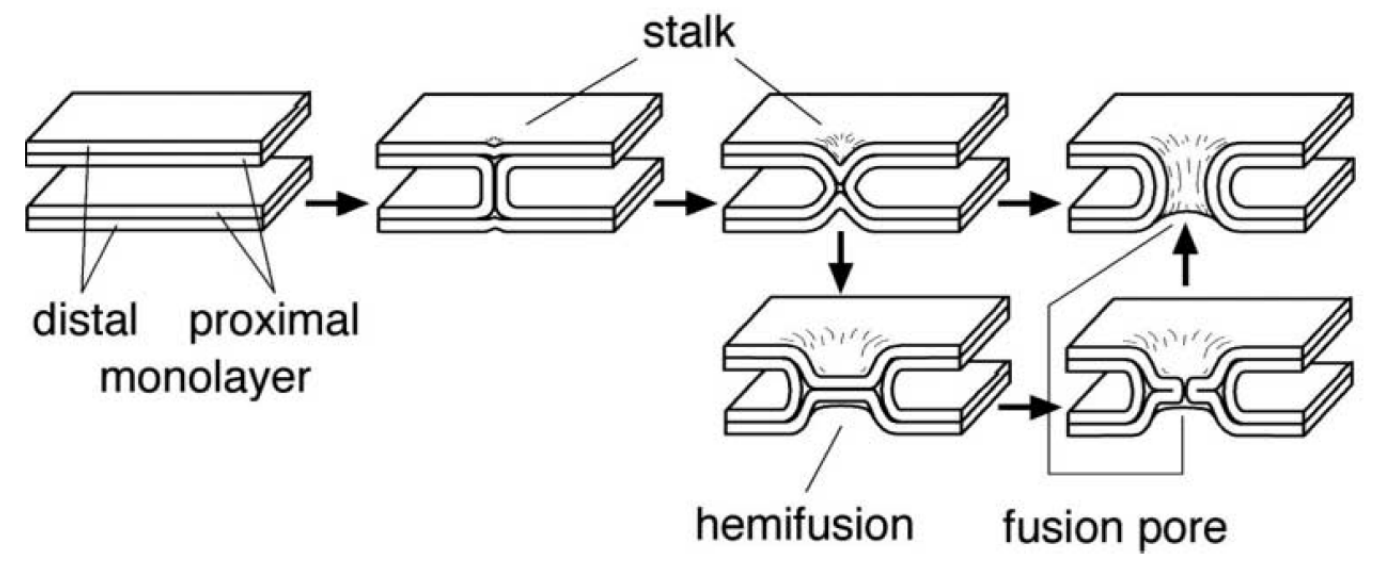

Figure 2. Stalk hypothesis for the mechanism of lipid fusion.

According to the model fusion is initiated by an hourglass-shaped contact between the two layers, termed the stalk, that then progresses into a hemifusion state and finally into a fusion pore. Figure taken from reference 98. 
It is widely accepted that the neuronal vesicle fusion mechanism is triggered by the rise of the intracellular calcium ion concentration, initiated by the action potential that opens voltage-sensitive calcium channels [5-8]. Once the process is started, the vesicle needs to overcome an energy barrier that opposes spontaneous fusion of two lipid bilayers $[9,10]$. In vitro studies show that such barrier makes the spontaneous lipid fusion a fairly slow process, with the fusion rate on the order of seconds [11]. Strikingly, the in vivo neuronal fusion mechanism exhibits very fast kinetics, with full vesicle fusion happening in less then 1 millisecond after the calcium trigger. This fact, along with precise calcium triggering, indicates that the neuronal fusion process requires an additional biomolecular machinery that would supplement the natural propensity of lipid bilayers to fuse [12].

It is generally agreed that the role of the SNARE proteins is to provide the forces necessary for lipid fusion [13, 14]. SNAREs are a group of highly conserved protein families that are involved in the fusion mechanisms across the entire cell, such as a lysosome merging with a phagosome [14] or in Golgi transport [15]. Aside from SNAREs, studies have also discovered several auxiliary proteins, that are required for efficient $\mathrm{Ca}^{2+}$ triggered fusion. Arguably the most studied one is synaptotagmin, which is widely considered to be the calcium sensor of neuronal fusion $[16,17]$. It is able to bind to the lipid bilayer with its two $\mathrm{C} 2$ domains in a calcium dependent fashion [18] and perhaps also have an effect on SNARE zippering [19, 20] or bridging the two bilayers closer to allow them to fuse [21]. The other regulatory proteins are Munc13, Munc18 and complexin. They are more likely to act through direct binding to either individual SNAREs or the SNARE complex [22]. 


\subsection{SNARES}

The name of the SNARE proteins is derived from soluble (N-ethylmaleimide sensitive factor (NSF) a attachment protein receptor. The importance of SNAREs in neuronal fusion was revealed because these proteins are the target of clostridium toxins that impair communication between neurons $[23,24]$. There are three neuronal SNAREs. Two proteins, syntaxin-1A and SNAP25, are located on the presynaptic membrane, while the third, called synaptobrevin (also named VAMP-2), is localized on the surface of the neurotransmitter carrying vesicle. All three proteins possess highly conserved 60-70 amino acid segments called the SNARE motifs; through these domains SNAREs can interact to form a coiled-coil tetra-helical bundle called the SNARE complex $[25,26]$. This complex has been successfully crystallized [27] and its structure is shown in Figure 3.

Initially, the role of SNAREs in fusion was believed to be the NSF-dependent disassembly of their SNARE complex, that would trigger the fusion event [28]. However, later studies have demonstrated that NSF disassembly is independent on fusion initiation and that the SNARE complex formation promotes fusion [29]. It is proposed that the formation of this complex starts from the N-termini of the SNARE motifs of the three SNARE proteins, which then proceeds to their C-terminus. This process, termed SNARE zippering, provides the forces that bring the opposite membranes closer together and force them to fuse [30]. It is also proposed, that the SNARE zippering carries through to the transmembrane segments of syntaxin and synaptobrevin, impacting the bilayers directly, and perhaps contributing to their distortion that would stimulate lipid fusion [31].

The SNARE motifs are highly conserved and feature numerous hydrophobic 


\section{$\mathbf{a}$

\begin{tabular}{ccc} 
Synaptobrevin & \\
\cline { 2 - 2 } & SNARE TM \\
motif
\end{tabular}

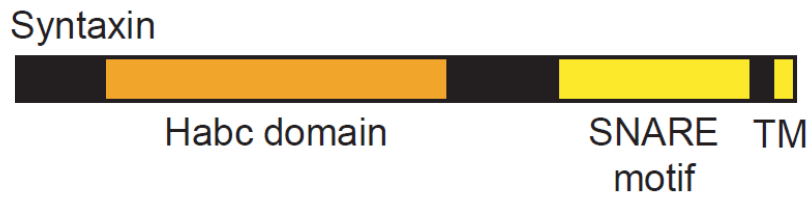

SNAP-25

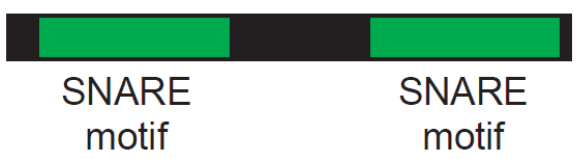

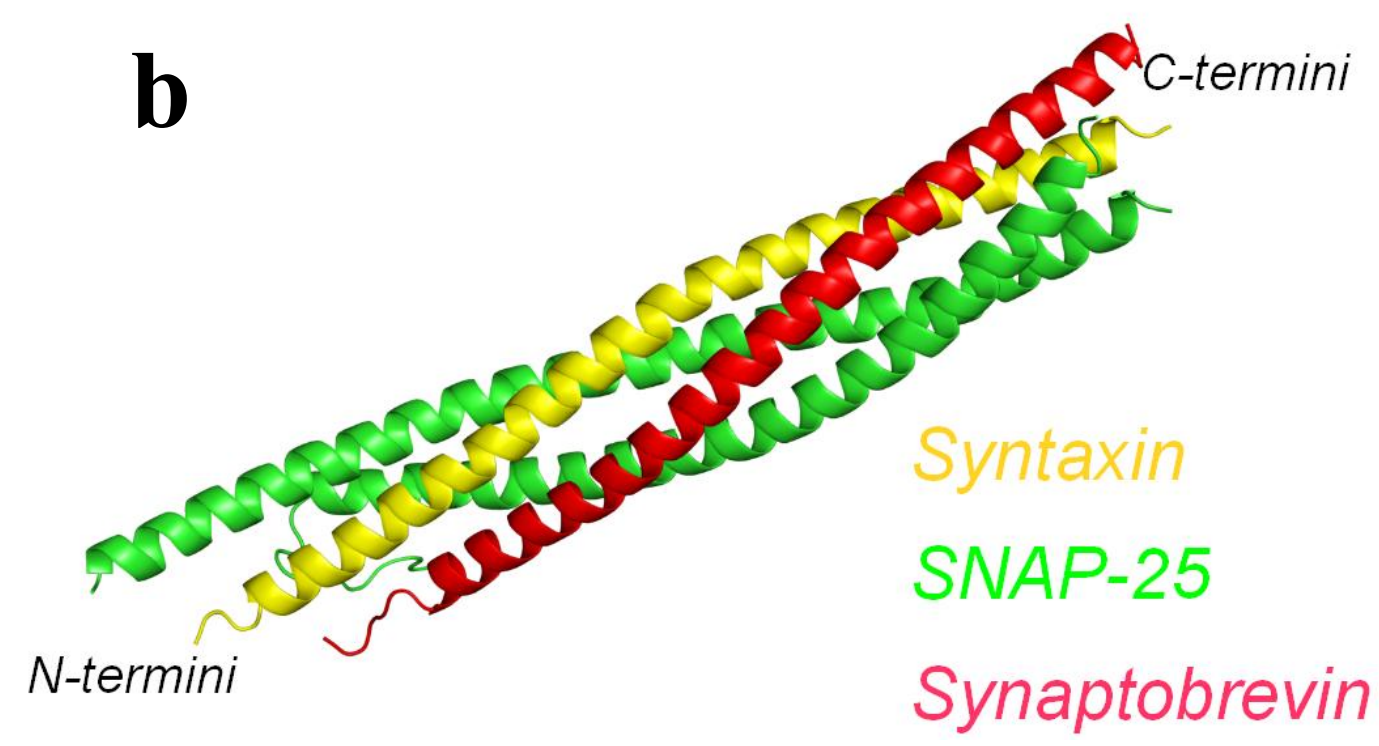

Figure 3. a) Schematic representation of the structure of three neuronal SNAREs. SNAREs contain the SNARE motifs that participate in the SNARE complex formation. Figure adopted from reference 75 . b) The crystal structure of the SNARE complex. The SNARE domains of Syntaxin, SNAP25 and synaptobrevin form a four-helix bundle called the SNARE complex. [27]. Figure made with PyMOL (DeLano Scientific LLC, Palo Alto, CA). PDB accession code: 1SFC. 
amino acids which, upon removal, strongly impair SNARE zippering [32]. These amino acids from the three SNAREs associate into layers of interacting residues in the center of the SNARE complex, as shown in Figure 4. The residue in the 0 layer, that is charged rather then hydrophobic as in other layers, provides an alternative classification for the SNARE proteins [33].

\subsubsection{SYNTAXIN}

Syntaxin-1a is a $33 \mathrm{kDa}$ membrane protein localized on the presynaptic membrane. It is called a t-SNARE (target SNARE) or the R-SNARE, because of the arginine residue in its zero layer. Its SNARE domain (189-262), also called the H3 domain, is a partial $\alpha$-helix [35], but gains further $\alpha$-helical character when in SNARE complex [27]. This segment also has the tendency to oligomerise, possibly leading to a homotetramer [36]. Another important domain is the $\mathrm{H}_{\mathrm{abc}}$, which is composed of three antiparallel $\alpha$-helices, $H_{a}(29-66), H_{b}(70-105)$ and $H_{c}(110-155)[27,37$, 38]. In order to interact with other two SNAREs to form the complex, only the SNARE domain is necessary [35]. In fact, a syntaxin construct possessing a point mutation that disrupts the interdomain $\mathrm{H} 3-\mathrm{H}_{\mathrm{abc}}$ contact exhibits more effective SNARE complex formation [39]. It is well documented that the $\mathrm{H}_{\mathrm{abc}}$ will interact with the SNARE domain $[23,40,41,42,43]$, thus diminishing its affinity to SNAP25 and synaptobrevin. This interaction results in syntaxin adopting a "closed conformation". The syntaxin configuration obtained from the crystal structure of syntaxin-Munc18 complex [43], as seen in Figure 5, is believed to represent the closed state. Syntaxin can also exist in an open state, with its two main domains further apart. The two states are thought to be in an equilibrium and 


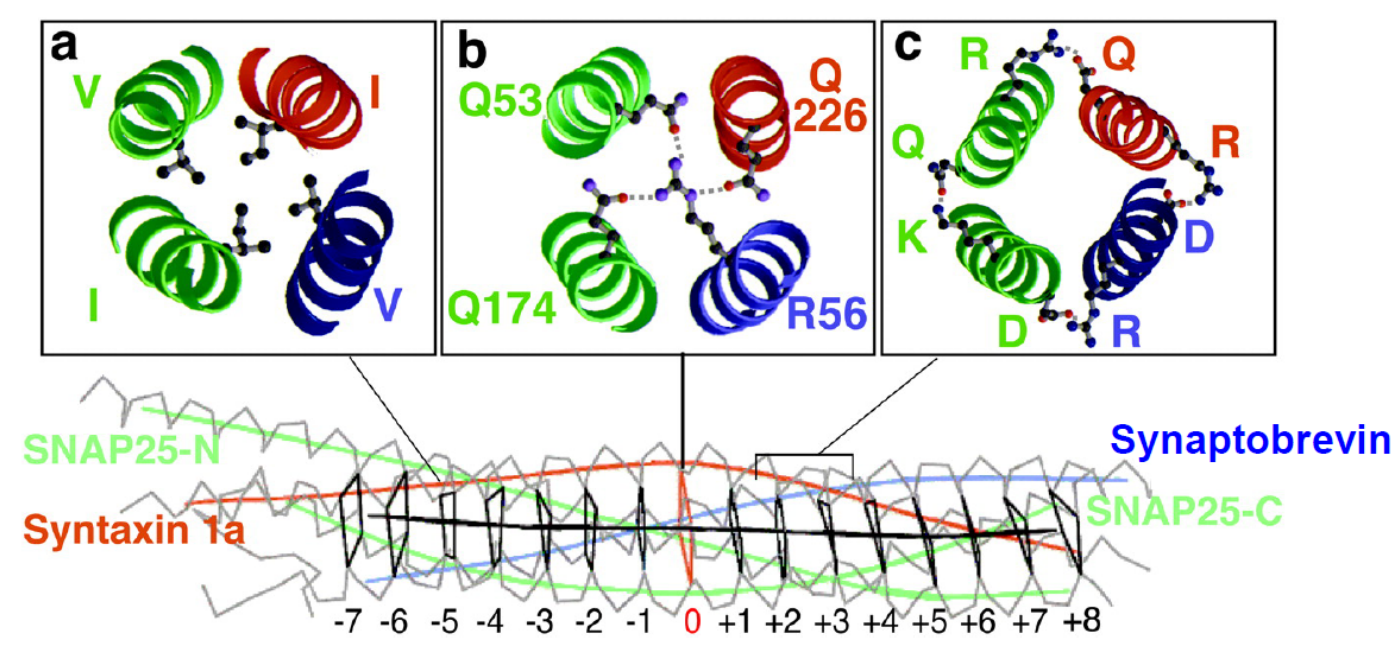

Figure 4. The hydrophobic layers of the SNARE complex. The central black polygons represent the highly conserved residues of three neuronal SNAREs. Most of the layers are composed of hydrophobic aminoacids (as in a). The central layer, called the 0-layer (b) is composed of charged residues and gives rise to alternative SNARE terminology: Q-SNAREs and R-SNAREs. Charged residues form hydrogen bonds outside the layer (c). Figure taken from reference 34. 
there are conflicting reports over which one is predominant $[40,41]$. The two domains are connected through an unstructured linker, that likely affects the openclosed equilibrium. Certain mutations in this linker will dramatically stabilize the open state and increase affinity to other two SNAREs [39].

Syntaxin also contains a short unstructured N-terminal segment (1-25) that is likely important for Munc18 binding. This segment is not required for binary syntaxin:Munc18 interactions [45], but is necessary for Munc18 to interact with the SNARE complex [46], highlighting at least two modes of binding of this protein to syntaxin. Syntaxin itself is anchored by a transmembrane domain (263-288) which may play a role in its oligomerization [47], as well as in interactions with other proteins [48].

\subsubsection{SNAP25}

Synaptosomal-associated protein 25 (SNAP-25) is a $24 \mathrm{kDa}$ Q-SNARE that provides two SNARE domains for the neuronal complex formation. It does not possess a transmembrane domain, instead, it is attached to the presynaptic membrane via a palmitoyl anchor [49]. It has two SNARE motifs, called SN1 (783) and SN2 (141-204), that are a largely unstructured segments, but will become $\alpha$-helical when interacting with syntaxin [50] or when in the SNARE complex [27]. SNAP25 can adopt a conformation where both its SNARE motifs come in contact and this state can be stabilized by other regulatory proteins [51].

\subsubsection{SYNAPTOBREVIN}

Synaptobrevin, also called VAMP2, is a $13 \mathrm{kDa}$ R-SNARE that provides one SNARE motif (30-85) to the SNARE complex. This protein is believed to be unstructured on the bilayer $[52,53]$, although a study in a detergent system showed 


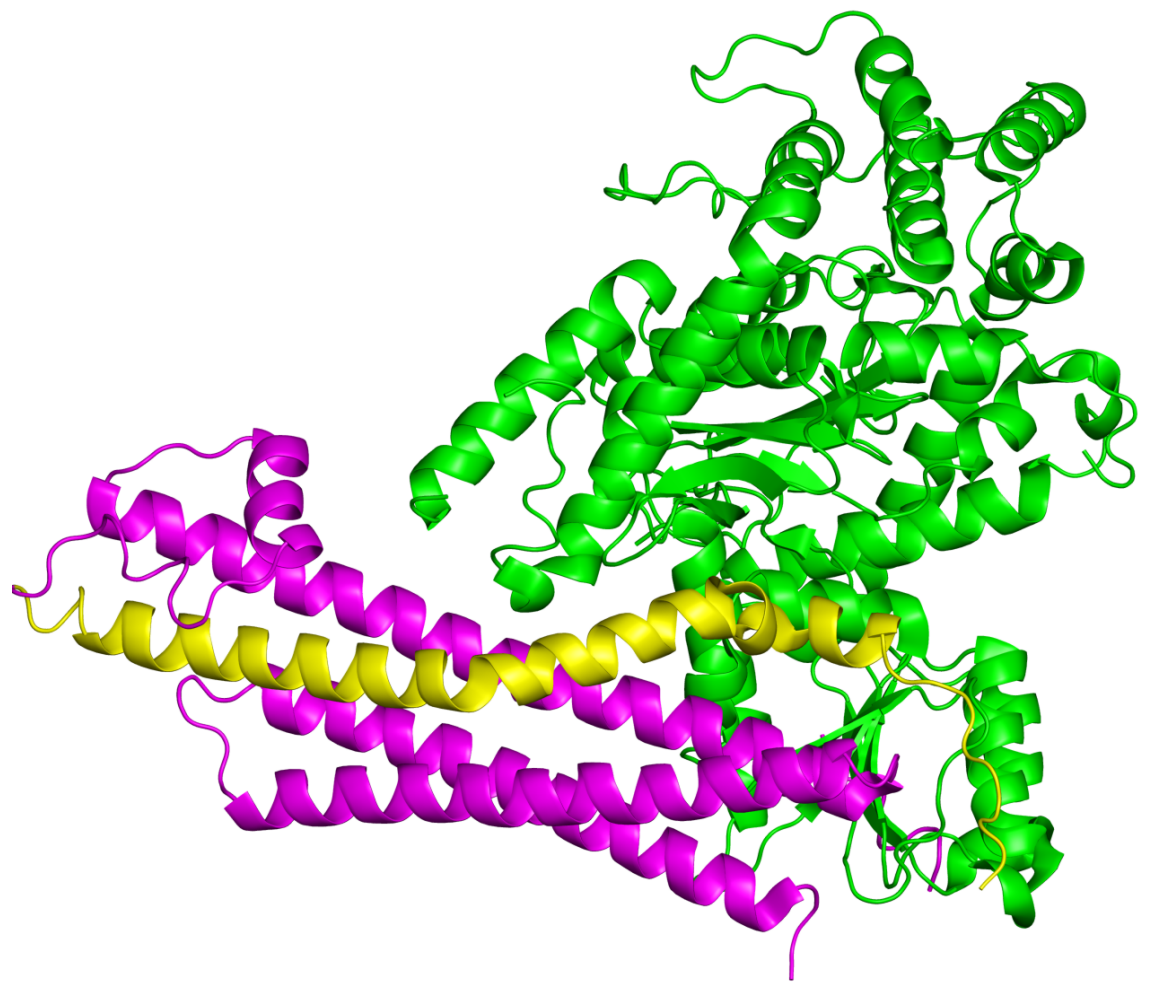

Figure 5. The crystal structure of syntaxin-Munc18 complex. Munc18 (green) binds simultaneously to the $\mathrm{H} 3$ (yellow) and $\mathrm{H}_{\mathrm{abc}}$ (magenta) domains of syntaxin. Syntaxin adopts a closed state with its two domains in direct contact. Figure made with PyMOL (DeLano Scientific LLC, Palo Alto, CA). PDB accession code: 3C98. 
propensity for greater helical structure [54]. As with the other two SNAREs, this segment will turn into a full $\alpha$-helix when in complex with other SNARE partners [27]. There are also reports of synaptobrevin contacting the lipid interface [55]. This is theorized to modulate its ability to form the SNARE complex, as its Nterminal segment is crucial to the initiation of SNARE zippering [56].

\subsection{BINARY SYNTAXIN:SNAP25 INTERACTION}

As SNAP-25 coexists with syntaxin on the presynaptic membrane, it is generally agreed that the two proteins can engage in important interactions that precede the SNARE complex formation and fusion. In fact, out of all the binary SNARE interactions (others being VAMP2-SNAP25 and syntaxin-VAMP2), this one results in the most stable complex [57]. This complex is often considered to be the "acceptor complex", as it is thought to represent the SNAREs before the arrival of the calcium trigger $[51,58,59]$.

Ideally, the 1:1 SNAP25-syntaxin complex, that would subsequently interact with synaptobrevin, providing a direct path to the SNARE complex formation. However, experiments in vitro indicate that a 2:1 complex with two syntaxins and one SNAP25 [60] is the dominant complex formed. This complex is believed to be an unproductive "off-shoot" pathway of the SNARE complex formation as the lipid fusion assay studies have shown that this complex drastically decreases the fusion efficiency [57].

\subsection{SYNTAXIN:MUNC18 INTERACTION}

Munc18 is one of the most important regulatory proteins of neuronal fusion. In vivo 
study of neurons with a Munc18 knock-out revealed inhibition of fusion at early states of vesicle priming [61]. This protein was initially discovered on the basis of its affinity for syntaxin [62]. The crystal structure of syntaxin-Munc18 complex [43], shown in Figure 5, presents what is often considered the main role of this protein: arresting syntaxin in its closed state. This makes the SNARE motif of syntaxin less accessible for interaction with synaptobrevin and SNAP25 [57, 63]. The mutations in the linker region of syntaxin, that stabilize the open state of syntaxin, also inhibit binding of Munc18 [39].

Stabilizing the closed state of syntaxin by Munc18 should seemingly be a purely negative factor that decreases the fusion efficiency. However in vivo studies revealed a stimulatory effect of Munc18 [61, 64], even identifying it as the most upstream essential protein in neuronal fusion [61]. This means the effect of this protein on fusion may extend past the binary syntaxin-Munc18 interactions. Indeed, a second mode of interaction was found, where Munc18 remains in contact with the fully formed SNARE complex through the short N-terminal segment of syntaxin $[65,66]$.

\subsection{MUNC13 AND ITS MAIN DOMAIN MUN}

Munc13-1 is a crucial element of the neuronal fusion process. Its removal impairs this process at the early stage of vesicle priming [67-69]. It is a large $200 \mathrm{kDa}$ protein possessing multiple domains. These domains seem to have disparate roles, such as the $\mathrm{C} 1$ binding to diacylglycerol [70], $\mathrm{C} 2 \mathrm{~B}$ being sensitive to phospholipids in a calcium-dependent manner [71] and the MUN domain that is reported to act directly on the SNARE proteins/SNARE complex [72]. So far, it has proved 
impossible to purify the entire Munc13, but studies have been performed with its partial construct $[72,73]$.

Among the domains of Munc13, the MUN domain was revealed to be crucial in SNARE interaction $[51,72]$. MUN is reported to be capable of opening syntaxin, when this protein is in complex with Munc18. This effect on syntaxin leads to improved kinetics of SNARE zippering and of lipid fusion [73]. In addition, an in vivo study revealed that the mutations that result in constitutive open syntaxin will rescue the neurotransmitter release of the $C$.elegans Munc13 null mutant, further supporting the idea of the MUN domain as the opening effector for syntaxin [74].

\subsection{SPECIFIC AIMS}

The purpose behind the work presented here was fourfold. The first aim was to elucidate the tertiary structure of syntaxin against previous conflicting reports and study its change caused by Munc18 binding. This work is described in Chapter 3 of this thesis. The second objective was to see if the MUN domain of Munc13 can alter the structure of syntaxin in a syntaxin-Munc18 complex. No effect of MUN domain on syntaxin was observed, which points to a role of Munc13 in directing other elements of the fusion system If this effect was confirmed, then additional experiments would have been pursued to try to understand the mechanism of MUN action. The third aim focused on interactions between syntaxin and SNAP25. These two proteins can form at least two different, vesicle-fusion relevant complexes, and therefore it was important to analyze their structure and see under what conditions do each of these complexes become more stable and predominant. The last aim was to analyze the structure of synaptobrevin in micelle, bicelle and lipid environments. 
As discussed in this thesis, synaptobrevin exhibited a very different behaviour on lipid bilayer surfaces than in solution, observations that could be explained by transient interactions of SNARE heptad repeats with the bilayer interface. The work presented in Chapter 4 demonstrates with a combination of EPR and NMR spectroscopy that such interactions may take place.

All the proteins of neuronal fusion have already been analyzed to some detail, but many studies either yielded contrary results or were not yet confirmed by complementary studies in the literature. This work therefore aimed at confirming some of the published hypotheses. More importantly however, this EPR study tried to provide a detailed biophysical insight on the interactions between the SNARE proteins, as the current understanding of the neuronal fusion is arguably dominated by functional assay studies, and is lacking in information on a biomolecular level. It was also attempted to combine some, if not all the above-mentioned investigations into a single structural study that would yield a uniform conclusion addressing the question of the mechanism of the SNARE-mediated neuronal fusion. 
Chapter 2: Electron Paramagnetic Resonance $(\mathrm{EPR})$ 


\subsection{FUNDAMENTAL THEORY}

EPR spectroscopy is a well established biophysical technique that allows for accurate analysis of the dynamics of a spin label, which can be site-specifically introduced into a protein. This gives valuable information about the protein backbone dynamics and any tertiary contact that a protein segment may have. Transitions between different conformational states can also be detected. The measurement may be done in room temperature, in a low salt buffer and without the presence of any detergents or osmolytes. This in turn provides an opportunity for accurate analysis of subtle conformational transitions, which may not be possible when a molecule is placed in solution containing more aggressive solutes like osmolytes, that are necessary for protein crystallization.

The heart of the EPR lies in the ability of the unpaired electron, when subjected to a magnetic field, to adopt one of two separate energy states. This phenomena, called a Zeeman splitting, is represented by a electronic Hamiltonian:

$$
H_{z}=\mathrm{g} \beta \mathrm{S}_{\mathrm{z}} \mathrm{H}_{0}
$$

where $g$ is the "g-factor", $\beta$ is the Bohr magneton, $S_{z}$ is a spin angular momentum (when the spin is aligned along the $\mathrm{z}$ axis) and $\mathrm{H}_{0}$ is the applied magnetic field. The eigenvalues of $S_{z}$ are $-1 / 2$ and $+1 / 2$, which are the two possible values of the spin angular momentum of an electron. Therefore, the spin energies are:

$$
\mathrm{E}= \pm \frac{1}{2} g \beta \mathrm{H}_{0}
$$

and the energy difference between the two states is:

$$
\Delta \mathrm{E}=\mathrm{g} \beta \mathrm{H}_{0}
$$

so that the energy difference between those two states is linear with the field $\mathrm{H}_{0}$ (Figure 7) 


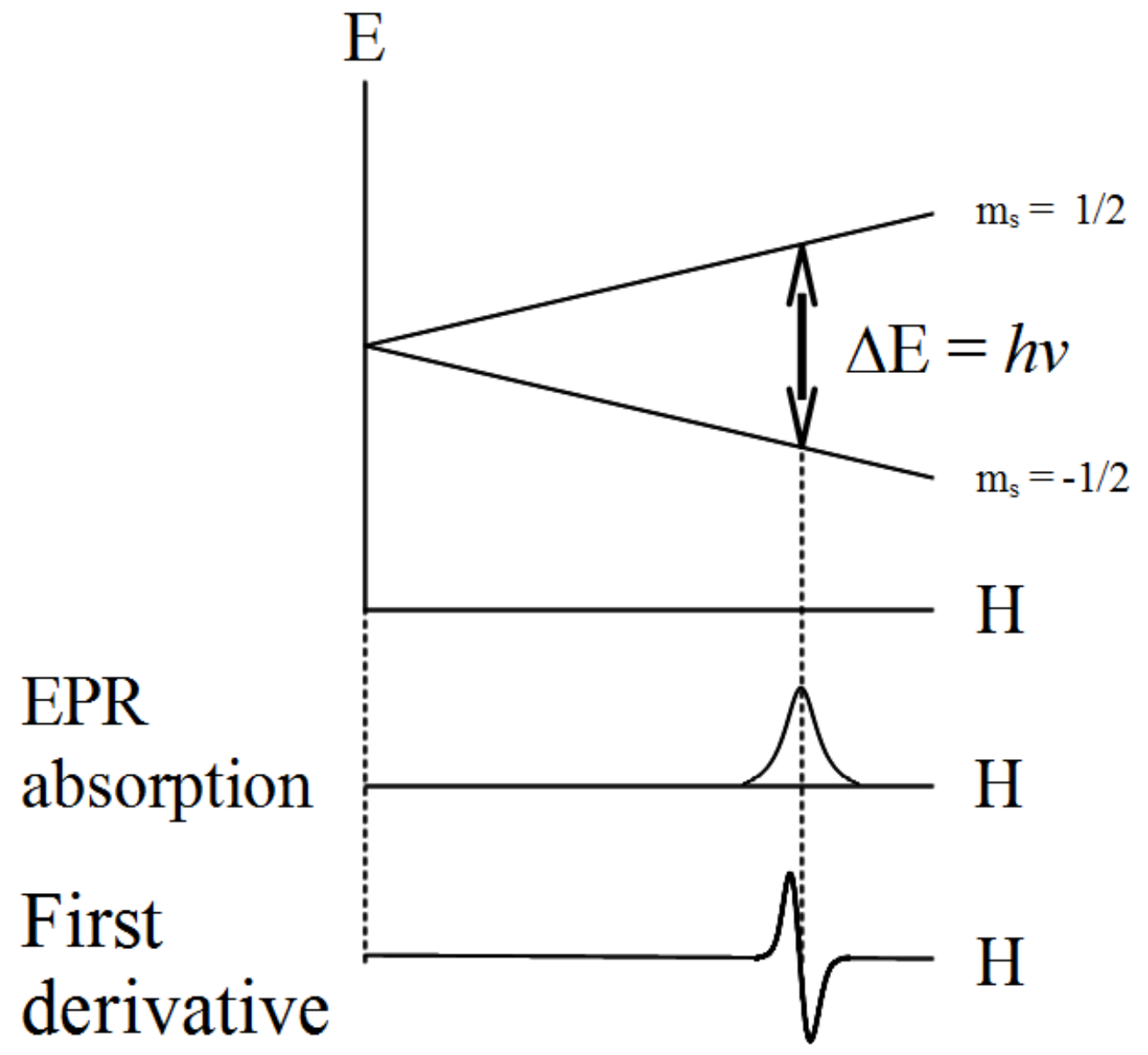

Figure 7. Energy level splitting due to Zeeman interaction.

In the presence of the external magnetic field $\mathrm{H}$, the unpaired electron can adopt two distinct energy levels. This allows it to absorb an energy quanta $\Delta \mathrm{E}$ to transition from the lower to the higher energy state. 


\subsection{CONTINUOUS WAVE EPR (CW EPR)}

The CW EPR technique relies on analysis of the microwave absorption by a sample as a function of an applied magnetic field $\mathrm{B}_{0}$. There are several phenomena that affect the shape of the absorption spectrum. First of all, the previously mentioned gfactor is a parameter that indicates the position of the absorption spectrum in the field of frequency dimension, according to the equation:

$$
\mathrm{g}=\mathrm{h} \mathrm{v} / \beta \mathrm{H}_{0}
$$

This value is unique for different paramagnetic species and is also affected by the rate of their molecular motion. The g-factor is a tensor, which can be averaged by sufficiently high rapid tumbling to the isotropic value of $\mathrm{g}_{0}$ :

$$
\mathrm{g}_{0}=1 / 3\left(\mathrm{~g}_{\mathrm{xx}}+\mathrm{g}_{\mathrm{yy}}+\mathrm{g}_{\mathrm{zz}}\right)
$$

approaching the value for the free electron: $g=2.00232$. If the overall tumbling is not fast enough $\left(<10^{9} \mathrm{~Hz}\right)$, then the three principal values will be resolved in the spectrum. For the nitroxide spin label on a protein surface, these tensor values are $g_{x x}=2.009, g_{y y}=2.006, g_{z z}=2.002[76]$. The way the different principal values of the g-factor shift the center of the spectrum is shown in Figure 8.

Another factor that has a significant impact on the shape of absorption spectrum comes from the interaction of a magnetic moment of an electron spin with a nuclear magnetic moment of a local nucleus. This phenomena, termed the hyperfine interaction, manifests itself in the nitroxide spin labels, where the unpaired electron, located mostly on the $2 p \pi$ orbital of the nitrogen atom, interacts with the nucleus of the nitrogen, which has three spin quantum values. From this 

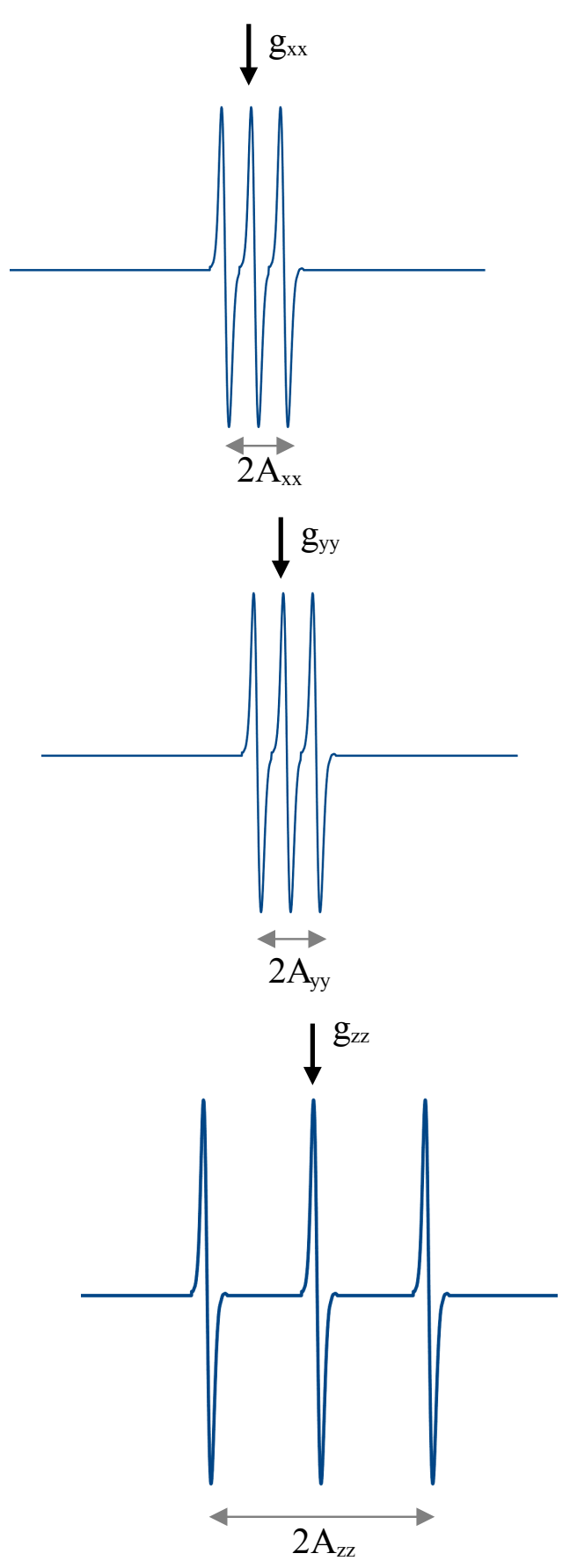

Figure 8. The orientational effect on the g-factor and the hyperfine tensor.

The spectra resulting from the $\mathrm{x}$-axis (top), $\mathrm{y}$-axis (middle) or z-axis (bottom) of the nitroxide reference frame (shown in Figure 10) aligning with the magnetic field. Vertical arrows indicate centers of the spectra in the field dimension, shifted due to different value of g-factor. Length of horizontal arrows show the value of the hyperfine tensor, which can change dramatically when the nitroxide is in the z-axis alignment. 
interaction the EPR spectrum splits into three signals, which corresponds to the electron interacting with the three nuclear states. This provides an additional component to the Hamiltonian:

$$
H=g \beta \mathrm{S}_{\mathrm{z}} \mathrm{H}_{0}+\mathrm{S} \cdot \mathrm{A} \cdot \mathrm{I}
$$

where $\mathrm{S}$ is the electron spin angular momentum, $\mathrm{I}$ is the nuclear spin angular momentum, and $\mathrm{A}$ is the hyperfine tensor. The hyperfine tensor represents the electron-nuclear interaction, which is anisotropic. This in turn has a very significant influence on the EPR spectra, which will ultimately allow one to monitor the dynamics of the spin label. As presented in the Figure 9, the magnitude of the hyperfine tensor can be seen as the distance between the two absorption signals. This value however, due to A being a tensor, is dependent upon orientation of the nitroxide magnetic frame to the field (shown in Figure 8, with the magnetic frame described in Figure 10). When the field is placed along the x-axis or y-axis, the hyperfine tensor values, determined as: Axx $=6.2$, Ayy $=5.9$ for the nitroxide [76], are very similar. There is however a major difference if the field lies along the z-axis, since the $A_{z z}$ rises to 37 . This difference can be easily observed when running the $\mathrm{CW}$ experiment on a protein crystal, simply by changing the orientation of a crystal. And if the crystal was to be grounded into a powder, than the spectrum would represent all three orientations of the spin.

In solution, the rotational diffusion can average the hyperfine and g-factor tensor values. In order for a complete averaging to take place, the motion needs to be fast enough in the CW EPR timeframe. A parameter that describes the rotation of the molecules is the rotational correlation time $\left(\tau_{\mathrm{r}}\right)$ : Figure 11 shows that the slower the movement, the less averaging of the A tensor - to the point of a "powder 




Figure 9. Splitting of the nitroxide energy levels due to the hyperfine interaction.

The hyperfine interaction of the electron with the nitrogen nucleus results in additional splitting. The value of the hyperfine tensor can be directly measured in Gauss on the $\mathrm{x}$-axis as the separation A between the absorption peaks. Also shown is the first derivative of the absorption profile. Figure made based on reference 100 . 


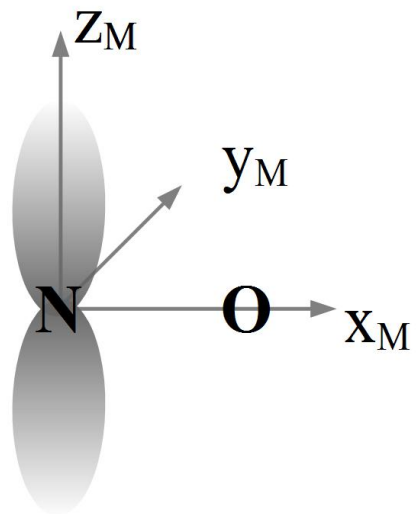

Figure 10. The magnetic frame of the nitroxide. The external magnetic field vector is aligned parallel to the p-orbital of the nitrogen, therefore also parallel to the $\mathrm{z}$-axis of the reference frame. 

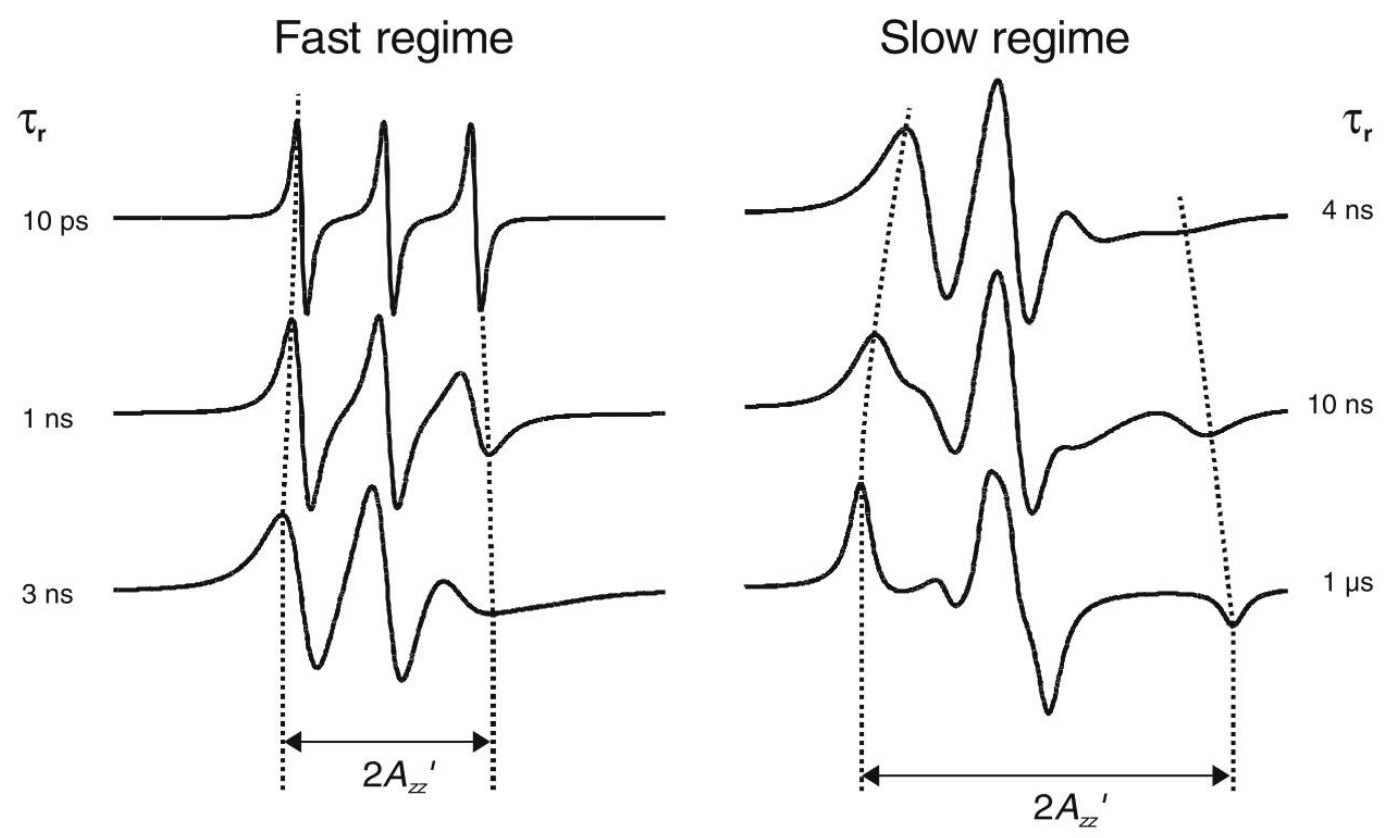

Figure 11. CW lineshape changes due to the different correlation times of the nitroxide.

Faster motion of the nitroxide results in more averaging of the $g$ and A tensors that has a clear impact on the shape of the spectrum. Figure taken from reference 77. 
crystal" where there is no averaging. This means, that by the analysis of the CW spectrum, one can obtain the correlation time of the rotation of the spin label. That in turn will yield useful information regarding the part of the protein where the spin label is attached, as the motion of the protein backbone will add to the motion of the label. An ordered $\alpha$-helix will exhibit less backbone motion than the random coil, and such difference will be reflected in the correlation time. Also, a tertiary contact will significantly reduce the motion of a spin label protein surrounding, again, impacting the $\tau_{\mathrm{r}}$ directly. Therefore, the effect of protein motion on a EPR spectrum is sensitive enough to distinguish different secondary structures, as presented in a Figure 12.

Analysis of the EPR lineshapes can yield semiquantitative parameters that describe the relative mobility of the nitroxide. These parameters are a function of the correlation time $\tau_{\mathrm{r}}$ and the order potential S (described in the next paragraph), and can be obtained easily from the lineshapes without the need for complex fitting of the spectra. One of these parameters is the peak-to-peak amplitude of the central line, shown in Figure 13. This parameter increases in value, as the correlation time gets lower and spectral lines become sharper, and is easy to measure accurately owing to the central line being of the highest amplitude in the EPR spectrum, and therefore being the least susceptible to noise. Another parameter is the inverse linewidth of the central line, $\Delta \mathrm{H}_{\mathrm{pp}}$ or $\delta^{-1}$, which also measures the mobility of the spin label (Figure 13). The inverse linewidth can also be normalized against the linewidths of the least and most mobile EPR spectra that were reported in the literature. Such parameter, termed the scaled mobility or $\mathrm{m}_{\mathrm{s}}[78,99)$ is defined as:

$$
m_{s}=\frac{\delta^{-1}-\delta_{i}^{-1}}{\delta_{m}^{-1}-\delta_{i}^{-1}}
$$




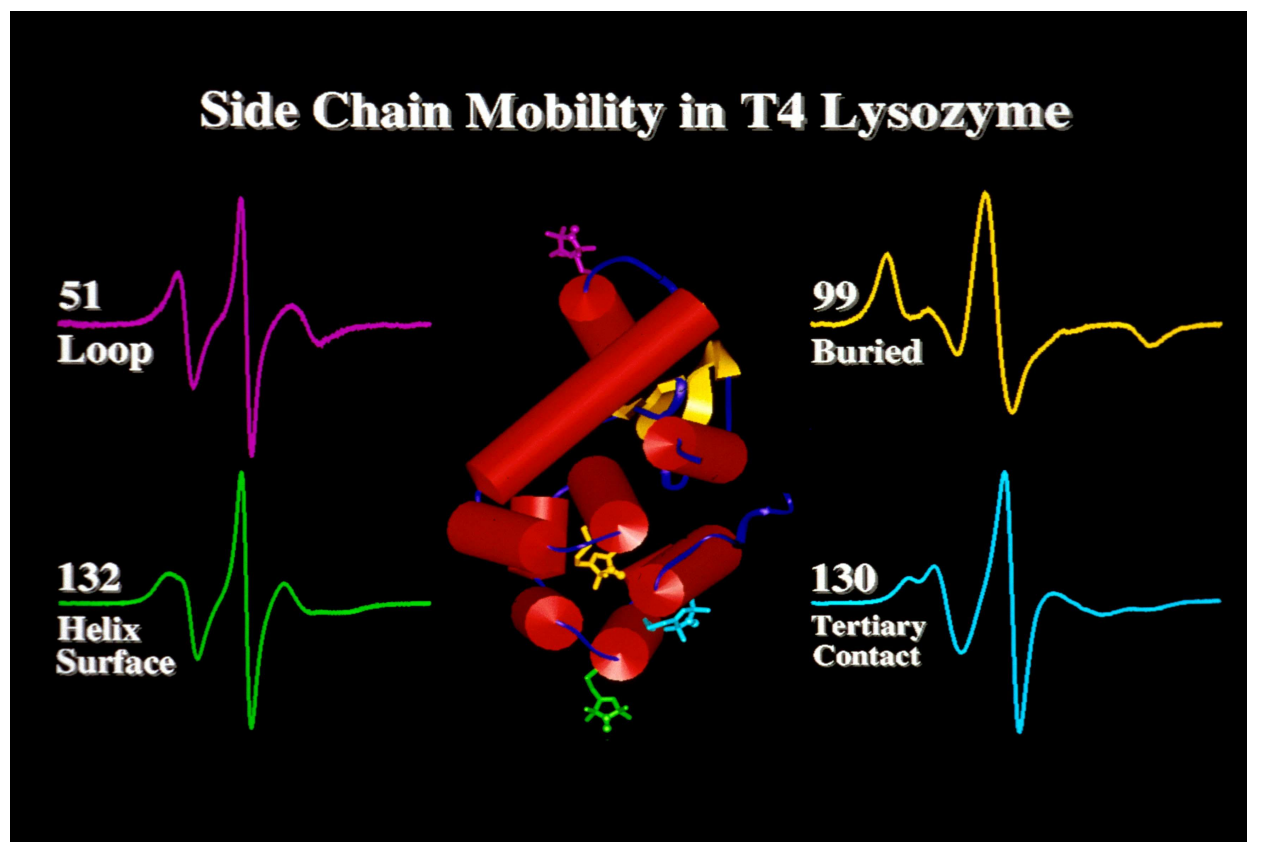

Figure 12. Illustration of CW EPR spectra taken in different protein structural environments

The CW study on the T4 lysozyme revealed that each protein secondary structure gives a unique EPR spectrum. This is due to the fact that the particular nitroxide used in the study - MTSL, is very sensitive to the protein backbone motion. Other nitroxide molecules that would possess higher degrees of sidechain freedom, would be less sensitive to the differences in protein secondary structure. Figure taken from reference 80 . 


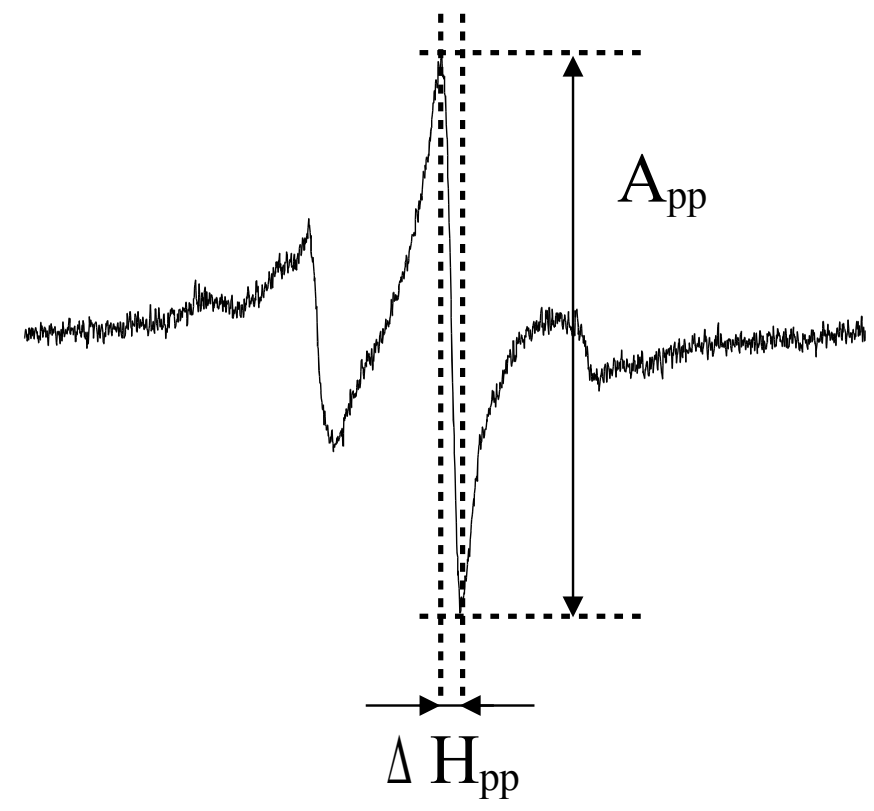

Figure 13. Peak-peak amplitude and the central line width of the $\mathrm{CW}$ spectrum. Both parameters can measure the relative mobility of the nitroxide, and can be obtained directly from the spectrum. 
where $\delta_{i}^{-1}$ and $\delta_{m}^{-1}$ are inverse linewidths obtained from least and most mobile spectra, respectively.

The correlation time of the spin label can be estimated by using the MOMD model for motional averaging. The Microscopic Order Macroscopic Disorder (MOMD) model was developed by Jack Freed and coworkers [79] and may be used to simulate EPR lineshapes based upon models for molecular motion. This method assumes an additional reference frame with three principal axes $x_{R}, y_{R}, z_{R}$ that define a rotational diffusion tensor $\mathrm{R}$, which dictates the rate of motion of the nitroxide. The MOMD routine also restricts the diffusion of the spin label to the surface of a cone with the angle $\theta$ about the z-axis of the rotational diffusion frame. This limitation of the spin label diffusion is defined by the ordering potential:

$$
\mathrm{S}=1 / 2\left(3\left(\cos ^{2} \theta\right)-1\right)
$$

where $\theta$ is the angle between the axis of the cone and the $\mathrm{Z}_{\mathrm{R}}$ axis of the nitroxide rotational diffusion frame. This restriction defined the microscopic order that is imposed on the spin labeled protein molecule. The protein backbone however is free to diffuse, with the principle axis $x_{R}, y_{R}, z_{R}$ not being restricted against the magnetic frame. This results in macroscopic disorder in the system composed of multiple spin labeled molecules.

The MOMD approach is implemented in the program Multicomponent by Christian Altenbach, that allows for computational approach for simultaneous fitting of several parameters to reproduce the EPR spectrum. Of particular importance is its ability to fit several spectral components that result from the nitroxide exhibiting at least two separate motions. Final result of the MOMD spectral fitting will provide the populations of each components, which is a 
valuable information on the protein system being studied, particularly if those populations shift after a change in experimental conditions. Also, successfully reproduced spectral fits will reveal the value of the rotation diffusion tensor $\mathrm{R}$, and therefore the correlation time $\tau_{\mathrm{r}}$ via the equation:

$$
\tau_{\mathrm{r}}=1 / \mathrm{R}_{\mathrm{av}}
$$

where $R_{a v}$ is the $R$ tensor that is a geometrical average of its principal values $\mathrm{R}_{\mathrm{x}}, \mathrm{R}_{\mathrm{y}}, \mathrm{R}_{\mathrm{z}}$.

\subsection{DOUBLE ELECTRON-ELECTRON RESONANCE (DEER)}

The DEER is a pulse EPR technique that allows distances to be measured between two labels that fall within the $1.5-8 \mathrm{~nm}$ range [81, 82]. This technique can be applied to soluble [83] and membrane proteins [84], as well as to peptides [85] and nucleic acids [86].

The distance information is obtained by extracting the dipolar function between unpaired electrons of two spin labels. The Hamiltonian for the DEER experiment can be written as:

$$
H_{\text {dipolar }}=\left(\mu_{0} / 2 \mathrm{l} r_{A B}^{3} \mathrm{~g}_{\mathrm{A}} \mathrm{g}_{\mathrm{B}} \beta^{2} \mathrm{~S}_{\mathrm{A}, \mathrm{Z}} \mathrm{S}_{\mathrm{B}, \mathrm{Z}}\left(3 \cos \theta^{2}-1\right)\right.
$$

where $\theta$ is the orientation of the interspin vector relative to the external magnetic field, $\mu_{0}$ is the permeability of a vacuum, $\mathrm{h}$ is the Planck constant, $\mathrm{g}_{\mathrm{A}}$ and $\mathrm{g}_{\mathrm{B}}$ represent the isotropic g-values of the A and B spins which are coupled, $S_{A}$ and $S_{B}$ are the spin angular momentum operators of the A and B spins and $r$ is the interspin distance between them.

In order to describe the pulsed EPR, it is important to visualize the behavior of spins in a coordinate system. In the most common axis convention system, the 
magnetic field $B_{0}$ is parallel to the $z$ axis, the microwave magnetic field $B_{1}$ is parallel to the $\mathrm{x}$ axis, and the $\mathrm{y}$ axis is orthogonal to the $\mathrm{x}$ and $\mathrm{z}$ axes (in accordance with the magnetic frame in Figure 10). When the spin experiences a magnetic field, a torque is exerted on it, causing precession of its magnetic moment about the magnetic field. The precession occurs with the angular frequency of $\omega_{\mathrm{L}}$, called the Larmor frequency, which is related to the magnetic field by:

$$
\omega_{\mathrm{L}}=-\gamma \mathrm{B}_{0}
$$

where $\gamma$ is the gyromagnetic ratio. An electron spin will align either parallel or antiparallel along the $\mathrm{B}_{0}$, which corresponds to the lower and higher energy state, respectively. According to the boltzmann distribution, there will be a surplus of spins in the parallel alignment which will precess along the $\mathrm{z}$ axis. The spins however will be rotating independently, which means that, when considering a large enough assemble, they will cancel out in the x-y plane. Only their z-axis component will be present, and it will give rise to a stationary magnetization, termed $\mathrm{M}_{0}$ (Figure $14 \mathrm{~B}$ ). The stationary magnetization can be tipped off the $\mathrm{z}$-axis with the microwave magnetic field $\mathrm{B}_{1}$, which will cause the magnetization to precess along the $\mathrm{x}$-axis. The angle by which the magnetization is rotated, commonly called the tip angle, is equal to:

$$
\alpha=-\gamma B_{1} t_{p}
$$

where $t_{p}$ is the timing of the $B_{1}$ magnetic field pulse. As shown in Figure $14 \mathrm{C}$, using a $B_{1}$ pulse with a proper timing, the magnetization can be tipped with a $\pi / 2$ angle, so that it will be aligned along the $y$-axis. Another type of a pulse is a $\pi$ pulse that, having twice the length of a $\pi / 2$ pulse, tips the magnetization along the $-\mathrm{z}$ axis. The actual signal recorded during the pulse experiment originates from an FID (Free Induction Decay). FID are the electrical currents, that are being generated by 
A

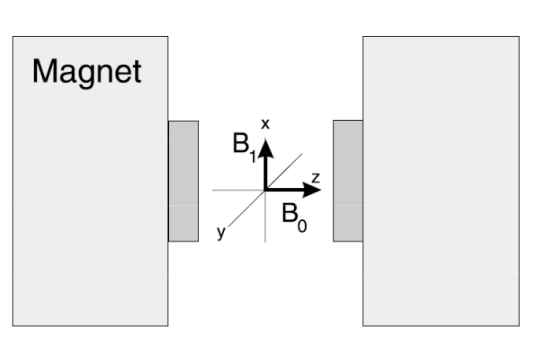

C
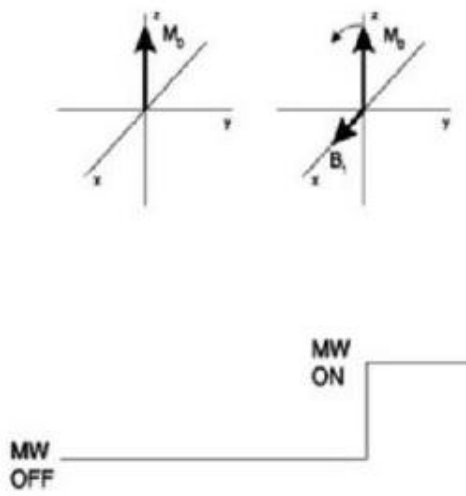
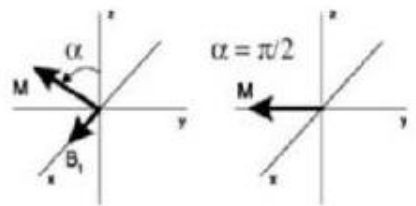

B

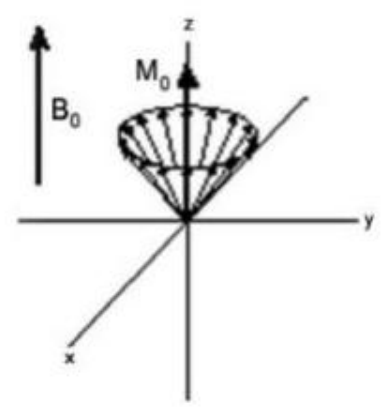

WW

N

Figure 14. A The magnetic reference frame. B. Magnetization vector $M_{0}$ as a sum of z-components of individual magnetic moments. C. Tipping of the magnetization vector $\mathrm{M}_{0}$ in the $\mathrm{x}-\mathrm{y}$ plane due to application of a microwave pulse. Figure modified from reference 87 . 
the spins, while they rotate along the z-axis (the detection lies on the $x-y$ plane). Since the individual spins experience different magnetic fields, they also rotate at slightly different angular frequencies. Because of that after the pulse the spins start to dephase in the $x-y$ plane. This in turn leads to the canceling out of their signal in the $x-y$ plane and the decrease of transverse magnetization (Figure 15 A). This however can be reversed with the $\pi$ pulse, which will have a refocusing effect on the spins, rescuing their coherence in the $x-y$ plane (Figure 15 B). The $\pi$ pulse results in an echo, which is an electromagnetic signal detected in the $\mathrm{x}-\mathrm{y}$ plane, that comes from the non-zero transverse magnetization that precesses about the z-axis.

The spin phase coherence in the xy-plane, that is detected in the DEER experiment, can be lost via the spin-spin relaxation unless the experiment is performed in sufficiently low temperature. The spin-spin relaxation is the mechanism through which the perpendicular component of the magnetization decays to zero with the time $\mathrm{T}_{2}[88]$ defined as:

$$
\frac{d M_{x y}}{d t}=\frac{-M_{x y}}{T_{2}}
$$

In room temperature the $T_{2}$ for the nitroxide is on the order of $30 \mathrm{~ns}$, which is too short, and would result in total eradication of the xy-plane spin coherence. However, dropping the temperature to around $50-80$ Kelvin increases the $\mathrm{T}_{2}$ sufficiently to make the DEER experiment possible [89]. It is important to note, that the $T_{1}$ time of the spin-lattice relaxation (described in section 2.3) increases as well, and this diminishes the number of repeats of the pulse sequence experiments that can be performed on the sample in a specific amount of time. Nevertheless the longer $\mathrm{T}_{2}$ allows for greater degree of the $\mathrm{x}-\mathrm{y}$ plane coherence rescue, even after a 
A

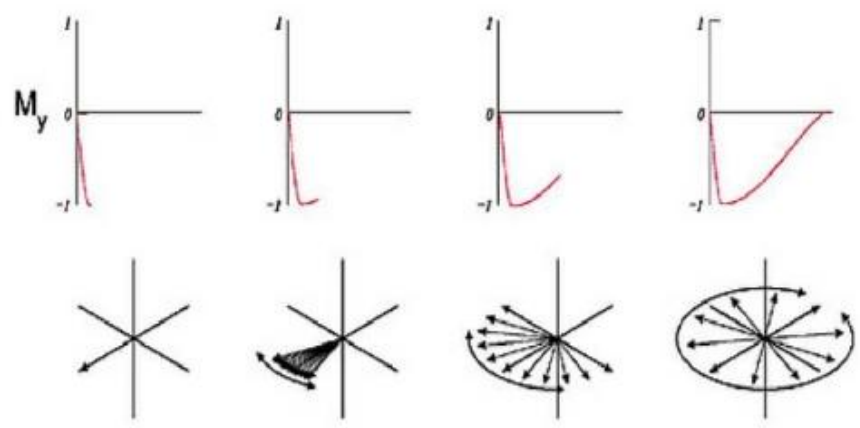

B

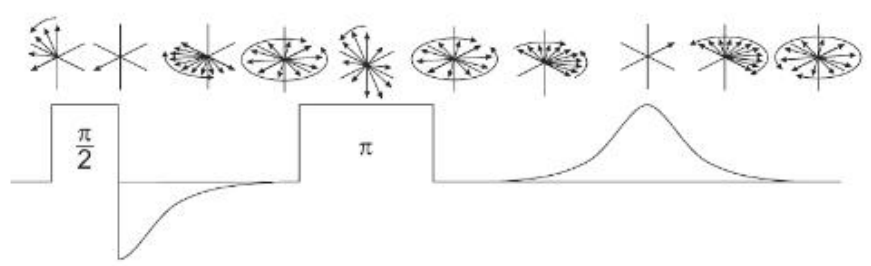

C

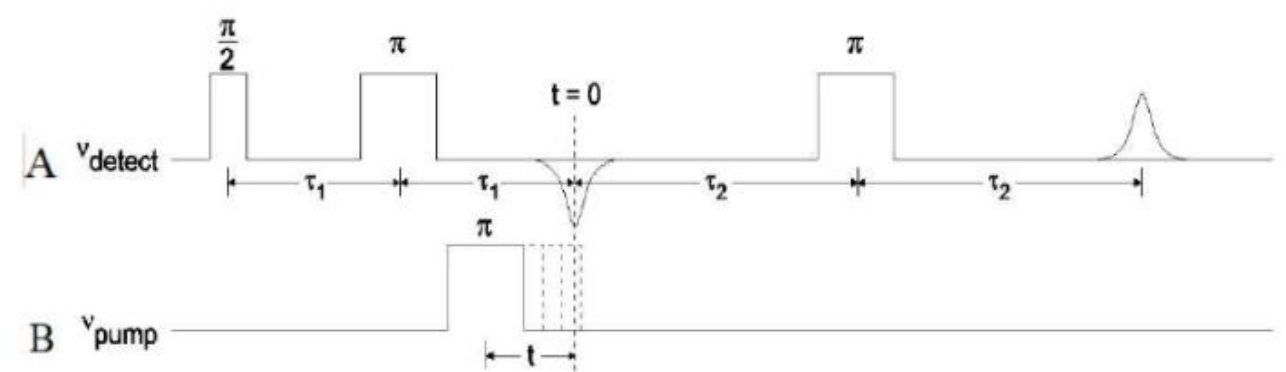

Figure 15. A. Echo intensity, being a sum of electromagnetic signals coming from spins coherently precessing in the $\mathrm{x}-\mathrm{y}$ plane, will decay as the spins lose coherence with time. B. Additional $180^{\circ}$ pulse reverses the order of spin precession, rescuing their coherence. This allows to create the echo, from the FID that was generated with the initial $90^{\circ}$ pulse. C. The Pulse sequence used in the DEER experiment. Figures taken from reference 87. 
longer delay between the refocusing $\pi$ pulse, which becomes particularly relevant for the determination of the longer distances. Aside from the $\mathrm{T}_{2}$, other mechanisms can also affect the xy-plane coherence. Effects such as spin, spectral and instantaneous diffusion add to the effect of $\mathrm{T}_{2}$ [90], resulting in an empirical parameter phase-memory time $\left(\mathrm{T}_{\mathrm{m}}\right)$ that can provide valuable information on the protein system. This parameter can be measured with a specific pulse experiment in addition to the DEER experiment.

The DEER technique relies on careful manipulation of two groups of spins: A spins at the "observe" position, and the B spins at the "pump" position. These two positions are selected from different locations on the absorption spectrum of the nitroxide. An example of a spin group selection is shown in figure 16 for the DEER experiment performed at the Q-band microwave frequency. The two groups of nitroxides can be selectively excited by a pulse sequence, an example of which is shown schematically on Figure 15 C. During the experiment, after the first $\pi$ pulse that influences the A spins, there is a $\pi$ pulse on the B spins. This pulse will change the dipolar contribution from the B spins that is felt by the A spins. This way, there will be a phase shift which will influence the refocusing of the A spins. The final $\pi$ pulse on those spins will yield an echo, that will be recorded as a result of the experiment. This experiment is then repeated by changing the time of the pump pulse influencing the B spins. The final result will be the echo intensity plotted as a function of pump pulse timing, which will oscillate at the dipolar frequency. This signal is [82]:

$$
\mathrm{V}\left(\mathrm{t}_{\mathrm{p}}\right)=\cos \left(\omega_{\mathrm{AB}} \mathrm{t}_{\mathrm{p}}\right)
$$

where $t_{p}$ is the time when the pump pulse is applied and $\omega_{\mathrm{AB}}$ is the angular 


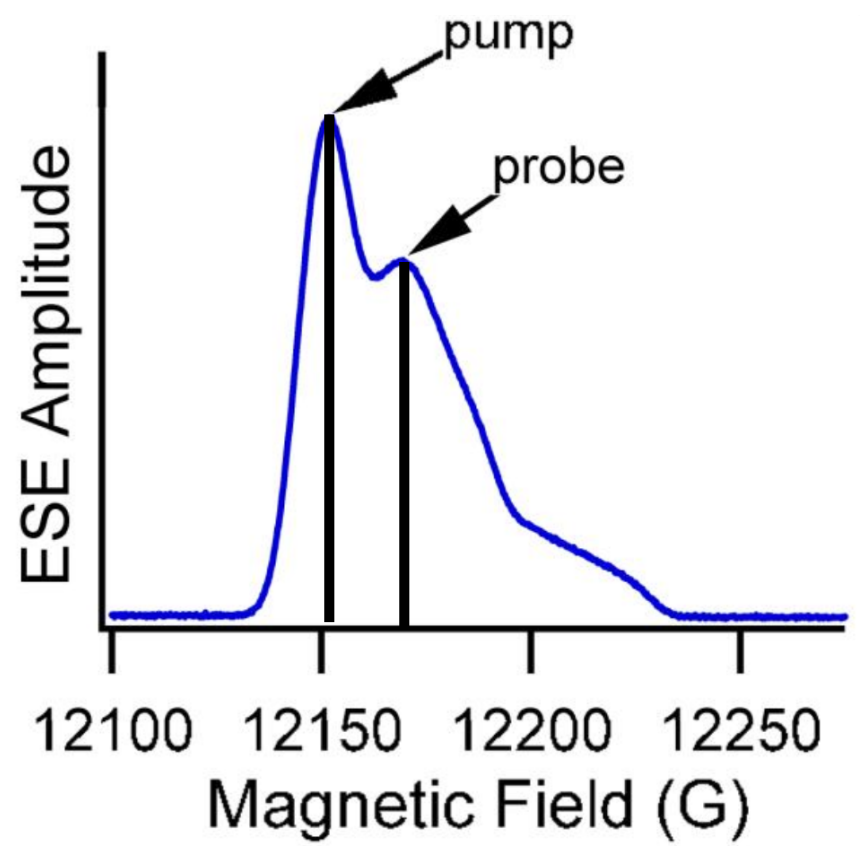

Figure 16. Echo detected field sweep spectrum.

The pump and observe (probe) positions for the spectrum taken at $35 \mathrm{GHz}$ (Qband). Figure adopted from reference 91. 
frequency of the dipolar interaction. The angular frequency $\omega_{\mathrm{AB}}$ is equal to:

$$
\omega_{A B}=\left(\mu_{0} / 2 \mathrm{~h} r_{A B}^{3} \mathrm{~g}_{\mathrm{A}} \mathrm{g}_{\mathrm{B}} \beta^{2}\left(3 \cos \theta^{2}-1\right)\right.
$$

Owing to its $\mathrm{r}^{-3}$ distance dependence, analyzing the oscillations will allow for the distance between the spins to be calculated.

The analysis of the DEER data is complicated by the fact that observable signal will result from intra as well as intermolecular spin interactions (81):

$$
\mathrm{V}(\mathrm{t})=\mathrm{V}_{\text {intra }}(\mathrm{t}) \mathrm{V}_{\text {inter }}(\mathrm{t})
$$

The intermolecular interactions will involve the interactions of the spin on one protein molecule with all the nearby spins in the solutions. The intermolecular signal will take a form of an exponential decay dependent on the spin concentration (C) and on the fraction of the spins excited by the pump pulse $\left(\lambda_{B}\right)$ :

$$
\mathrm{V}_{\text {inter }}(\mathrm{t})=\exp \left(-\mathrm{kC} \lambda_{\mathrm{B}} \mathrm{t}\right)
$$

where $\mathrm{k}$ is:

$$
\mathrm{k}=16 \pi^{3} \mathrm{~g}_{\mathrm{A}} \mathrm{g}_{\mathrm{B}} \beta^{2} /\left(9 \cdot 3^{0.5} \mathrm{~h}\right)
$$

The intermolecular signal $V_{\text {inter }}$ needs to be divided out from the final echo modulation signal in order to obtain the intramolecular signal from which the dipolar function can be extracted. This process is shown in Figure 17. This Figure also shows how modulation depth is estimated. This parameter informs on the relative percentage of spins participating in dipolar interaction, and can be used, alongside the distance estimate, to gain information on the spin labeled system.

The dipolar evolution spectrum obtained from a background-substracted echo modulation can be Fourier-transformed to obtain frequency-domain DEER spectrum from which the distance information can be directly obtained. Such 

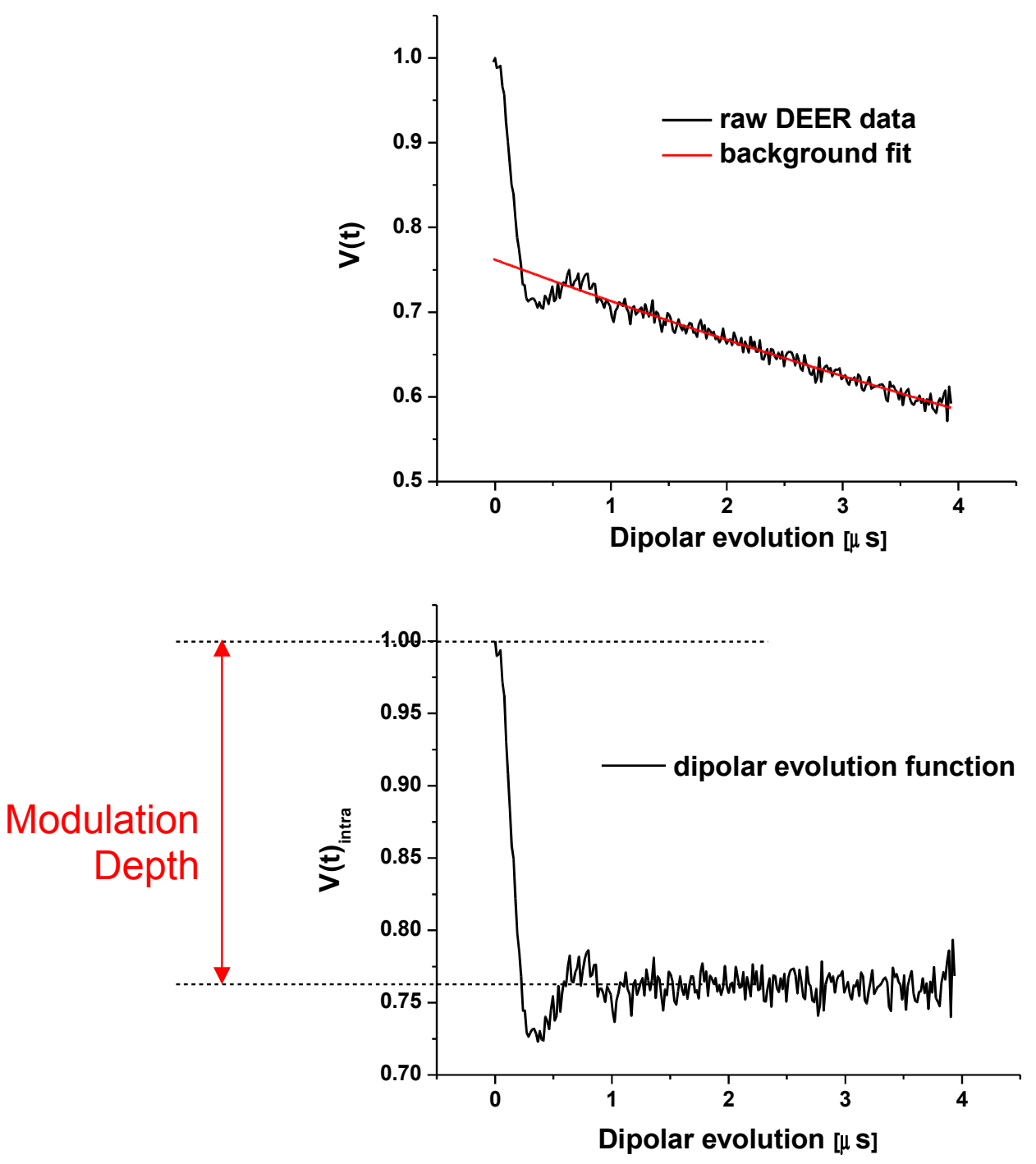

Figure 17. Processing of the DEER data. The raw data requires background subtraction (top) to extract intramolecular signal (bottom) that can be used to fit distance. The modulation depth is highlighted in red on the background-subtracted signal. 
approach however can be unreliable when analyzing higher amount of intermolecular distances or when the echo modulation has higher noise. An alternative approach is to use the Tikhonov regularization, that allows one to obtain the distance distribution directly from the time-domain dipolar evolution spectrum. For each distance distribution, a particular form factor $\mathrm{F}(\mathrm{t})$ is calculated:

$$
F(t)=K(t, r) P(r)
$$

where $\mathrm{P}(\mathrm{r})$ is the distance distribution and $\mathrm{K}(\mathrm{t}, \mathrm{r})$ is the kernel function, which is defined as:

$$
K(t, r)=\int_{0}^{1} \cos \left[\left(3 \mathrm{x}_{2}-1\right) \omega_{d d} t\right] d x
$$

with

$$
\omega(r)=\frac{2 \pi \cdot 52.04 \mathrm{MHz} n m^{-3}}{r^{3}}
$$

The echo modulation trace usually contains noise which, when fitted with with the regularization routine, would introduce artificial distance distribution. To counter this, the Tikhonov regularization features a smoothing factor $\alpha$, called a regularization parameter. The optimum distance distribution is found by fitting the following function:

$$
G_{\alpha}(P)=\|S(t)-D(t)\|^{2}+\alpha\left\|\frac{d^{2}}{d r^{2}} P(r)^{2}\right\|
$$

The optimum value of the regularization parameter needs to be chosen for each individual experimental result: lower values are applicable for low-noise traces with well defined distributions, whereas larger $\alpha$ values are needed when dealing with wider distance distributions. The figure $18 \mathrm{~A}$ shows that the parameter $\alpha$ directly affects the distributions and can be a source of result variability. 

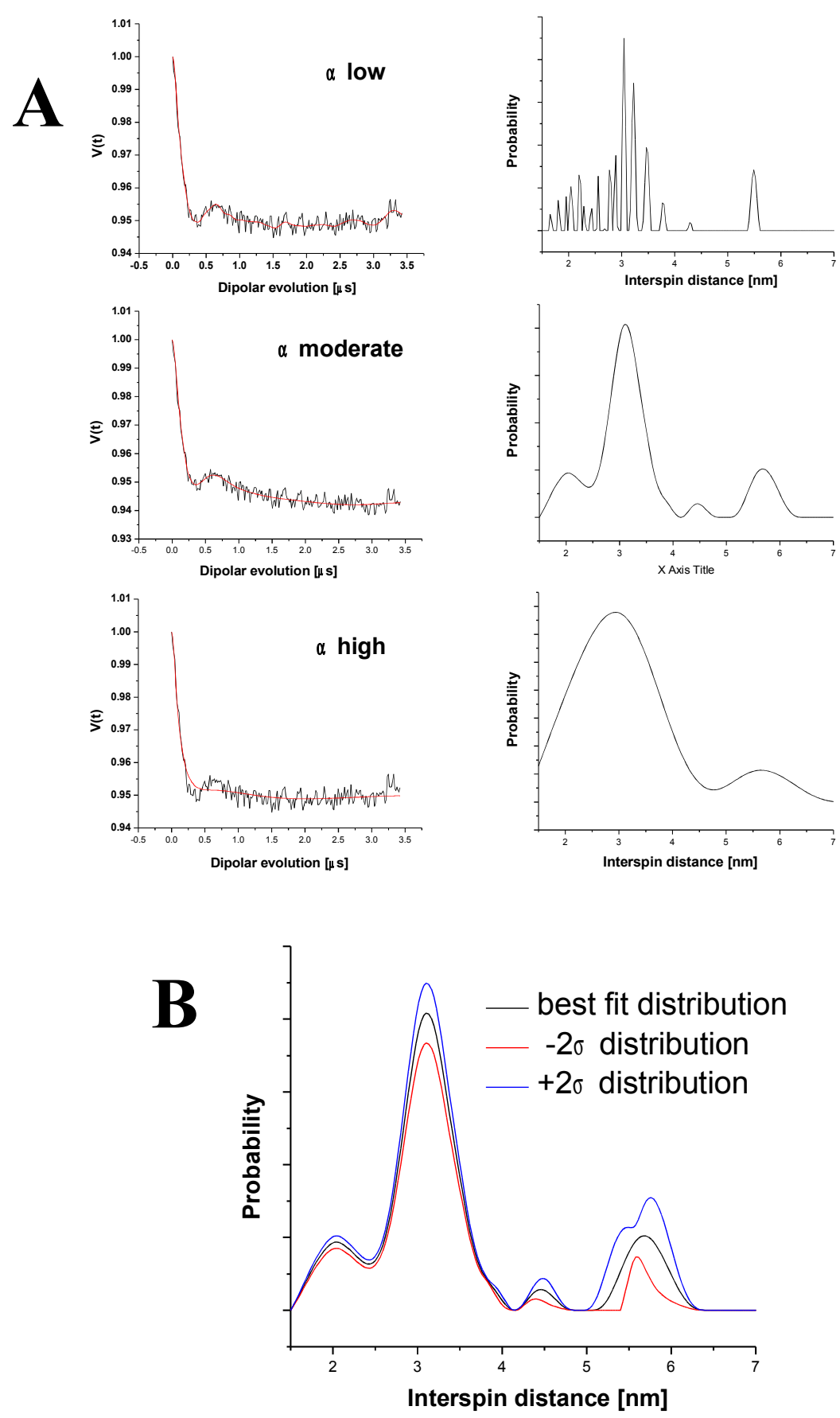

Figure 18. Computational processing of the DEER echo modulation.

A. The smoothing parameter of the Tikhonov regularization largely determines the number of discrete distances and their distributions. B. Successful baseline validation shows the average distance distribution, as well as the uncertainty of twice of the standard deviation of the distribution fit r.m.s.d. 
Another considerable source of distance distribution uncertainty is the imperfect background correction of the echo modulated spectrum. This becomes especially important for data containing information on longer distance, as a greater range of the pump pulse timing is required to fully characterize the oscillations of the echo modulation (equation 7). Oftentimes, a sufficiently long pump pulse time range is not possible to achieve, as the phase memory time for the spin-labeled system is not long enough to maintain the spin $x-y$ coherence. In this case the distance distributions from the fitting of a dipolar evolution spectrum will be compromised with uncertainty, though the mean distance will likely still be reliable, as long as the pump pulse timing range covers at least one echo oscillation. To analyze the amount of error due to imperfect background subtraction it is possible to perform a background validation routine, that involves fitting the background starting from different positions on the echo modulation. The start of the background is incremented in multiple steps, and multiple distance distributions are calculated. The distributions, which show the largest deviation from the experimental form factor are eliminated, and the ones that remain are analyzed statistically to provide the uncertainty to the final distance distribution. An example of distance distribution with an uncertainty obtained from a validation routine can be seen in Figure 18 B.

The distance distributions that are obtained will reflect the true conformational heterogeneity of the protein and the spin label sidechains. The work presented here features proteins labeled with the MTSL label; this label may add approximately 3-4 angstroms to the distance distribution. Other studies focused on engineering a label that is much more structurally constrained and does not 
artificially increase intermolecular distance distribution [92, 93].

\subsection{Power Saturation EPR}

The EPR experiment relies on the absorption of microwaves, which allows an unpaired spin to reach a higher energy level. The relative amount of low energy spins at thermal equilibrium is given by the Boltzmann distribution:

$$
\frac{n_{\alpha}}{n_{\beta}}=e^{-\Delta E / k T}=e^{-g \beta H / k T}
$$

where $n_{\alpha}$ and $n_{\beta}$ are the populations of the upper and lower energy states, respectively, $\mathrm{T}$ is the temperature and $\mathrm{k}$ is the Boltzmann constant. When the spins reach the higher energy state via microwave absorption, they will subsequently lose that energy to the environment, so that the spin ensemble will return to the thermal equilibrium. This process is called spin-lattice relaxation, and is specified by a rate constant $T_{1}$, that is defined as the rate at which the $\mathrm{z}$-axis component of the bulk magnetization vector (Figure $14 \mathrm{~B}$ ) decreases with time:

$$
\frac{d M_{z}}{d t}=\frac{-\left(M_{z}-M_{0}\right)}{T_{1}}
$$

There is a limited amount of microwave power that can be absorbed by the spins; if there is an excess of microwave power, then the spins will not be able to dissipate the energy through spin-lattice relaxation quickly enough, and the actual EPR signal will decrease (less spins will be able to absorb the microwaves, as more already are in the higher energy state). The power saturation technique, at its principle, measures the susceptibility of the spins to become saturated with microwave power. This in turn, can be easily modulated by introduction of paramagnetic species, which will interact with the spins, and increase the rate of the spin-lattice relaxation, delaying the onset of saturation (Figure 19 B). 
The system studied in this work is a protein that is anchored to the lipid bilayer, with segments of its cytoplasmic domain potentially contacting the lipid interface as well. It is therefore important to explain the effect of paramagnetic reagents on the spin-lattice relaxation of the nitroxide in the context of an aqueous-lipid interface. These reagents will be able to penetrate the bilayer as specified by the equation [95]:

$$
C_{i, m}(x)=C_{i, w} e^{\mu_{i, w}^{o} / R T} e^{-\mu_{i, m}^{o}(x) / R T}
$$

wher $C_{i, m}(x)$ is the concentration of a reagent $\mathrm{i}$ in the bilayer at a distance $\mathrm{x}$ from the interface, $C_{i, w}$ is the concentration of the reagent $i$ in the aqueous phase, and $\mu_{i, w}^{o}$ and $\mu_{i, m}^{o}$ are the standard state chemical potentials in the aqueous phase and in the bilayer, respectively. The above equation specifies a concentration gradient of a paramagnetic species that exists across the lipid bilayer (shown for oxygen and NiEDDA in Figure 19 A). This in turn indicates that the effect of the paramagnetic species on the nitroxide will vary depending on the latter relative position within the bilayer. As previously mentioned, the paramagnetic reagents will elevate the effective spin lattace relaxation rate, which occurs via the Heisenberg exchange mechanism. This mechanism involves an orbital overlap between the nitroxide and the paramagnetic species which allows the exchange of magnetization between the two, with the rate of:

$$
\mathrm{W}_{\mathrm{ex}}=\mathrm{k}_{\mathrm{ex}} \mathrm{C}_{\mathrm{r}}
$$

where $k_{\mathrm{ex}}$ is the exchange rate constant and $C_{r}$ is the concentration of the paramagnetic species (uniform for aqueous environment and specified by equation 25 for the lipid bilayer).

In the power saturation experiment, the peak-to-peak amplitude of the 
A

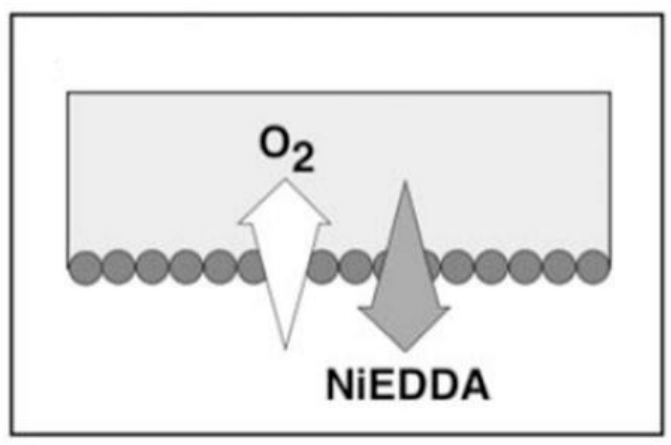

B

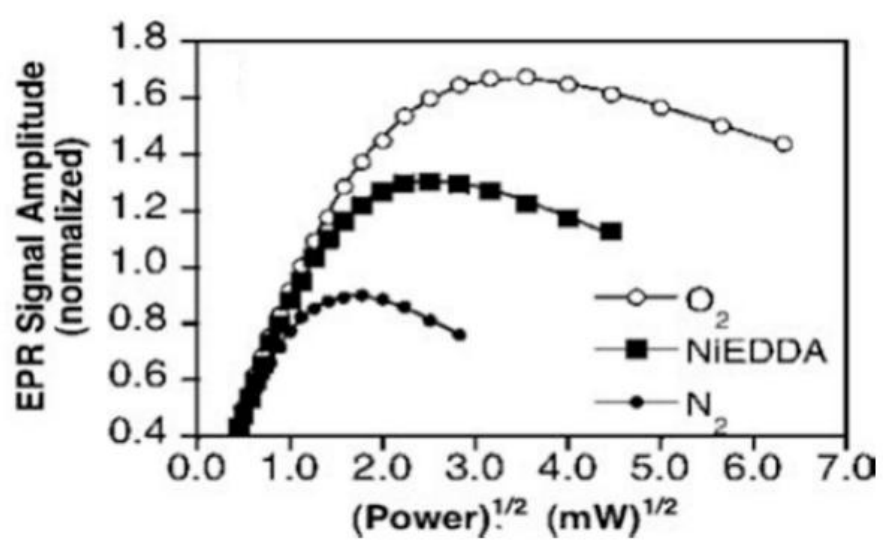

Figure 19. A. Opposite concentration gradients of NiEDDA and oxygen in aqueous lipid solution. B. Different saturation profiles due to presence of paramagnetic reagents [94]. 
central line is measured as a function of the microwave power.

In the absence of saturation, this amplitude has the form:

$$
\mathrm{A} \propto \mathrm{H}_{1} /\left(1+\mathrm{H}_{1}^{2} \gamma^{2} \mathrm{~T}_{1 \mathrm{e}} \mathrm{T}_{2 \mathrm{e}}\right)^{1.5}
$$

where $H_{1}$ is microwave magnetic field component, $\gamma$ is the gyromagnetic ratio, and $T_{1 e}$ and $T_{2 e}$ are spin-lattice and spin-spin relaxation times, respectively. The $H_{1}$ is proportional to the square root of the microwave power:

$$
\mathrm{H}_{1}=\Lambda \mathrm{P}^{1 / 2}
$$

where $\Lambda$ is a constant depending on the properties of the resonator. Saturation starts when the central line peak-to-peak amplitude no longer rises linearly with the square root of the microwave power. The parameter that specifies the saturation in respect to the microwave power is $\mathrm{P}_{1 / 2}$. It is defined as the microwave power at which the amplitude of the central line is half of the unsaturated value. When the paramagnetic reagent is added, the $\mathrm{P}_{1 / 2}$ changes in value according to equation:

$$
\Delta \mathrm{P}_{1 / 2}=\Delta \mathrm{P}_{1 / 2}-\Delta \mathrm{P}_{1 / 2}^{*}=\left(\left(2^{2 / 3}-1\right) /\left(\Lambda^{2} \gamma^{2} \mathrm{~T}_{2 \mathrm{e}}\right)\right) \mathrm{W}_{\mathrm{ex}}
$$

where $\Delta \mathrm{P}_{1 / 2}-\Delta \mathrm{P}_{1 / 2}^{*}$ are values obtained in the presence and absence of the paramagnetic and $\mathrm{T}_{2 \mathrm{e}}$ is the spin-spin relaxation time. To eliminate the effect of the resonator $(\Lambda$ value $)$ and of the spin-spin relaxation, the $\Delta \mathrm{P}_{1 / 2}$ is normalised against central line width (to account for the $\mathrm{T}_{2 \mathrm{e}}$ ) and against the quantity $\left[\Delta \mathrm{P}_{1 / 2} / \Delta \mathrm{H}_{\mathrm{pp}}\right]$ of the 2,2-diphenyl-1-picrylhydrazyl (DPPH). The DPPH is a useful radical standard owing to its high stability and its well characterized absorption profile [96]. The normalised accessibility parameter, termed $\Pi$, is calculated in an equation:

$$
\Pi=\left(\Delta \mathrm{P}_{1 / 2 /} \Delta \mathrm{H}_{\mathrm{pp}}\right) /\left(\Delta \mathrm{P}_{1 / 2} / \Delta \mathrm{H}_{\mathrm{pp}}\right)_{\mathrm{DPPH}}
$$

The final parameter that combines the accessibility parameters from two paramagnetic reagents is termed the depth parameter $\Phi$ : 


$$
\Phi=\ln \left(\frac{\Pi_{I}}{\Pi_{I I}}\right)
$$

This parameter directly relates the chemical potentials of both paramagnetic species at any depth of the bilayer. By recording several depth parameters for lipids with spin labels at different positions it is possible to obtain a standard curve that shows a fairly linear dependance of $\Phi$ on the nitroxide position in the bilayer (Figure 20).

Aside from the depth parameter $\Phi$, the accessibility parameter $\Pi$ can on its own provide useful information on the protein system. The parameter $\Pi$ recorded for a particular paramagnetic species will vary depending on the relative accessibility of the nitroxide, irrespective of the proximity to the lipid bilayer. When the spin label is introduced into a relatively exposed site (like a surface of a helix) the collision rate $\mathrm{W}_{\mathrm{ex}}$ will be higher compared to the spin label buried inside the protein. This is due to the parameter $\mathrm{k}_{\mathrm{ex}}$ from the equation 27 being a function of any possible steric hindrance. In addition, binding of another protein to the labeled site will also yield measurable decrease in the collision rate with a paramagnetic. The $\Pi$ parameter can therefore be used to characterize the accessibility of a spin label to the solvent, which can provide insight on protein-protein interactions . 


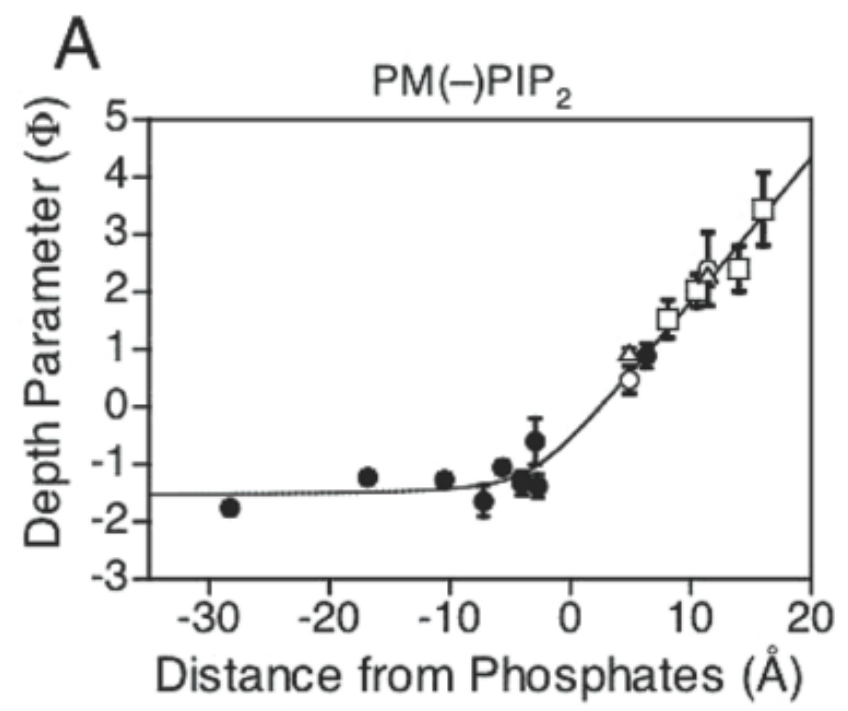

Figure 20. Depth parameter dependance on the lateral position across the lipid membrane.

An example of a calibration curve, obtained from several experiments with spin labeled lipids. Such curve can then be used to estimate the position of the proteinintegrated nitroxide relative to the interface. Note that the calibration curve is only useful for a specific lipid composition, as the chemical gradient of paramagnetic species will vary with different bilayers. Figure taken from reference 97. 
Chapter 3: Allosteric Control of Syntaxin 1a by Munc18-1: Characterization of the Open and Closed Conformations of Syntaxin 


\subsection{ABSTRACT}

Syntaxin $1 \mathrm{a}$ is a plasma membrane soluble $\mathrm{N}$-ethylmaleimide-sensitive factor attachment receptor protein (SNARE) that contains an H3 domain (SNARE motif) and a regulatory Habc domain. These regions associate to produce a closed state, which is generally thought to suppress assembly of syntaxin into the SNARE complex. However, the molecular nature of the closed and open states of syntaxin is not well defined. Here, we use electron paramagnetic resonance spectroscopy to characterize conformational exchange in syntaxin. The data indicate that the $\mathrm{H} 3$ segment is in equilibrium between ordered and disordered states that have significant populations. In solution, the central region of the H3 segment is positioned close to the Habc domain and the configuration of syntaxin 1a is dominated by a closed state. However, an open state is enhanced in full-length membrane reconstituted syntaxin. Munc18-1 binding alters the equilibrium along H3 to favor the ordered, folded state. Munc18 also suppresses the minor open population and narrows the distance distributions between H3 and Habc. The allosteric control exhibited by Munc18 on the H3 segment and the suppression of the minor open component may both play a role in regulating membrane fusion by controlling the assembly of syntaxin into the SNARE complex.

\subsection{INTRODUCTION}

Neurotransmitter release is mediated by a membrane fusion event that joins the synaptic vesicle membrane with the presynaptic plasma membrane. This process is mediated by soluble $\mathrm{N}$-ethylmaleimide-sensitive factor attachment receptor proteins (SNAREs), which assemble into a tight four helical bundle that is thought to 
provide the energy required to overcome the barrier to fusion $[31,101]$. In the neuronal system, the helical SNARE complex is formed from three proteins: syntaxin 1a and SNAP-25 in the plasma membrane and synaptobrevin in the vesicle membrane. The regulation and assembly of these proteins to form the SNARE bundle is essential to neuronal fusion, and a number of critical effector proteins function to mediate this process. Sec1/Munc18 (SM) proteins are conserved regulators of the membrane fusion process $[44,102,103]$, and in the neuronal system they include Munc18-1, which interacts with syntaxin 1a and is believed to play a role in regulating syntaxin availability and assembly into the SNARE complex.

Munc18-1 is thought to have two modes of interaction with syntaxin 1a. One mode of binding is based upon a crystal structure for the Munc18-1-syntaxin complex $[43,63]$, shown in Fig. $21 \mathrm{c}$. In this structure, the concave surface formed by domains 1 and 3a of Munc18-1 interacts with both the SNARE forming (H3) and regulatory (Habc) domains of syntaxin 1a. This structure is termed closed because the $\mathrm{H} 3$ segment is in contact with the Habc domain and syntaxin 1a is inhibited from assembling into the SNARE complex [63]. Munc18-1 has a second mode of binding in which it interacts with syntaxin 1a in an open conformation. In this mode, the H3 segment of syntaxin 1a is assembled into a binary or ternary SNARE complex, and the H3 domain must be dissociated from the Habc domain $[46,65]$. In this open state, the interaction of Munc18-1 is mediated by an Nterminal peptide in syntaxinla that precedes the Habc domain. This interaction is thought to have a stimulatory role in fusion, although recent work indicated that it may not be critical for synaptic transmission [104]. 


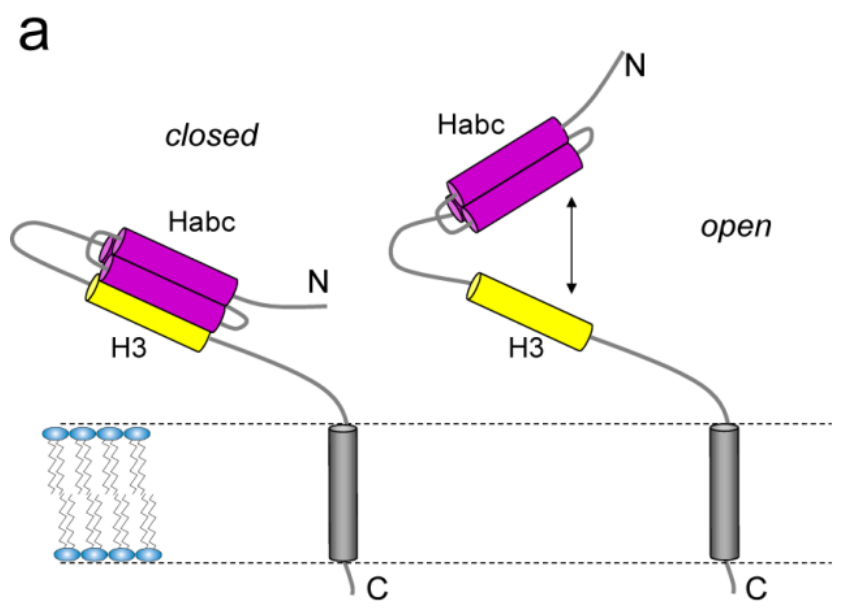

b

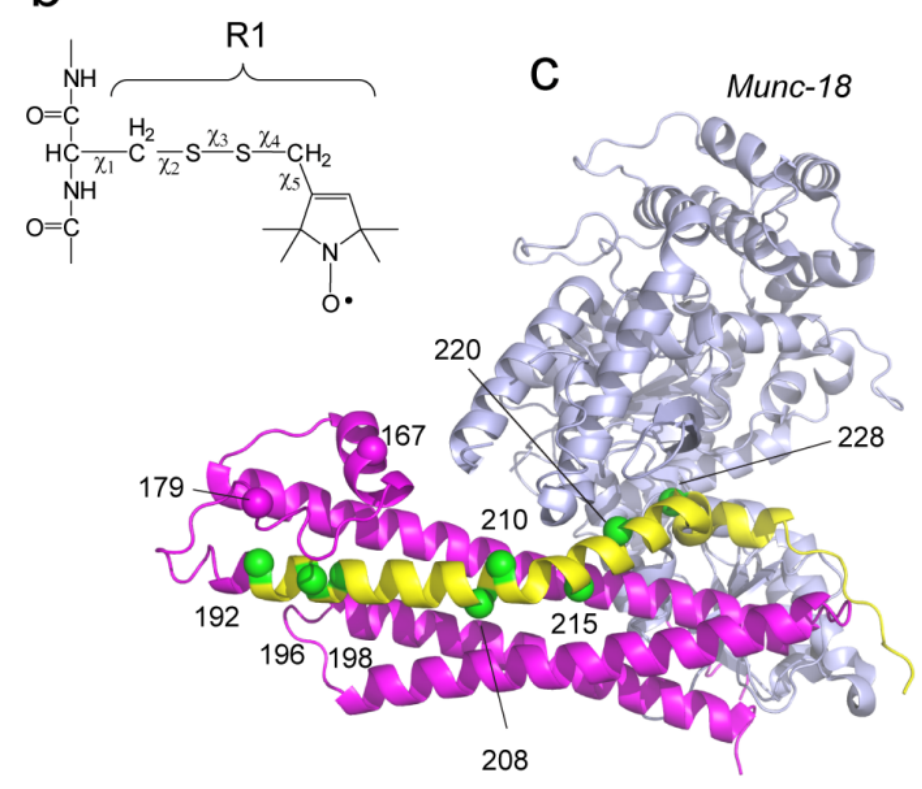

Figure 21 (a) Syntaxin 1a is a membrane-anchored protein containing a SNARE motif (H3 segment in yellow) and regulatory Habc domain (magenta) that undergoes a closed-to-open transition regulated by Munc18-1. (b) The spin-labeled side-chain R1 shows the rotatable bonds linking the nitroxide to the protein backbone. (c) The R1 side chain was attached to several sites along H3, which are shown in the crystal structure of the syntaxin 1a/Munc18-1 complex (PDB ID: 3C98). The $\mathrm{C} \alpha$ carbons to which $\mathrm{R} 1$ has been attached are rendered as green spheres. 
At the present time, information on the dynamics and structures assumed by syntaxin 1a either alone or in complex with Munc18-1 is limited. It is generally thought that the $\mathrm{H} 3$ domain is flexible but assumes a more ordered helical structure upon Munc18-1 binding [4, 40]. In the absence of Munc18-1, syntaxin 1a may fluctuate between open and closed forms, as shown in Fig. 21 a, but reports on this conformational equilibrium differ. A single-molecule fluorescence study indicated that syntaxin adopts a predominantly open conformation, with a minor population in the closed state [40]. Addition of Munc18-1 shifts this equilibrium shifts to the closed state, with the interdomain distances closely matching the crystal structure (Fig. $21 \mathrm{c}$ ). In contrast, an NMR study of syntaxin 1a indicated that the protein is predominantly closed, and the H3 domain is closely associated with the Habc domain whether Munc18-1 is present or not [4]. A more recent study using x-ray and neutron diffraction differed from these two studies and concluded that the syntaxin 1a/Munc18-1 complex is not represented by the crystal structure, and that Munc18-1 does not close syntaxin 1a unless the N-terminal segment of syntaxin is removed [105].

Protein dynamics and structural fluctuations play important roles in regulating protein-protein interactions $[106,107]$. As a result, dynamics and conformational exchange in syntaxin are likely to be important properties that define the ability of syntaxin to assemble into the SNARE complex and drive membrane fusion. However, dynamics and conformational exchange on the timescale that is important for protein recognition can be difficult to characterize.

Electron paramagnetic resonance (EPR) spectroscopy is particularly well suited to examine conformational exchange events in proteins, which typically 
occur on the microsecond timescale [108-110]. In this work, we use site-directed spin labeling (SDSL) along with continuous-wave and pulse EPR spectroscopy to characterize conformational exchange in syntaxin 1a in the presence and absence of Munc18-1. The H3 segment of syntaxin 1a is found to be in conformational exchange between ordered and disordered states, both of which have significant populations. In the absence of Munc18, the central region of the H3 segment remains positioned near the Habc domain, and only a minor population in this central segment samples an open configuration. This open state is enhanced in fulllength membrane reconstituted syntaxin. The binding of Munc18 shifts the conformational equilibria along $\mathrm{H} 3$ toward a more ordered state and eliminates the open configuration. These changes represent an allosteric control of the H3 domain structure and suggest that dynamic disorder at the N-terminal end of the $\mathrm{H} 3$ segment may modulate the assembly of syntaxin into binary or tertiary SNARE complexes.

\subsection{MATERIALS AND METHODS}

\section{Mutagenesis, expression, and purification}

pET28 plasmids bearing the soluble syntaxin-1a (residues 1-262), full-length syntaxin-1A (residues 1-288), and Munc18-1 genetic inserts were provided by Reinhard Jahn and Dirk Fasshauer (Max Planck Institute for Biophysical Chemistry, Gottingen, Germany). The QuikChange PCR method (Agilent Technologies, Wilmington, DE) was used to engineer single and double cysteine mutations into syntaxin 1a, and a BL21 Codon-Plus DE3 (RIL) Escherichia coli host (Agilent Technologies) was used for expression. The cells were grown in 
lysogeny broth (LB) media with $40 \mathrm{mg} / \mathrm{L}$ kanamycin. When the cells reached an optical density (o.d.) of $0.8-1.0$, expression was induced with $0.4 \mathrm{mM}$ of isopropylthio- $\beta$-galactoside. The cells were incubated at $20^{\circ} \mathrm{C}$ overnight and then harvested by centrifugation at $3500 \mathrm{~g}$.

For protein purification, the cell pellet was resuspended in the buffer containing $20 \mathrm{mM}$ HEPES, $500 \mathrm{mM} \mathrm{NaCl}, 8 \mathrm{mM}$ imidazole, $20 \mu \mathrm{mol}$ leupeptin, 50 $\mu$ mol 4-(2-Aminoethyl) benzenesulfonyl fluoride hydrochloride, $750 \mathrm{U}$ benzonase nuclease (EMD Millipore, Billerica, MA), pH 7.3. For full-length syntaxin, sodium cholate $5 \%(\mathrm{w} / \mathrm{v})$ was added to this buffer, and $1 \%(\mathrm{w} / \mathrm{v})$ sodium cholate was present in subsequent buffers used during purification. The cells were passed through a French press twice and centrifuged at 90,000 g for $1 \mathrm{~h}$. The supernatant was then mixed with $5 \mathrm{ml}$ of preequilibrated NiNTA resin (Biorad, Hercules, CA), shaken for $1 \mathrm{~h}$ at $4^{\circ} \mathrm{C}$, and subsequently washed with 10 volumes of buffer containing $20 \mathrm{mM}$ HEPES, $500 \mathrm{mM} \mathrm{NaCl}, 20 \mathrm{mM}$ imidazole, $\mathrm{pH}$ 7.3. For both soluble and full-length syntaxin, the first four volumes contained $1 \%(\mathrm{w} / \mathrm{v})$ Triton X-100 and the next four contained $1 \%(\mathrm{w} / \mathrm{v})$ sodium cholate. The protein was

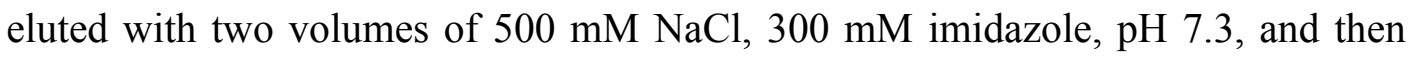
dialyzed against $20 \mathrm{mM}$ HEPES, $500 \mathrm{mM} \mathrm{NaCl}, 2$ mM EDTA, 2 mM dithiothreitol, $\mathrm{pH}$ 7.3, followed by dialysis against a buffer with $20 \mathrm{mM}$ HEPES, $150 \mathrm{mM} \mathrm{NaCl}$, $\mathrm{pH}$ 7.3. During dialysis, $250 \mathrm{U}$ of thrombin were added to the protein to cleave the N-terminal His-tag. Protein purity was assessed by SDS-PAGE and the protein solution was concentrated by ultrafiltration (Ultracel 30K; EMD Millipore).

For syntaxin spin labeling, $1 \mathrm{mg}$ of 1-oxyl-2,2,5,5-tetramethyl-3-pyrroline3-methyl methanethiosulfonate (MTSL; Toronto Research Chemicals, Toronto, 
Canada) was added in ethanol and the protein was incubated overnight at $4^{\circ} \mathrm{C}$. The unbound spin label was removed using a HiPrep 26/10 desalting column (GE HealthCare, Piscataway, NJ), and the eluted protein was concentrated by ultrafiltration.

\section{Sample preparation and EPR measurements}

To prepare soluble syntaxin (1-262) with or without Munc18-1 for EPR, protein samples were mixed with $0.5 \mathrm{ml}$ of desired buffer and concentrated by ultrafiltration (Ultracel 30K; EMD Millipore). These buffers were typically $20 \mathrm{mM}$ phosphate or MOPS buffers at low to moderate ionic strengths (see figure legends). Samples for continuous-wave EPR ranged from 10 to $100 \mu \mathrm{M}$, and samples for pulse EPR were typically used at a concentration of $40 \mu \mathrm{M}$ but were recorded in a few cases at concentrations as low as $2 \mu \mathrm{M}$.

To prepare full-length syntaxin (1-288), a lipid film of either POPC or POPC/POPS (3:1) (Avanti Polar Lipids, Alabaster, AL) was rehydrated in a $20 \mathrm{mM}$ phosphate buffer ( $\mathrm{pH}$ 7.3) with $12 \mathrm{mM}$ of sodium cholate to a total lipid concentration of $15 \mathrm{mM}$. Syntaxin (1-288) was added to this mixture and the solution was then incubated for half an hour at room temperature and dialyzed twice against buffer. The resulting vesicles were centrifuged at 500,000 g for 20 min and the vesicle pellet was dissolved in buffer. The protein/lipid ratio was 1:1000 and the protein concentration in the sample was $50-100 \mu \mathrm{M}$.

For continuous-wave measurements, samples were loaded into glass capillaries with $0.6 \mathrm{~mm}$ inner diameter (i.d.) and $0.84 \mathrm{~mm}$ o.d. (VitroCom, Mountain Lakes, NJ). EPR spectra were recorded using a Varian E-line 102 Century series X-band spectrometer fitted with a loop-gap resonator (Medical 
Advances, Milwaukee, WI) or a Bruker EMX spectrometer with a roomtemperature ER 4123D dielectric resonator. The measurements were carried out at 2 $\mathrm{mW}$ incident microwave power using $1 \mathrm{G}$ modulation amplitude. The spectra were then processed using LabVIEW programs provided by Christian Altenbach (University of California, Los Angeles, CA).

For double electron-electron resonance (DEER) measurements, protein samples were loaded into quartz capillaries $(2.0 \mathrm{~mm}$ i.d. and $2.4 \mathrm{~mm}$ o.d.) and frozen in a dry-ice-isopropanol bath. The pulse experiments were carried out at 80 K on a Bruker Elexsys E580 spectrometer at X-band using an ER4118X-MS3 splitring resonator or at Q-band using an EN5107D2 dielectric resonator (Bruker Biospin, Billerica, MA). Data in pulse mode were acquired using a four-pulse DEER sequence [111] with $16-n s \pi / 2$ and $32-n s ~ \pi$ observe pulses separated by a $32-$ ns $\pi$ pump pulse. The dipolar evolution times were typically $2-4 \mu$ s. The pump frequency was set to the center maximum of the nitroxide spectrum and the observe frequency was set to the low-field maximum, typically $15-25 \mathrm{MHz}$ higher in frequency. The dipolar evolution data were processed and distance distributions determined using Tikhonov regularization incorporated into the DeerAnalysis2011 software package [112]. This program contains an error analysis routine that was used to assess the error produced by background subtraction upon the distance distribution

\section{Syntaxin 1a EPR spectra in sucrose}

Syntaxin EPR spectra were titrated with $0-40 \%$ (w/v) sucrose (Fisher Scientific, Pittsburgh, PA) while the final protein concentrations were maintained at $\sim 40 \mu \mathrm{M}$. 
In a few cases, syntaxin (1-262) was maintained on the NiNTA through its His ${ }_{6}$ tag and titrated with sucrose. The spectra displayed two motional components, which varied in population as a function of sucrose concentration. The percentage of each motional population in these spectra was determined by fitting the spectra with the MOMD model [79] as implemented in MultiComponent, a LabVIEW program provided by Dr. Christian Altenbach (University of California, Los Angeles, CA). Additional details regarding the fitting are provided in Fig. S2 of the Supporting Material). Generation of syntaxin 1a models from distance restraints and simulated annealing Models for the open configuration of syntaxin 1a were generated using Xplor-NIH $[113,114]$ in a manner similar to that described previously [21]. Briefly, the spin-label side-chain R1 was appended to the crystal structure of syntaxin 1a at appropriate locations and a restraint was applied to each spin label so that the $\mathrm{C} \alpha$ proton-S $\delta$ distance was $2.6 \pm 0.1 \AA$. This restraint places the label in conformations that have been observed experimentally $[115,116]$. The long, open distances between $\mathrm{H} 3$ and Habc obtained from analysis of the DEER data were chosen as restraints and included a range that represented $2 / 3$ of a standard deviation (SD) in the distribution. Simulated annealing was performed using these restrains while allowing all side chains to be free, including $\mathrm{R} 1$, and allowing the region that connects the $\mathrm{H} 3$ and Habc segments (residues 157-189) to be flexible. The backbone atoms of the $\mathrm{H} 3$ and Habc regions were fixed. Structures were visualized and analyzed with the program PyMOL (DeLano Scientific LLC, Palo Alto, CA).

\subsection{RESULTS}

Oligomerization of syntaxin (1-262) 
Syntaxin 1a has a tendency to oligomerize through its $\mathrm{H} 3$ domain $[38,117]$, and the syntaxin (1-262) fragment used here was examined to determine under what conditions it oligomerizes or is monomeric in solution. Two different approaches, dynamic light scattering (DLS) and NMR, were used to examine the effective hydrodynamic radius of syntaxin (1-262). Under conditions of moderate or low ionic strength, both DLS and the one-dimensional TRACT experiment [118] yielded an effective hydrodynamic radius of $\sim 2.5-3 \mathrm{~nm}$. These data (see Fig. S1) are consistent with the expected behavior of the syntaxin monomer, but do not rule out the presence of some oligomer. Pulse EPR is sensitive to the formation of specific oligomers (dimer or trimer) and the formation of larger aggregates [119]. Relaxation-time measurements indicated that larger aggregates did not occur in these samples, and under conditions of low salt, no significant coherent dipolar interactions were observed that would indicate oligomerization. However, as observed previously [4], evidence of aggregation was observed at higher protein concentrations and ionic strengths, and appeared as a significant modulation in the DEER signal (Fig. S1). At or below physiological ionic strength and at moderate to low protein concentrations $(10-50 \mu \mathrm{M})$, the majority of the protein is monomeric, but some level of dimer appears to be present $(\sim 10-25 \%)$. Additional data characterizing the sample and quantitating the oligomeric state of syntaxin (1-262) are included in the Supporting Material.

The H3 domain is in conformational exchange between ordered and disordered forms.

Shown in Fig. 22 are 10 EPR spectra obtained from single labels at several points along the $\mathrm{H} 3$ domain of syntaxin $1 \mathrm{a}$ (Fig. $1 \mathrm{c}$ ), as well as a region linking $\mathrm{H} 3$ to the 


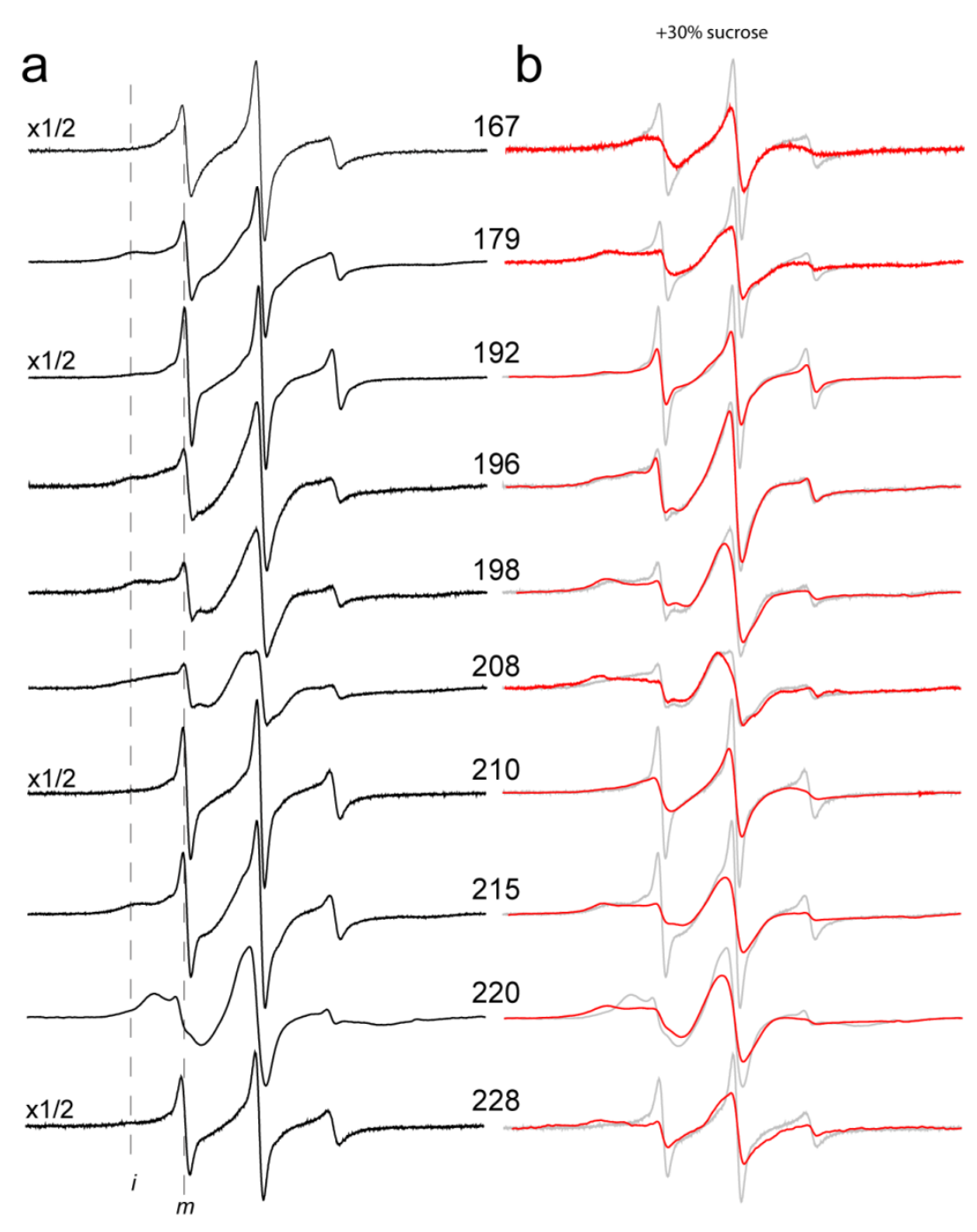

Figure 22 ( $a$ and b) X-band EPR spectra for selected sites in the Habc/H3 linker and along the $\mathrm{H} 3$ segment of syntaxin (1-262) in solution (a) and in the presence or absence (gray traces) of $30 \%$ sucrose (b). Many of the spectra result from R1 labels having at least two motional components, and the position of hyperfine resonances that result from mobile (m) and immobile (i) R1 side chains is indicated. The presence of sucrose alters the populations of these components, indicating that a protein conformational equilibrium is the source of these components. Spectra were recorded at concentrations ranging from 10 to $100 \mathrm{mM}$ in $139 \mathrm{mM} \mathrm{KCl}, 12 \mathrm{mM} \mathrm{NaCl}, 20 \mathrm{mM}$ MOPS, $\mathrm{pH}=7.3$. The spectra are 100 Gauss scans and have been normalized to total spin number. 
Habc domain. In almost all cases, the EPR spectra reflect at least two modes of motion of the spin-labeled side-chain R1 (Fig. 21 b). One motional component is characteristic of an unfolded protein segment (labeled $\mathrm{m}$ in Fig. 22 a), where the label executes isotropic label motion on the order of 1 ns or less. A second component (indicated by i) results from an $\mathrm{R} 1$ side chain that is either in tertiary contact or attached to a folded protein segment. For example, the spectrum from site 210 is a composite of two motional components, where one component $(\sim 35 \%$ of the signal) originates from R1 having isotropic motion with a correlation time, $\tau_{\mathrm{c}}$, of $\sim 0.6 \mathrm{~ns}$, and the second one ( $\sim 65 \%$ of the signal) having a $\tau_{\mathrm{c}}$ of $2.7 \mathrm{~ns}$. The slower-moving component is characteristic of that obtained from a dynamic helical region. Similarly, $\sim 30 \%$ of the signals at sites 192 and 228 are characteristic of unfolded protein, and at site 215 , the unfolded component represents $10 \%$ of the total signal. The EPR spectra at sites 196, 198, and 208 indicate that these labels are in tertiary contact with $5 \%$ or less of the signal originating from an unstructured or dynamic protein backbone. These three labels are at sites that are expected to be in tertiary contact based upon the closed state as defined by the sytaxin1a/Munc18-1 crystal structure (PDB ID: 3C98) shown in Fig. 21 c. Additional details regarding simulations of these EPR spectra are provided in Fig. S2.

The multiple modes of R1 motion seen in Fig. 22a may arise either from different rotameric states of the R1 side chain or from two different protein conformations. One approach to establish the source of these motional components is to examine the EPR spectra in the presence of a stabilizing osmolyte, such as sucrose. Osmolytes will alter the energetics between conformers that have different solvent exposures [108], but they will not alter R1 rotameric equilibria [109]. As 
can be seen in Fig. 22 b, sucrose addition alters the EPR spectra at most positions to populate the less mobile component, and these changes are reversible upon removal of sucrose. This result indicates that the motional components seen in these EPR spectra represent different protein conformations, and that these conformations are in equilibrium.

EPR spectra for five positions along the H3 domain were titrated as a function of sucrose, and the equilibrium constant was determined at each point assuming a two-state model in which the label is responding to both folded and unfolded protein conformations. From the equilibrium constant, the conformational free energy was plotted as a function of sucrose concentration and yielded a linear dependence as would expected for a two-state conformational transition (see Fig. S3). The slopes of these plots are similar, indicating that the hydrated surface area change that occurs along this length of the H3 domain is similar [120]. Several titrations with sucrose were carried out for syntaxin (1-262) attached to beads. These yielded similar spectral changes, indicating that the structural changes produced by sucrose for the syntaxin (1-262) construct were not the result of protein oligomerization (Fig. S3)

These EPR spectra reveal several features of the H3 segment. First, the effects of sucrose demonstrate that the H3 segment and the linker connecting H3 with Habc are in conformational exchange. Second, labels at exposed helical surfaces in the crystal structure (sites $167,192,210$, and 228) indicate that $\mathrm{H} 3$ is in equilibrium between folded and unfolded forms but is dominated by a folded form. Finally, sites in which the EPR spectra reflect tertiary contact of the R1 side chain (e.g., sites 198, 208, and 215) are in contact with the Habc segment in the crystal 
structure, suggesting that these regions of the H3 segment are closely associated with the Habc domain. This indicates that syntaxin 1a does not spend a significant fraction of time in an open configuration where the $\mathrm{H} 3$ segment is significantly displaced from the Habc segment in the absence of Munc18.

\section{Munc18 induces an ordering in the $\mathrm{H3}$ segment and allosterically modulates the configuration of $\mathrm{H3}$.}

EPR spectra from several sites within and outside the H3 segment are shown in Fig. $23 \mathrm{a}$ in the absence and presence of Munc18. Shown in Fig. $23 \mathrm{~b}$ are the scaled mobilities (Ms) for these spectra, which provide a relative measure of R1 side-chain motion (see legend to Fig. 23). The addition of Munc18 reduces the motional averaging of labels along H3. The R1 side chain at sites 220 and 228 should interact with Munc18, and the reduction in motion, particularly at site 228, is consistent with this expectation. Spectra from sites C-terminal to the Munc18-binding region (sites 247, 254, and 259) are not strongly modulated by Munc18 binding and the line shapes are characteristic of a disordered protein segment. At sites that should contact the Habc domain in the closed state (sites 198, 208, and 215), Munc18 binding results in the appearance of wide, well-resolved hyperfine features consistent with the formation of a compact closed state of syntaxin. Site 210 is an exposed helical site in the crystal structure, and the bound EPR spectrum is consistent with spectra that are obtained from helical sites, indicating that Munc18 increases helical content at this site. At site 192, which is remote from the Munc18binding site, the EPR spectra show that Munc18 induces folding at this site and increases the fraction of 192R1 that is in contact with the H3/Habc linker region. 




Figure 23 (a) X-band EPR spectra for sites along the H3 segment in the absence (gray traces) and presence of Munc18-1. The arrows indicate the position of wellresolved components of the hyperfine interaction, which result from immobilization of the labeled side chain on a timescale of tens of nanoseconds. R1-labeled syntaxin (1-262) was used at concentrations of $10-40 \mu \mathrm{M}$ in $139 \mathrm{mM} \mathrm{KCl}, 12 \mathrm{mM} \mathrm{NaCl}, 20$ $\mathrm{mM}$ MOPS, $\mathrm{pH}$ 7.3. The spectra are 100 Gauss scans and have been normalized to total spin number. (b) Scaled mobilities (Ms), determined from the spectra in panel 
a as described previously [99], range from 0 to 1 , where 0 represents the least mobile and 1 represents the most mobile spectra seen in proteins (gray bars in the absence of Munc18-1). The values of Ms are primarily dependent upon the correlation time of the label [124], and in multicomponent spectra, such as those shown here, the mobile lineshape will tend to dominate Ms. 
This result suggests that the $\mathrm{N}$-terminal region of the $\mathrm{H} 3$ domain of syntaxin is allosterically modulated by Munc18, a process that may be important for regulating the assembly of syntaxin into the SNARE complex.

The central portion of $\mathrm{H3}$ is closely associated with the Habc domain even in the absence of Munc18.

The EPR spectra in Fig. 22 indicate that the H3 domain is in contact with the Habc domain in the absence of Munc18. To confirm that this is the case, and to determine what fraction of the $\mathrm{H} 3$ segment might be dissociated from the Habc domain (in an open configuration), we used DEER to measure dipolar interactions between pairs of labels, with one label being placed within the H3 segment and the other within the Habc domain. The spin pairs, which are shown in Fig. 24 a, were chosen so that the label would have minimal interference with the closed state of syntaxin. Using the four-pulse DEER experiment [111], we determined the distances and distance distributions for five spin pairs. Shown in Fig. 24, b and c, are data for two of these spin pairs, 52R1/210R 1 and $105 \mathrm{R} 1 / 226 \mathrm{R} 1$, and a summary of the data is presented in Table 1.

The spin pair 52R1/210R1 places two labels on exposed helical surface sites so that the interspin vector bridges between the central portions of the $\mathrm{H} 3$ and Habc domains. As shown in Fig. 24 b, the DEER signal yields one major distance with a broad distribution centered at $\sim 32 \AA$ and a minor distance at $49 \AA$ that represents $11 \%$ of the signal. The broad distribution at $32 \AA$ suggests that the $\mathrm{H} 3$ segment is conformationally heterogeneous, a result that is consistent with the continuouswave spectra from 210R1 (Fig. 22). If the spin labels are placed into the crystal 
a

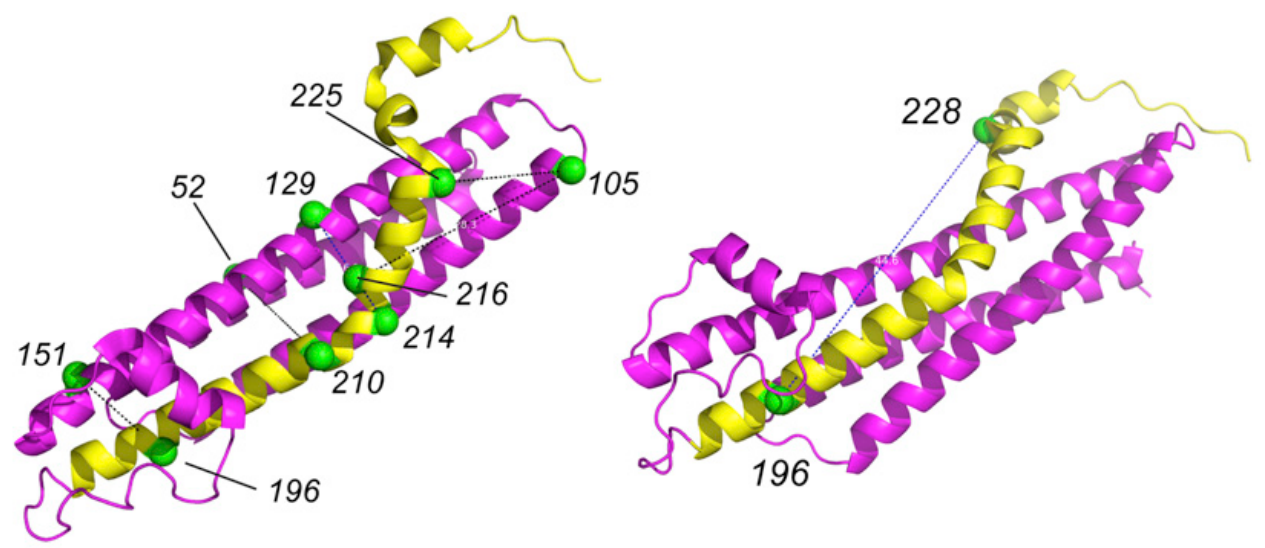

b

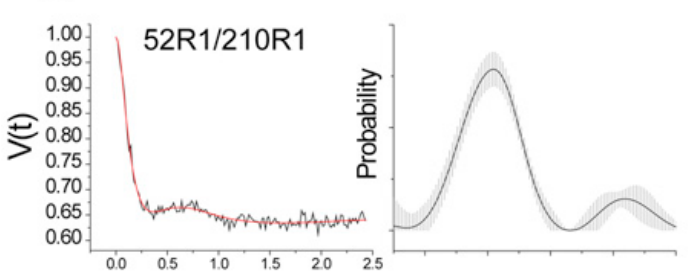

C
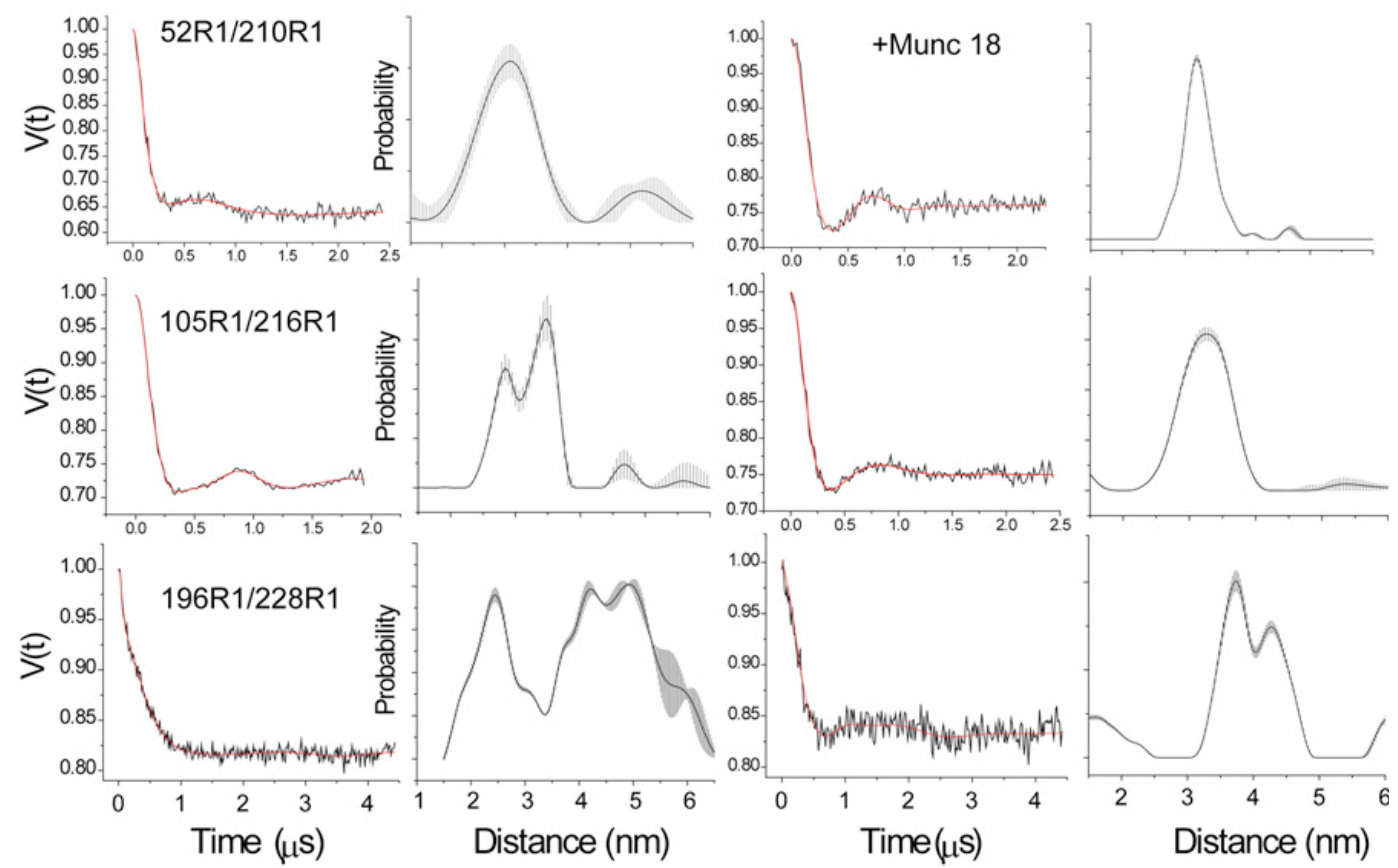

Figure 24 DEER data for three spin pairs in syntaxin 1a (1-262). (a) Crystal structure of syntaxin 1a from the Munc18-1/syntaxin 1a crystal structure (PDB ID: 3C98), showing the positions of six spin pairs examined here. One spin pair (196R1/228R1) is positioned along the H3 segment. (b) DEER signals and distance 
distributions for syntaxin 1a in solution, where the red traces represent the best fits to the dipolar evolution that was used to generate the distribution. (c) DEER signals for the same spin pairs shown in panel $b$ in the presence of Munc18-1. The shaded regions in the probability distributions represent the range of solutions that can be achieved by variation of background subtraction (due to intermolecular spin interactions). These include all fits having root mean-square deviation values within $15 \%$ of the best fit. These double-R1-labeled syntaxin (1-262) mutants were used at a concentration of $40 \mu \mathrm{M}$ in $139 \mathrm{mM} \mathrm{KCl}, 12 \mathrm{mM} \mathrm{NaCl}, 20 \mathrm{mM}$ MOPS, $\mathrm{pH}=7.3$. 


\begin{tabular}{|c|c|c|c|c|}
\hline & \multicolumn{2}{|r|}{ Syntaxin } & \multicolumn{2}{|c|}{ Syntaxin + Munc18 } \\
\hline & Major & Minor & Major & Minor \\
\hline Syx 151R1/196R1 & $23, \sigma 3.1$ & $34, \sigma 2.5(14 \%) 45, \sigma 2.0(12 \%)$ & $25, \sigma 3.0$ & $37, \sigma 2.0(13 \%) 45, \sigma 1.9(10 \%)$ \\
\hline Syx 52R 1/R210R 1 & $30, \sigma 8.8$ & $49, \sigma 5.1(11 \%)$ & $32, \sigma 4.6$ & $46, \sigma 3(2 \%)$ \\
\hline Syx 129R1/214R1 & $25, \sigma 8$ & $45, \sigma 1.8(10 \%)$ & $25, \sigma 6$ & $44, \sigma 5(5 \%)$ \\
\hline Syx 105R1/216R1 & $35, \sigma 3.6(65 \%)$ & $47, \sigma 3(6 \%)$ & $32, \sigma 7$ & - \\
\hline Syx $105 \mathrm{R} 1 / 225 \mathrm{R} 1$ & $\begin{array}{l}20, \sigma 7(44 \%) \\
28, \sigma 5(22 \%)\end{array}$ & $45, \sigma 3(10 \%)$ & $<20$ & - \\
\hline
\end{tabular}

Table 1 Distances and distance distributions (in angstroms), and populations measured using DEER between the H3 and Habc domains in syntaxin 1A (1-262). The distributions, $\sigma$, represent the SD in the width of the distribution. The percentages of spins at each distance are given in parentheses and were determined by integration of the probability distribution. 
structure and the dihedral angles $\chi 1, \chi 2$, and $\chi 3$ (Fig. 21 b) are adjusted to those expected for a helical surface site [115], the spin-spin distance for 52R1/210R1 varies from 27 to $32 \AA$ as a result of rotations about $\chi 4$ and $\chi^{5}$. Thus, the central region of the $\mathrm{H} 3$ segment assumes a configuration similar to that observed in the syntaxin1a/Munc18 crystal structure, and only a minor population of syntaxin is open. This result is similar to that seen for the 151R1/196R1 and 129R1/214R1 spin pairs. For the two spin pairs located closer to the C-terminal end of $\mathrm{H} 3$, more heterogeneity is observed. The spin pair 105R1/216R1 (Fig. 24 b and Table 1) exhibits two shorter distances where the major distance is centered at $35 \AA$, and a longer distance component at $47 \AA$, which represents $\sim 6 \%$ of the total interacting spin population. In the crystal structure of the closed state, this labeled pair is separated by $\sim 32-34 \AA$. The spin pair $105 \mathrm{R} 1 / 225 \mathrm{R} 1$ also exhibits a range of distances from 20 to $35 \AA$ (Table 1), with one minor population centered near $45 \AA$. Thus, the results of these distance measurements indicate that there is heterogeneity in the position of the $\mathrm{H} 3$ segment in the syntaxin 1a structure, particularly at the $\mathrm{C}$ terminal end; however, $\mathrm{H} 3$ remains closely associated with the Habc domain and only a minor population of $\mathrm{H} 3(\sim 10 \%)$ is significantly dissociated from the regulatory domain in the absence of Munc18.

The DEER experiment was repeated over a range of syntaxin concentrations and under conditions in which intermolecular dipolar interactions between singlelabeled syntaxin are not detected. Under low-salt conditions and at concentrations ranging from $2 \mu \mathrm{M}$ to $7 \mu \mathrm{M}$, dilution of the double-spin-labeled protein with unlabeled protein produced no significant change in the distance distribution between spins (see Fig. S4). However, under conditions that promote 
oligomerization (i.e., increasing ionic strength and protein concentration), the modulation depth in the DEER signal was effectively eliminated in the diluted system. This indicates that in the oligomerized state the interspin distances between labels on the $\mathrm{H} 3$ motif and the Habc domain are long and are effectively indistinguishable from the intermolecular background. Thus, the minor longer distances observed in Table 1 are not the result of oligomerization, but reflect an intrinsic tendency of the $\mathrm{H} 3$ motif to dissociate from the Habc domain in the syntaxin monomer. It should be noted that there is no evidence that longer distances that might appear as background are being obscured from DEER traces such as those shown in Fig. 24. The modulation depths (the amplitudes of the DEER signals once background is subtracted) are close to what would be expected if all spin pairs were included in the distribution, and the modulation depths do not change when Munc18 is bound to syntaxin, which should eliminate any significant open population (see below).

DEER experiments are typically carried out in frozen glasses, and the sample is taken to low temperature and a cryoprotectant (in this case glycerol) is added. We carried out a series of experiments to determine whether the addition of glycerol, which is an osmolyte, might have altered the result. We repeated the DEER experiment using glycerol concentrations that ranged from $5 \%$ to $50 \% \mathrm{w} / \mathrm{v}$, and at the lower concentrations used here $(5-20 \%)$ we found no effect on the distance or distribution. However, the addition of high glycerol concentrations $(50 \% \mathrm{w} / \mathrm{v})$ suppressed the minor long-distance component. This is consistent with the existence of an equilibrium between open and closed states of syntaxin [109], and the known effects of glycerol as a stabilizing osmolyte [120-122]. 
Munc18-1 abolishes the minor open form of syntaxin 1a and straightens the H3 segment.

The DEER measurements described above were repeated with the addition of Munc18-1. The results shown in Fig. $24 \mathrm{c}$ for the 52R1/210R1 and 105R1/226R1 spin-labeled pairs are typical of those obtained at most sites: the primary distance is narrowed and the minor longer distance is effectively eliminated. One exception is the 151R1/196R1 spin pair, which is more distal from the Munc18-binding region. At this site, the minor longer distance was not significantly affected by the addition of Munc18 (see Table 1). The major distances observed in the presence of Munc18 (Table 1) are consistent with the crystal structure of the syntaxin1a/Munc18 complex.

We also made one measurement between sites 196 and 228, which lies across the length of the $\mathrm{H} 3$ segment, in the absence and presence of Munc18 (Fig. 24, b and c, respectively). As can be seen in Fig. 24 b, the interspin distances obtained for 196R1/228R1 have a remarkably wide distribution, with mean distances ranging from 24 to $49 \AA$. This indicates that the H3 domain must assume some configurations that are substantially bent. These highly bent configurations could result from the fraction of protein that appears to be disordered in the continuous-wave spectra (Fig. 22). Upon addition of Munc18, the dipolar evolution produces a clear oscillation, which yields a narrower distribution with two main peaks. The longer peak at $42 \AA$ is close to the $\mathrm{C} \beta-\mathrm{C} \beta$ distance of $44 \AA$ in the crystal structure, and an examination of the likely rotameric states of R1 indicates that the two distances could correspond to two different rotamers of R1. 
The equilibrium between open and closed forms of syntaxin 1a is shifted toward the open state in membrane-associated full-length syntaxin.

In the soluble form of syntaxin without the transmembrane linker (residues 1-262), the $\mathrm{H} 3$ segment remains closely associated with the Habc domain. We examined the state of the H3 domain in full-length syntaxin 1a (residues 1-288) reconstituted into POPC/POPS (3:1) vesicles with DEER for several of the spin pairs described above. Shown in Fig. 25 a are DEER signals and distributions for 52R1/210R1. For this spin pair, $\sim 11 \%$ of syntaxin is in an open configuration in solution, and this open state increases to $\sim 40 \%$ in the full-length protein. The same trend is seen for other spin pairs where the equilibrium is shifted toward the open state by approximately a factor of 3-4. As seen for the soluble fragment, addition of Munc18 suppresses the long-distance component and narrows the distance distribution (Fig. 25 b). It should be noted that on the membrane interface we do not detect dipolar interactions between single spin-labeled syntaxin (indicating oligomerization); thus, the more-open state on these bilayers is not driven by oligomerization. In addition to POPC/POPS, full-length protein was also reconstituted into bilayers of pure POPC. The result obtained in POPC is identical to that obtained in POPC/POPS, indicating that the presence of acidic lipid does not play a role in stabilizing the more-open conformation.

Deletion of an N-terminal segment of syntaxin 1a has minor effects on the ability of Munc18 to close the syntaxin 1a structure.

Measurements obtained using diffraction show that Munc18 fails to close the soluble syntaxin 1a fragment (1-262) but will close a syntaxin fragment lacking an 
a
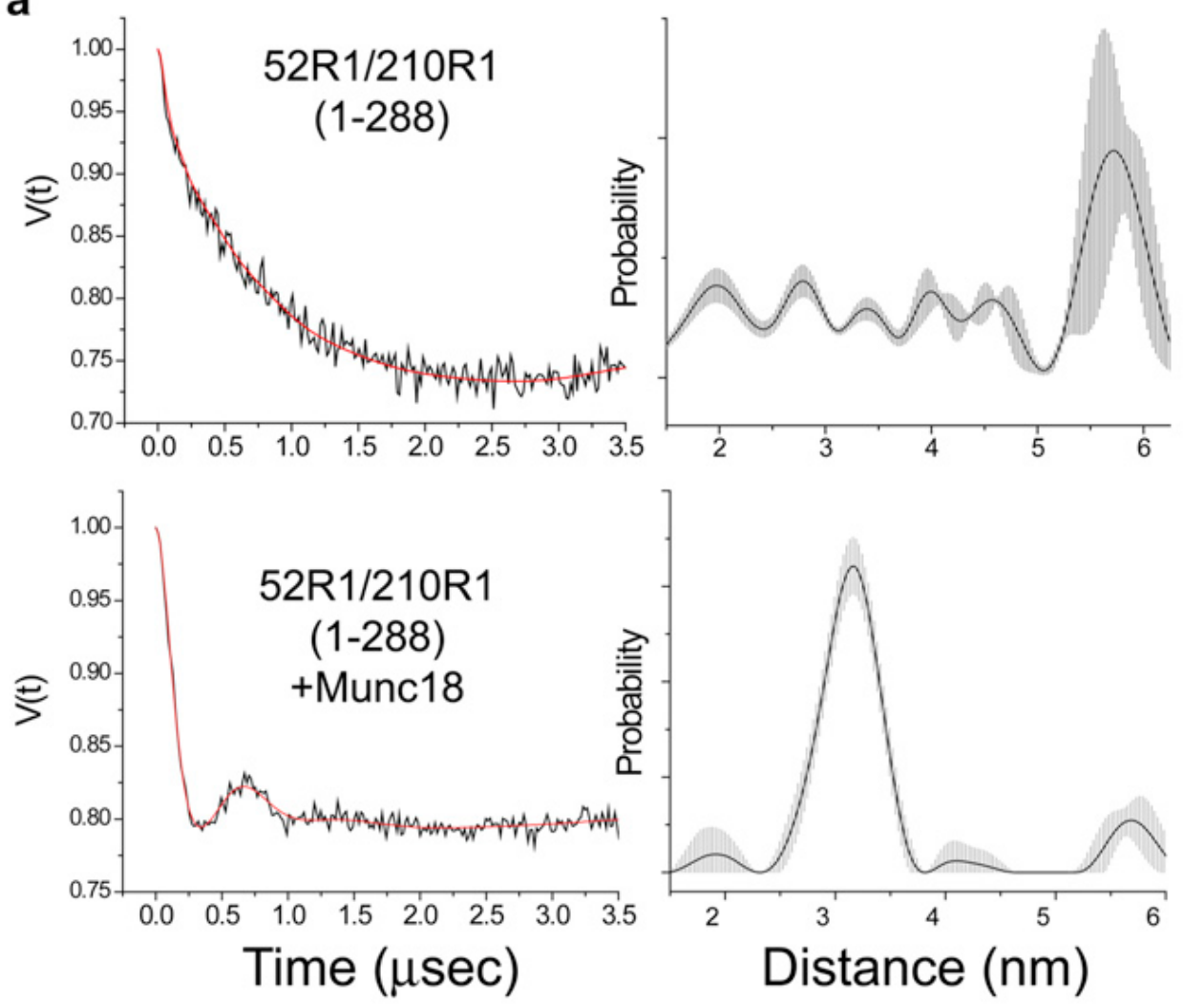

b
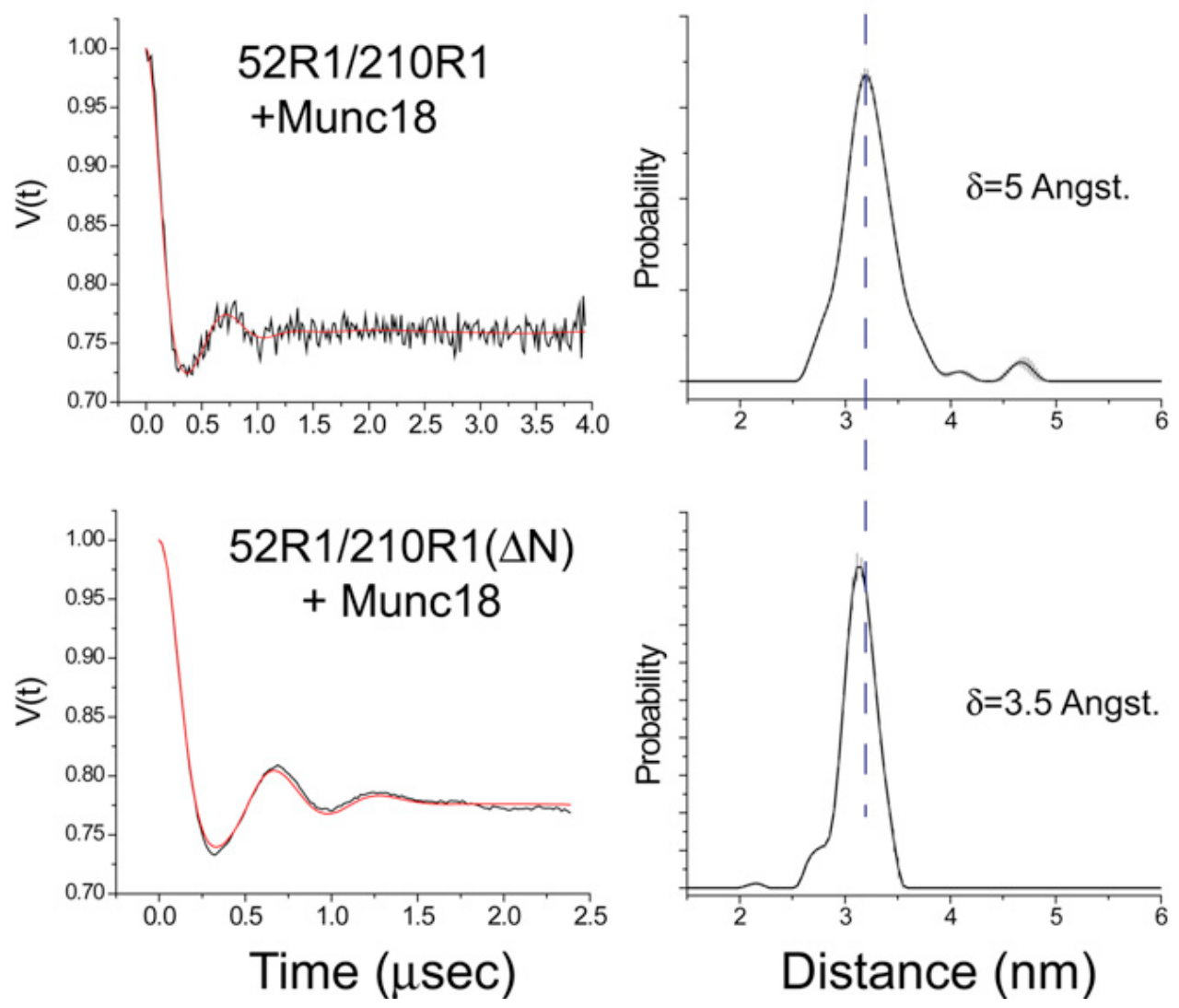
Figure 25 (a) DEER data (left panel) and corresponding distance distributions (right panel) for the spin pair 52R1/210R1 in full-length syntaxin 1a (1-288) that is reconstituted into POPC/POPS (3:1) lipid vesicles at a protein/lipid molar ratio of 1:1000. Data were obtained in the absence (upper panel) presence (lower panel) of Munc18-1. Syntaxin (1-288) was used at a concentration of $100 \mu \mathrm{M}$ in $140 \mathrm{mM}$ $\mathrm{NaCl}, 2.7 \mathrm{mM} \mathrm{KCl}, 12 \mathrm{mM}$ phosphate buffer, $\mathrm{pH}$ 7.3. (b) DEER data and distributions for $52 \mathrm{R} 1 / 210 \mathrm{R} 1$ in soluble syntaxin that includes the $\mathrm{N}$-terminal segment (1-262, upper panel) or has the N-terminal segment deleted (27-262 or DN, lower panel). The half-maximal widths of each distribution are shown. The shaded regions in the probability distributions represent the range of solutions that can be achieved by variation of background subtraction (due to intermolecular spin interactions). Syntaxin (1-262) was used at a concentration of $40 \mu \mathrm{M}$ in $140 \mathrm{mM}$ $\mathrm{NaCl}, 2.7 \mathrm{mM} \mathrm{KCl}, 12 \mathrm{mM}$ phosphate buffer, $\mathrm{pH}$ 7.3. The solid (or red) traces on the DEER data are the fits that yield the corresponding distributions. 
N-terminal segment. To determine whether this segment alters the ability of Munc18 to produce a closed state in syntaxin, we performed DEER measurements across the H3 and Habc domains in syntaxin 27-262 and compared them with measurements for the intact soluble syntaxin fragment (1-262). The comparison in distributions between soluble syntaxin with and without the N-terminal 26 residues for $52 \mathrm{R} 1 / 210 \mathrm{R} 1$ is shown in Fig. 25 b. As can be seen in this figure, the two distributions obtained for the syntaxin1a/Munc18-1 complex are very similar, with nearly identical mean distances. Removal of the N-terminal segment produces a small but significant narrowing of the distance distribution, decreasing the width of the distribution from 5 to $3.5 \AA$. This narrowing of the distribution can be seen in the primary DEER data as an additional oscillation in the dipolar evolution.

\section{Structure of the open form of syntaxin 1a determined using EPR-derived distance constraints and simulated annealing.}

As indicated above, the dominant mean short distances determined by DEER are consistent with the crystal structure of the syntaxin 1a/Munc18-1 complex. To generate an approximate model for the open state of syntaxin 1a, we took the minor long distances in Table 1 and used them as constraints in a series of simulated annealing trials using Xplor-NIH, where the spin-labeled side chain R1 was modeled into appropriate sites in the syntaxin 1a structure. Although the H3 segment is more disordered in the absence than in the presence of Munc18, we held H3 in a helical configuration consistent with the syntaxin/Munc18 crystal structure and allowed the linker connecting $\mathrm{H} 3$ to the Habc domain to be flexible (residues 157-189). The details of the simulated annealing are described in Materials and 
Methods. Shown in Fig. 26 are the five lowest-energy structures obtained by simulated annealing, where each structure is aligned to the Habc domain. The EPRderived distance restraints are satisfied in each of these structures. In this open structure, the backbone atoms in the $\mathrm{H} 3$ domain are positioned $\sim 35 \AA$ from the Habc domain, and the $\mathrm{H} 3$ domain in these structures is splayed over an arc on one side of the Habc domain. It should be noted that the H3 segment is likely to be more much disordered than the representation shown in Fig. 26. In addition, variability in the linker joining $\mathrm{H} 3$ and $\mathrm{Habc}$ may be due in part to the fact that there are no restraints for this region, which is underdetermined. 

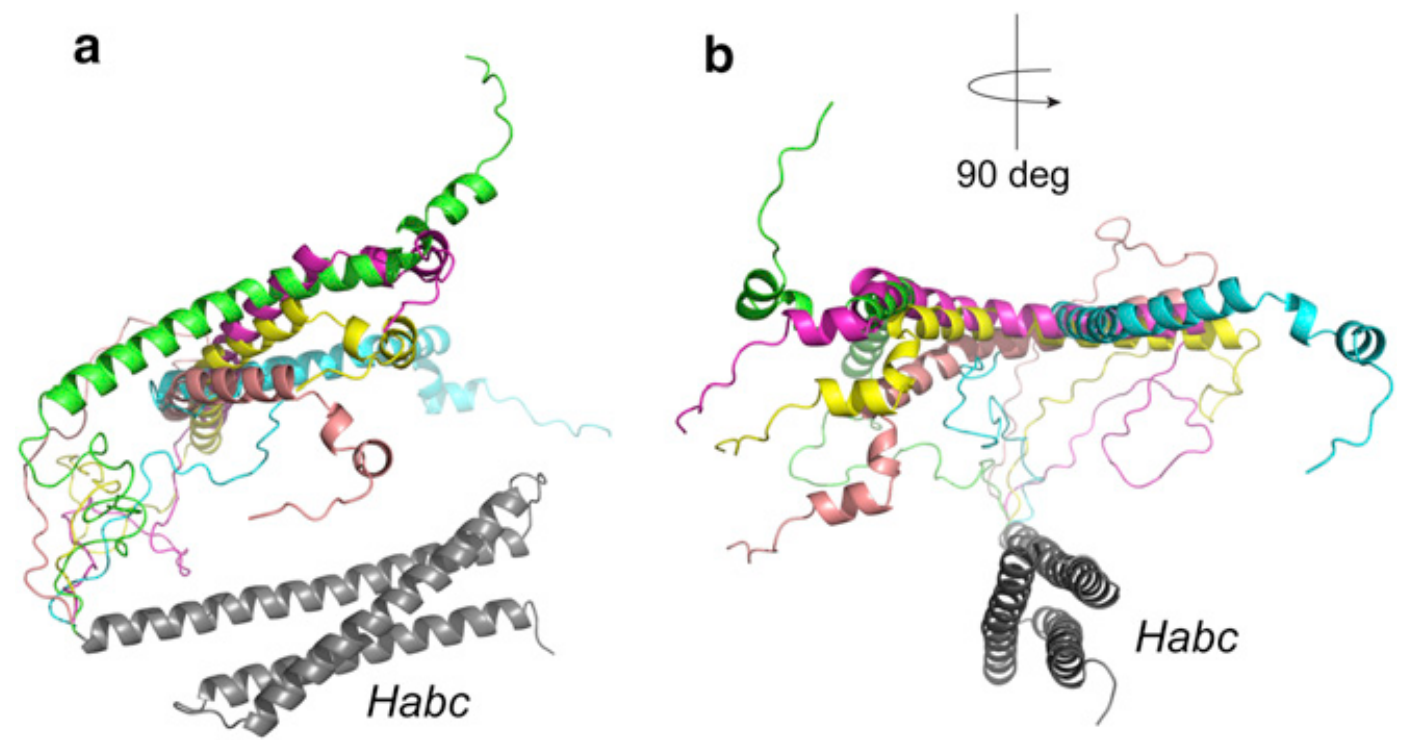

Figure 26 Model for the open state of syntaxin 1a (1-266) obtained by simulated annealing using the long-range distance restraints obtained by DEER (see Table 1). (a and b) The five lowest-energy structures are shown, with views rotated by $90^{\circ}$. 


\subsection{DISCUSSION}

The data presented here reveal the dynamics and conformations of syntaxin 1a in the presence and absence of Munc18-1. EPR spectroscopy indicates that the H3 segment of syntaxin is in conformational exchange between ordered and disordered states, each of which has a significant population. As a result, the $\mathrm{H} 3$ segment is neither a single uniform helical segment nor a completely disordered segment in the presence of the Habc domain. When distances are measured across the H3 and Habc regions in syntaxin 1a (1-262), the central portion of $\mathrm{H} 3$ is closely associated with the Habc domain and the configuration of syntaxin is predominantly closed.

Assembly of the SNARE complex is generally believed to occur in an N- to C-terminal direction along the SNARE motif [31], and the closed state of syntaxin is thought to inhibit assembly because the H3 segment is interacting with the Habc domain and is not available to interact with the other SNARE partners, such as SNAP25 and synaptobrevin. The data presented here indicate that both local fluctuations along $\mathrm{H} 3$ and an open-closed equilibrium take place. Local fluctuations along H3, especially near the N-terminal end of the H3 segment, may help promote interactions with other SNAREs and will occur even though the structure is approximately closed. In solution, assembly of the SNARE complex is inhibited by Munc18-1 [63], and this may be due in part to increased order along the H3 segment, which limits encounters between the N-terminal end of the $\mathrm{H} 3$ motif and the other SNARE partners. The limitation or regulation of dynamic transitions at the Nterminal end of syntaxin is likely to be an important regulator of SNARE assembly, and will modify encounters between syntaxin and other SNARE partners [106, 107]. The effect of Munc18 resembles that of other allosteric processes that control protein conformational equilibria and appear to be important in regulating protein- 
protein interactions.

As shown in Table 1 and Fig. 24, the EPR data indicate that syntaxin (1-262) is largely in a closed configuration. This finding and the observation that the H3 domain is more disordered toward its C-terminus are consistent with a previous NMR study [4], but they present a somewhat different picture than that obtained in a previous study using fluorescence [40], in which the majority of the H3 domain was found to be dissociated from the Habc domain. The reasons for the differences between our results and the earlier fluorescence result are not entirely clear, but the open and closed states of syntaxin are in equilibrium and must be relatively close in energy. As a result, relatively minor shifts in energy, perhaps due to experimental conditions, different protein constructs, or probe incorporation, may have altered the open/closed populations.

The EPR data indicate that Munc18 binding has two effects: it shifts the conformational equilibria along H3 toward the more ordered state, and it suppresses the open configuration of syntaxin. Structural restraints obtained from EPR in the presence of Munc18 are consistent with the closed state defined by the crystal structure $[43,63]$, and with the results obtained from single-molecule fluorescence resonance energy transfer and NMR $[4,40]$. These results contrast with recent work using cross-linking and solution scattering, which indicated that syntaxin 1a does not assume a closed configuration upon Munc18-1 binding unless an N-terminal syntaxin peptide is removed [105]. The source of these differences is not clear. Measurements made here in the presence and absence of the N-terminal peptide (Fig. 25 b) indicate that the only difference is a narrowing in the distance distribution measured 
between the $\mathrm{H} 3$ and Habc domains, but both forms (with or without the N-terminal segment) are dominated by a mean distance corresponding to the closed form in the presence of Munc18. In the previous scattering study [105], Munc18 was prepared using a baculovirus-infected insect cell line rather than a bacterial expression system, and conceivably differences in the protein preparation used could explain some of the discrepancies.

As shown in Fig. 25 a, the fraction of open syntaxin is enhanced in the fulllength membrane-associated protein. In full-length protein, Munc18 produces a dramatic shift in populations from an open to a closed configuration. The source of this difference when compared with the soluble form is not entirely clear, but transient interactions of the $\mathrm{H} 3$ domain with the membrane or steric interference due to the membrane interface might alter the open-closed equilibrium in syntaxin. The juxta-membrane region of syntaxin is highly basic and is found to associate with the membrane interface [123]; however, electrostatic interactions with the protein interface require acidic lipid, which does not appear to be playing a role in promoting the open state. The greater fraction of open state in full-length syntaxin might result from oligomerization of the $\mathrm{H} 3$ segment due to an increased local concentration of syntaxin on the membrane interface; however, we did not find evidence for enhanced aggregation on the membrane surface at the lipid/protein ratios used here. On the bilayer, the open state is increased by a factor of $\sim 3$, and for $52 \mathrm{R} 1 / 210 \mathrm{R} 1$ the conformational energy difference between solution and bilayer is estimated to be $0.8-0.9 \mathrm{kcal} / \mathrm{mole}$. These results indicate that measurements of syntaxin dynamics and conformational equilibria, which are likely critical for directing assembly of the SNARE complex, are highly context dependent, and that 
measurements in a native bilayer environment may be necessary to establish the equilibria and interactions that are important for neuronal fusion.

In summary, the $\mathrm{H} 3$ or SNARE-forming segment of syntaxin 1a is in equilibrium between ordered and disordered forms, and the $\mathrm{H} 3$ segment remains closely associated with the Habc domain in the soluble syntaxin construct. A minor open population is observed where the $\mathrm{H} 3$ domain is dissociated from the Habc domain by $\sim 35 \AA$. This open form is more highly populated in full-length syntaxin (1-288) reconstituted into lipid bilayers. An additional open form, where the H3 domain is displaced from the Habc domain by $>60 \AA$, is present when syntaxin 1a is oligomerized. Munc18 binding promotes the closed state of syntaxin and produces a shift to a more ordered, structured state along H3. The structural fluctuations along H3, which are modulated by Munc18, are likely a key dynamic feature that controls assembly of syntaxin into the SNARE complex.

\subsection{ACKNOWLEDGMENTS}

We thank Dr. Jeffrey Ellena of the Biomolecular NMR Facility, University of Virginia, for help with the TRACT measurements. This work was supported by a grant from the National Institutes of Health (NIGMS GM072694). 


\subsection{SUPPORTING MATERIAL}

\section{Characterization of syntaxin aggregation (1-262)}

The soluble syntaxin construct (1-262) was examined using several approaches to characterize its state of aggregation under conditions of varied concentration and ionic strength. Shown in Fig. S1a are examples of ${ }^{15} \mathrm{~N}$ TRACT NMR data obtained on syntaxin (1-262) using an approach described previously [118]. This experiment was performed on $200 \mu \mathrm{M}$ of the ${ }^{15} \mathrm{~N}$ labeled $\mathrm{H} 3 / \mathrm{Habc}$ fragment in $20 \mathrm{mM}$ phosphate buffer, $\mathrm{pH}=7.3$, using a Bruker Avance III 600 NMR spectrometer and 25 to 100 evenly spaced decay times between 8 and $600 \mathrm{~ms}$. Signals were obtained by integrating the peak intensities of the backbone amide ${ }^{1} \mathrm{H}$ region (7.9 to 8.8 ppm). Both fast and slow decay curves were obtained corresponding to the two ${ }^{15} \mathrm{~N}$ transitions. Each of these curve were fit best to two rates and the results of multiple measurements yielded correlation times of $18.8( \pm 2.3)$ and $4.3( \pm 0.2)$ ns that were approximately equally populated. The longer correlation time is consistent with an apparent hydrodynamic radius near 28 Angstroms, close to that expected for a rod shaped molecule the size of syntaxin with an axial ratio of 2.5 [125]. Since a large fraction of the syntaxin (1-262) amide backbone has significant flexibility, the short correlation time is likely to be due to this portion of the protein. Shown in Fig. S1b are DLS measurements on syntaxin 210R1, which yielded a similar particle radius of about $3 \mathrm{~nm}$.

Pulse EPR measurements and DEER were made on single labeled syntaxin mutants to determine whether these spin labeled mutants aggregated. The phase memory times in all our samples ranged between 2.5 and $3 \mu$ s, indicating that extensive non- 
specific aggregation is not taking place under the conditions used here. Much shorter phase memory times (less than $1 \mu \mathrm{s}$ ) are observed for syntaxin samples that aggregate. Shown in Fig. S1c are DEER traces for 210R1, which show weak dipolar coupling at low to moderate ionic strength but stronger dipolar coupling at high ionic strength. These data are consistent with the oligomerization of syntaxin (with both an $\mathrm{H} 3$ and Habc domain), which has been previously reported based upon analytical ultracentrifugation [38] and NMR [41]. The modulation depth of the DEER signal is dependent upon the number of excited spin-paris and this signal may be used to obtain an estimate of the extent of dimerization as described previously [119]. Under the conditions shown in Fig. S1c, less than $10 \%$ of the protein is dimerized at $150 \mathrm{mM} \mathrm{NaCl}$, and approximately $40 \%$ dimerized at 300 $\mathrm{mM} \mathrm{NaCl}$. These fractions varied between different R1 mutants, but under low ionic strength conditions, oligomerized syntaxin ranged from 5 to $15 \%$. 
a

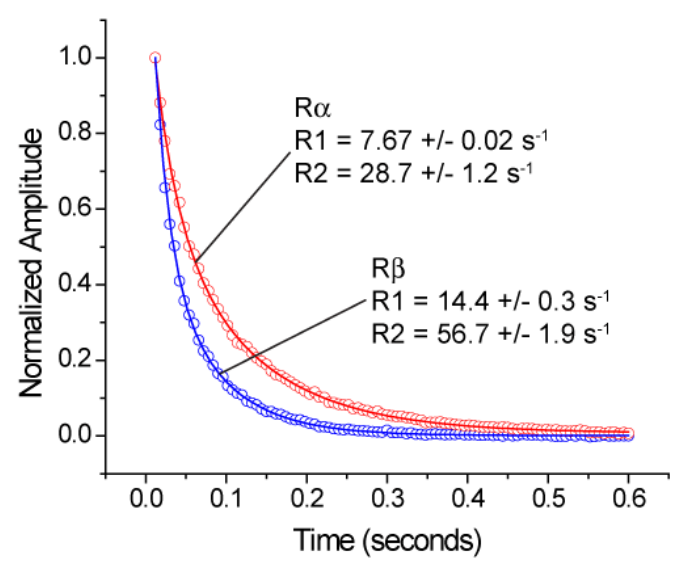

C

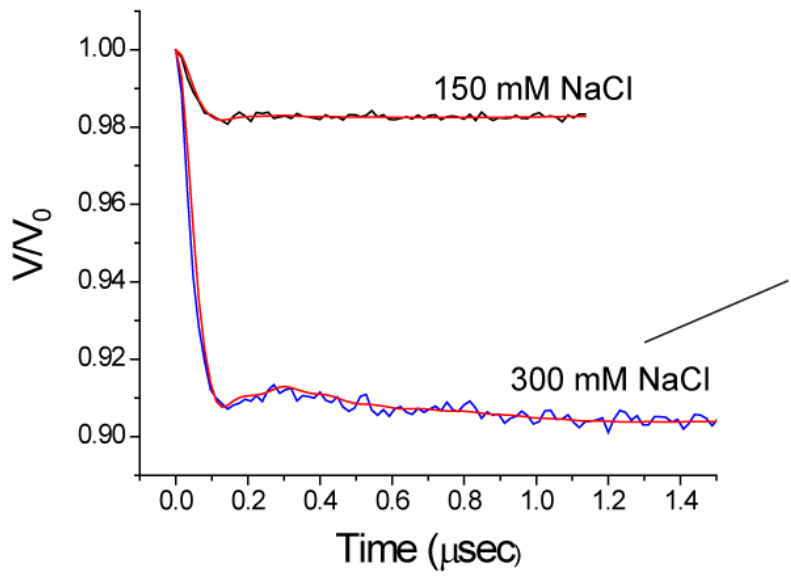

b
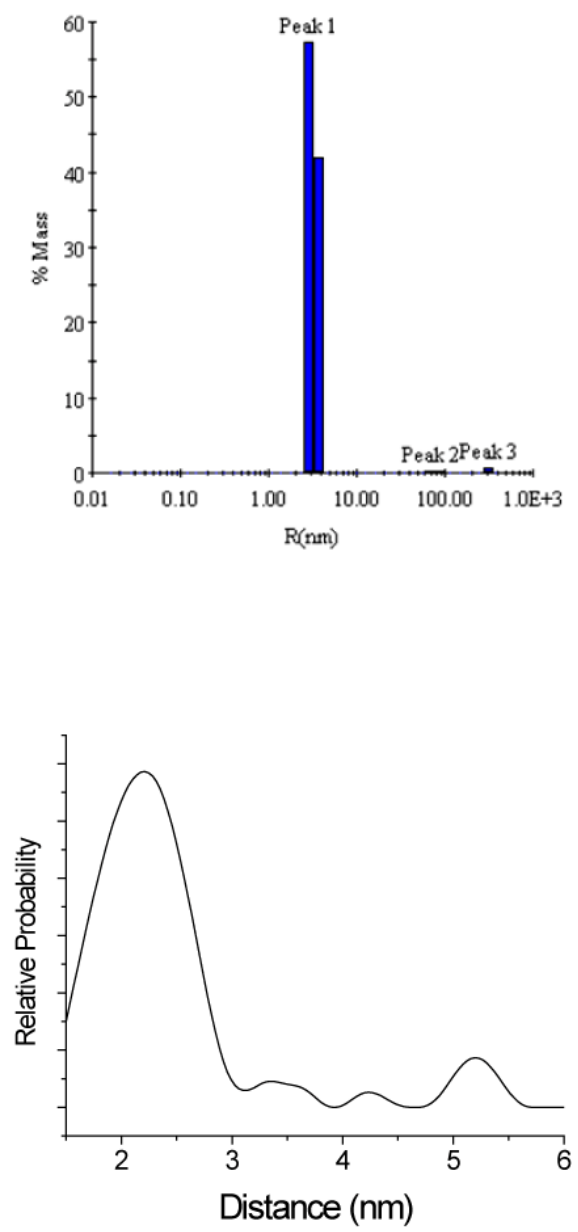

Figure S1. (a) TRACT NMR data from ${ }^{15} \mathrm{~N}$-labeled syntaxin (1-262) obtained as described above in $20 \mathrm{mM}$ phosphate buffer, $\mathrm{pH}=7.3$. The long correlation time is consistent with the effective hydrodynamic radius of the syntaxin monomer. (b) DLS measurements on syt (1-266) 210R1 yield a single peak with a particle size of about $3 \mathrm{~nm}$. The samples contained $60 \mu \mathrm{M}$ protein in phosphate buffered saline (140 mM NaCl, $2.7 \mathrm{mM} \mathrm{KCl,} 10 \mathrm{mM} \mathrm{Na}_{2} \mathrm{HPO}_{4}, 1.8 \mathrm{mM} \mathrm{K}_{2} \mathrm{HPO}_{4}, \mathrm{pH}=7.3$ ) and were made using a miniDAWN multi-angle light scattering instrument (Wyatt 
Technologies, Santa Barbara, CA). (c) DEER measurements made on a $40 \mu \mathrm{M}$ singly labeled syntaxin mutant $210 \mathrm{R} 1$ in $10 \mathrm{mM}$ phosphate buffer $(\mathrm{pH}=7.3)$ and at the indicated concentration of $\mathrm{NaCl}$. Assuming 100\% labeling efficiency we estimate that at $150 \mathrm{mM} \mathrm{NaCl}$ approximately $8 \%$ or less of the syntaxin is dimerized. At $300 \mathrm{mM} \mathrm{NaCl}$, the fraction dimerized increases to about $40 \%$. The distribution shows that the dominant distance obtained at under conditions of 300 $\mathrm{mM} \mathrm{NaCl}$ is approximately 22 Angstroms. 


\section{Simulations of EPR spectra}

EPR spectra were simulated using the MOMD model [79] as implemented in a LabView program "Multicomponent EPR fitting" obtained from Dr. Christian Altenbach (UCLA). The general fitting procedure followed those described elsewhere [126]. Shown in Fig. S2 are examples of fits to two sites on syntaxin (1262), (a) 210R1 and (b) 167R1. Also shown are the individual simulated components. In these two cases, the principle values of the hyperfine tensor were taken as $A x x=6.2, A y y=5.0$ and $A z z=36.5$, and g-tensor values were $\mathrm{gxx}=2.0076$, gyy $=2.0050$ and $g z z=2.0023$. For both $210 \mathrm{R} 1$ and $167 \mathrm{R} 1$, the fast component was simulated using isotropic motion with correlation times of 0.6 and 0.9 ns, respectively. In these fits, the slow motional component was fit with correlation times of 2.5 and $2.3 \mathrm{~ns}$ and order parameters of 0.25 and 1.1, respectively. The tilt angle between the diffusion tensor and the magnetic reference frame was taken as that defined previously for labels on exposed surface sites [126]. It both cases, the less mobile component resembles those observed for labels on dynamic helix surface sites (8). Smaller order parameters could be exchanged for slower motional rates while still producing simulations with acceptable fits to the spectra; therefore, these motional parameters are not uniquely determined in these simulations. Populations of components in the spectra were determined by integration of the spectral components, which is provided as an output in the program. The fast component represented approximately $35 \%$ and $20 \%$ of the signal for $210 \mathrm{R} 1$ and 167R1, respectively. Populations could also be determined by simulation and direct subtraction of the mobile isotropic component in the EPR spectrum. Either approach gave similar results. 

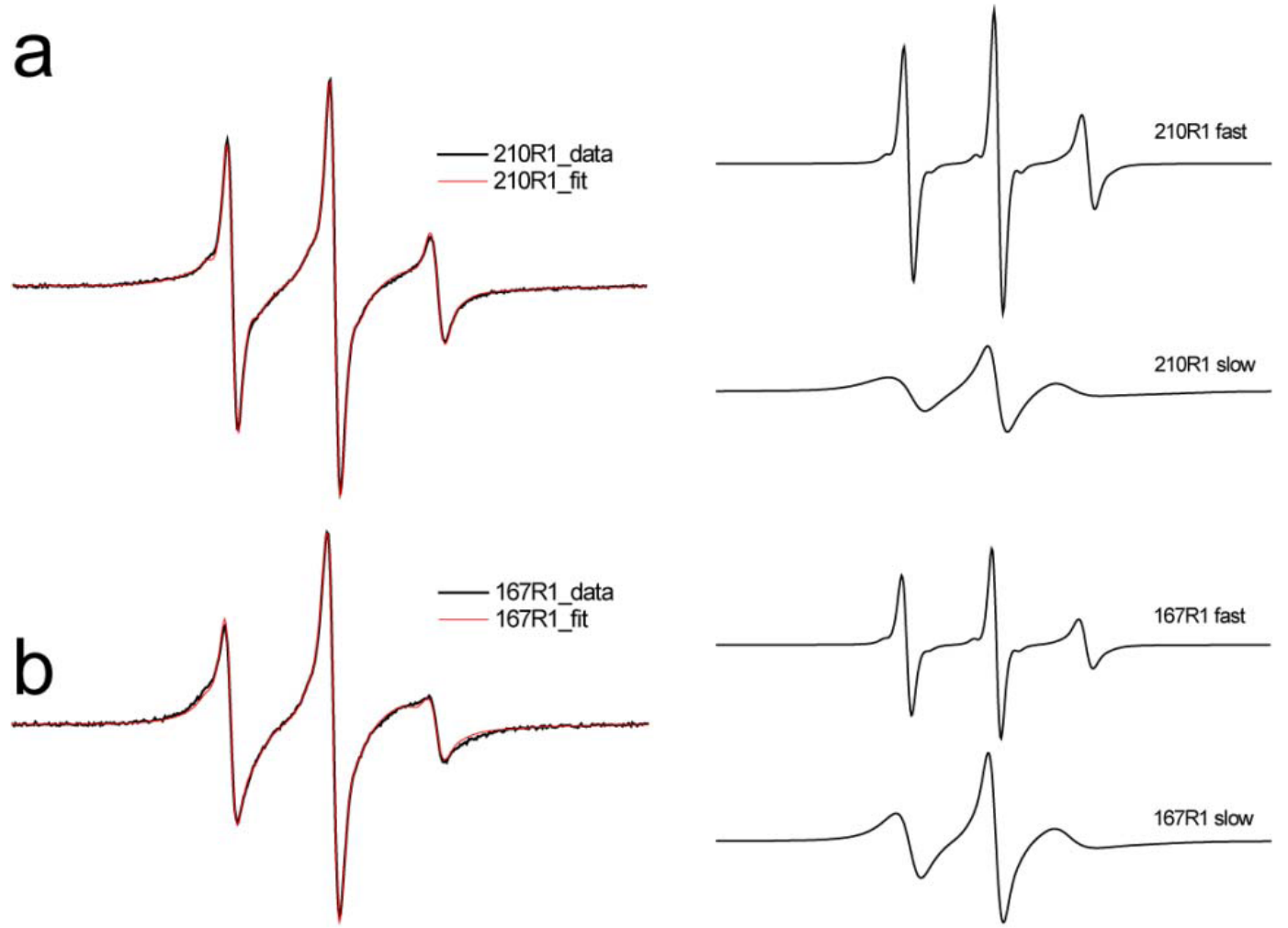

Figure S2. (a) Experimental (black trace) and simulated spectra (red trace) for 210R1 and (b) 167R1. The spectra on the right display the individual motional components of the simulated spectra. 
Titration of the EPR spectra of single syntaxin mutants with sucrose.

Sucrose was used to determine whether the motional components seen in the EPR spectra shown in Fig. 22 were the result of rotameric states of the spin label or conformational states in syntaxin (see main text). Shown below in Fig. S4a is a titration of label 192R 1 ranging from 0 to $40 \% \mathrm{w} / \mathrm{v}$ sucrose. This titration was carried out for this and 4 additional sites along the H3 segment and the data are plotted assuming a simple two-state transition between ordered (folded) and distordered (unfolded) states in H3. Two sets of data are included for syntaxin immobilized on resin though the N-terminal $\mathrm{His}_{6}$ tag. These data demonstrate that sucrose induced changes are not the result of sucrose-induced aggregation. 

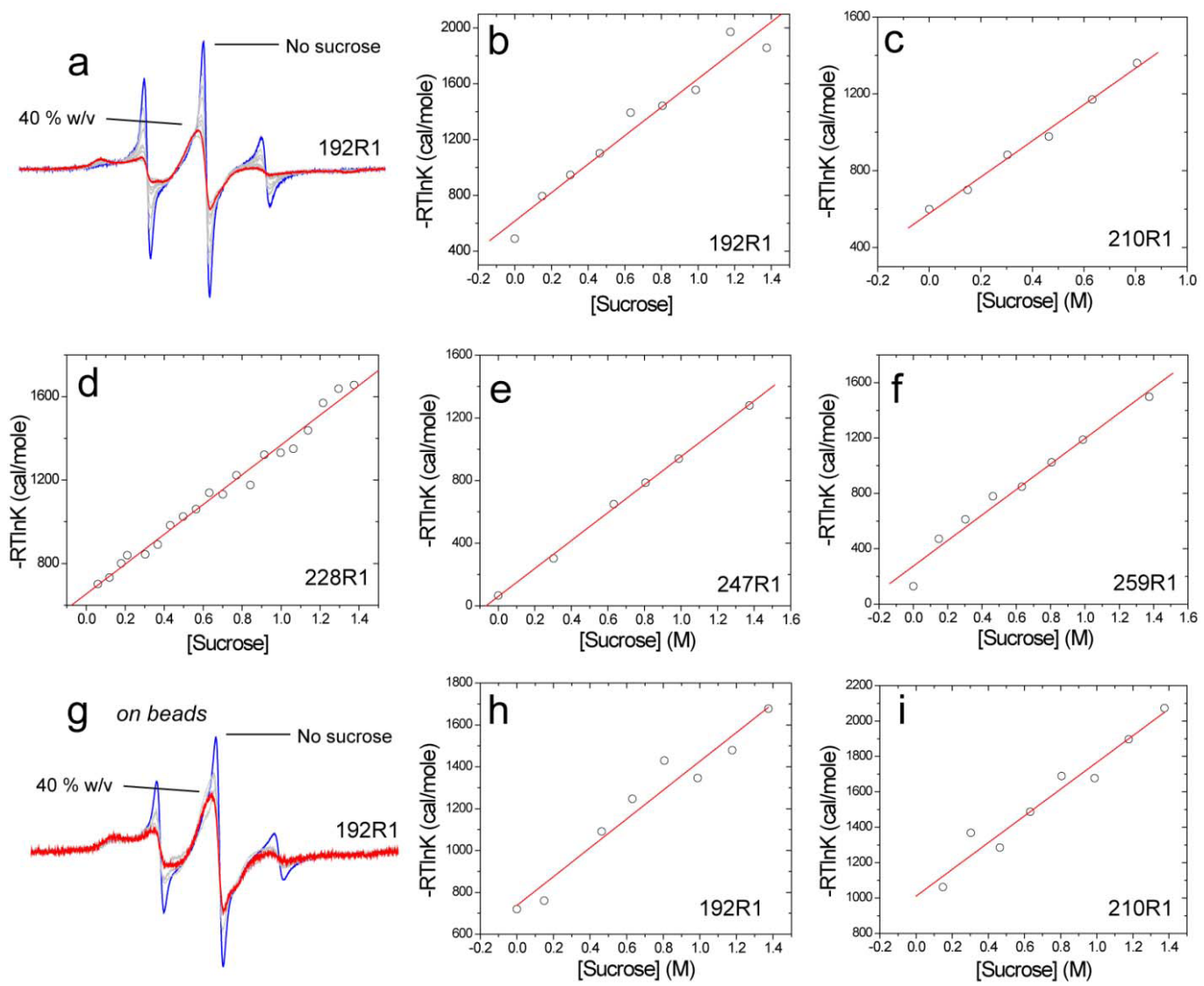

Figure S3. Titration of the EPR spectra for five sites along the H3 domain of syntain as a function of sucrose concentration. (a) EPR spectra for 192R1 at concentrations of sucrose ranging from 0 to $40 \% \mathrm{w} / \mathrm{v}$. Plots of the free energy for the folded/unfolded equilibrium as a function of sucrose concentration (molar) for (b) site 192R1, (c) 210R1, (d) 228R1, (e) 247R1 and (f) 259R1. The free energy change per molar of sucrose concentration was determined using a linear fit for data in (b) through (f) and yield values of $1.02 \pm 0.08,0.94 \pm 0.04,0.72 \pm 0.02,0.92 \pm$ $0.06,0.89 \pm 0.02 \mathrm{kcal} / \mathrm{mole}$, respectively. Titrations have also been carried out for syntaxin (1-262) immobilized on beads using the His-tag. In (g) are EPR spectra for 192R1 at sucrose concentrations from 0 to $40 \% \mathrm{w} / \mathrm{v}$, and in (h) and (i) are free 
energy plots as a function of sucrose concentration for 192R1 and 210R1, respectively. On beads, the equilibrium of $\mathrm{H} 3$ is shifted slightly towards the more ordered state, and on beads, the free energy for the folded/unfolded equilibrium as a function of sucrose concentration (molar) for sites 192R1 and 210R1 was $0.690 \pm$ 0.07 and $0.760 \pm 0.07 \mathrm{kcal} / \mathrm{mole}$. 
Inter- versus intra-molecular spin interactions in oligomerized syntaxin (1262).

As shown in Fig. S4, when syntaxin is oligomerized but diluted with unlabeled wild-type protein, dipolar interactions between spins on the $\mathrm{H} 3$ domain and the Habc domain are not detected. Because intramolecular interactions between the H3 and Habc domains are not detected, these domains must be displaced by a significant distance in the aggregated state. As shown in Fig. S5, additional distances appear in the aggregated state, which must be due to intermolecular spin interactions. These may be reversed by dilution of the protein or by the binding of Munc18. 


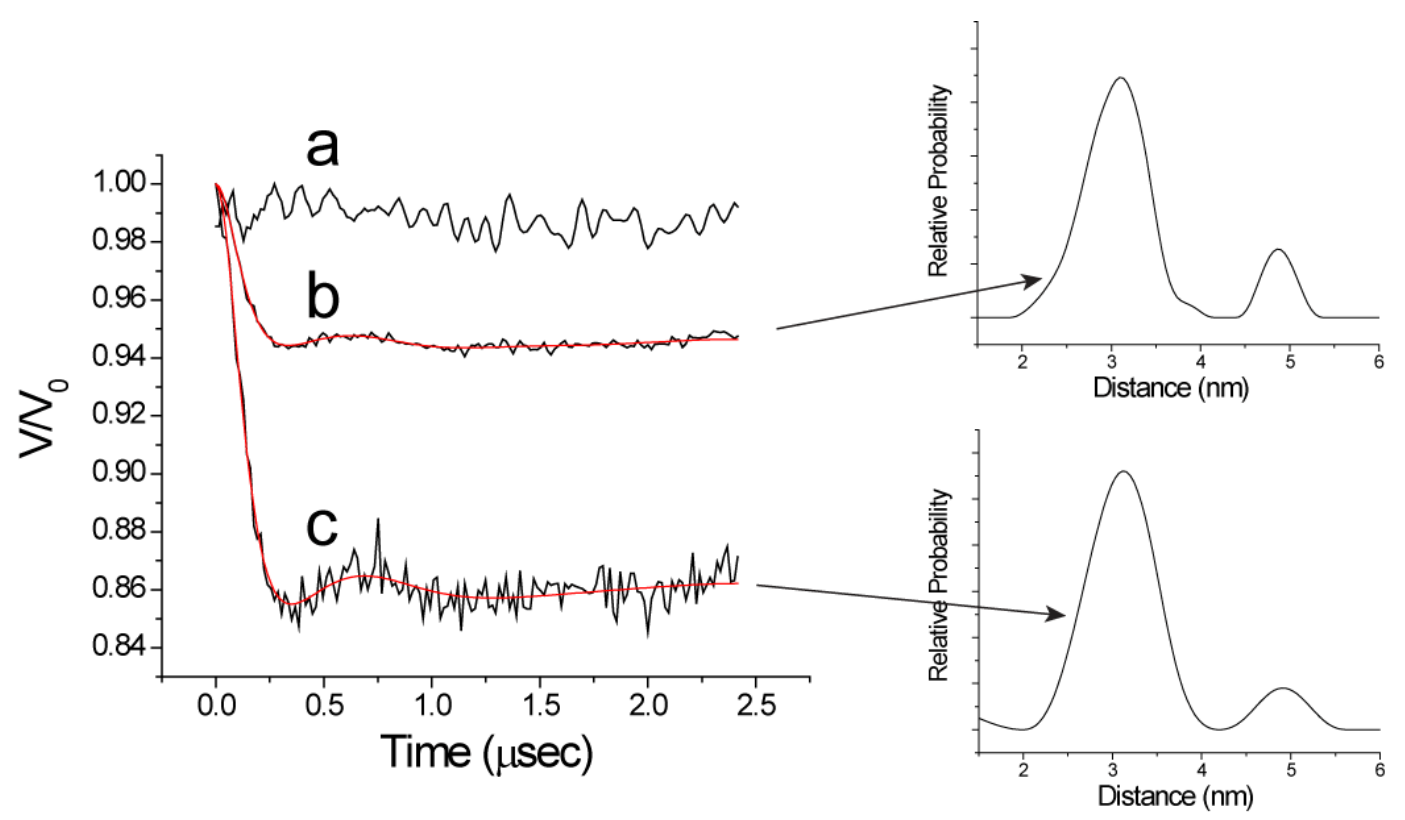

Figure S4. DEER signals obtained from syntaxin (1-262) 52R1/210R1 when diluted with a 5-fold excess of wild type protein in under conditions where (a) significant aggregation is expected $(30 \mu \mathrm{M}$ protein, ionic strength $>300 \mathrm{mM})$, (b) some aggregation is expected $(7 \mu \mathrm{M}$ protein, ionic strength $=150 \mathrm{mM})$, and $(\mathbf{c})$ no aggregation is expected $(2 \mu \mathrm{M}$ protein with $20 \mathrm{mM}$ MOPS, $\mathrm{pH}=7.3)$. For $(\mathrm{b})$ and (c) the corresponding distance distributions (right panels) and fits (red traces) are shown for the data. The greater noise level in (a) is due to a much shorter acquisition time and not to differences in the phase memory times, which ranged from 3 to $4 \mu \mathrm{sec}$ for these samples. 


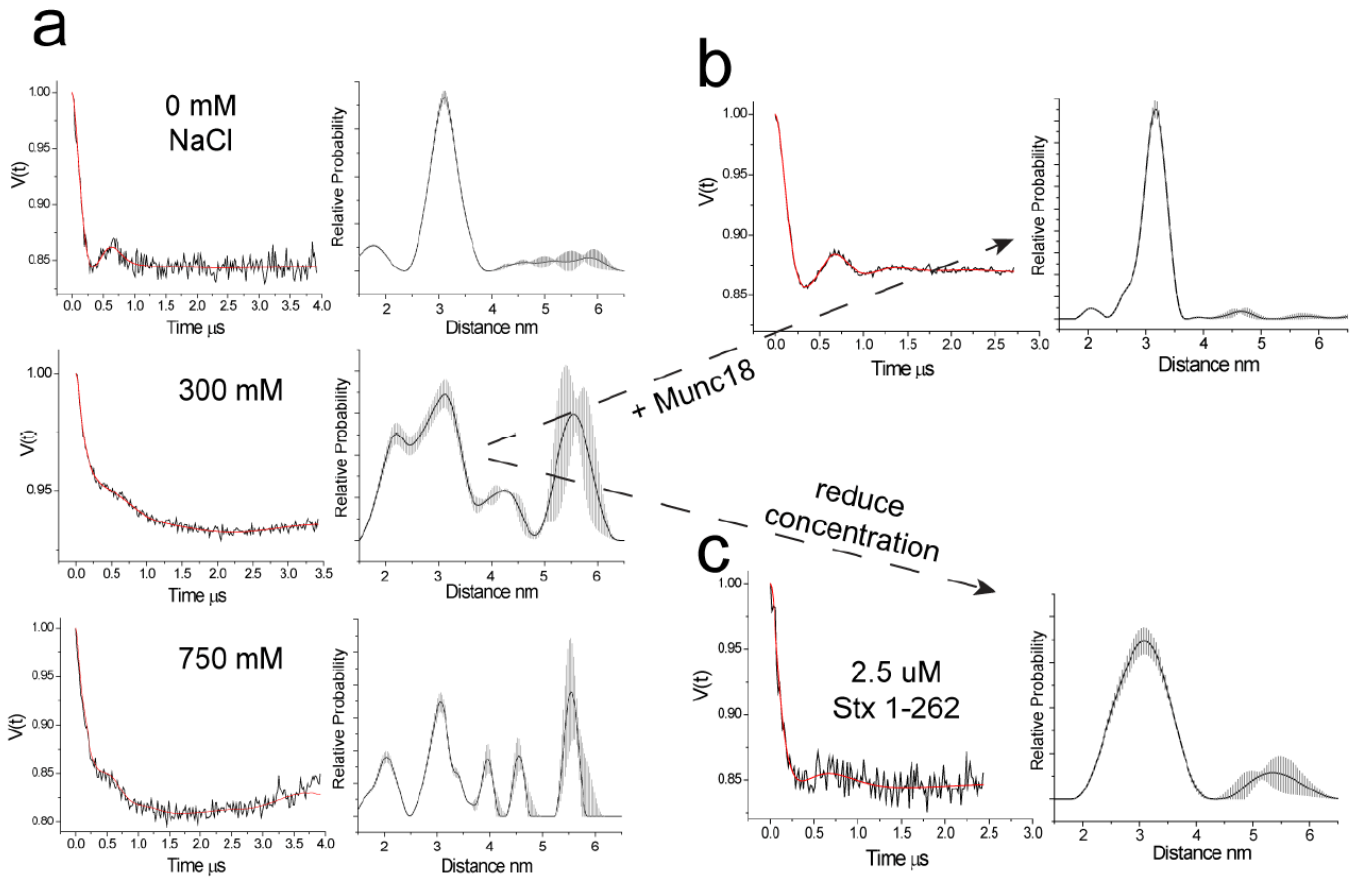

Figure S5. DEER signals and distributions measured for $52 \mathrm{R} 1 / 210 \mathrm{R} 1$ in the soluble syntaxin 1a construct (1-262). Both ionic strength and protein concentration appear to modulate the distribution of distances between $\mathrm{H} 3$ and Habc, but as shown in Fig. S4, these additional signals are intermolecular and are the result of oligomerization. In (a) syntaxin $52 \mathrm{R} 1 / 210 \mathrm{R} 1$ is at a concentration of $40 \mu \mathrm{M}$ and increasing the ionic strength (from $10 \mathrm{mM}$ buffer to buffer plus $750 \mathrm{mM} \mathrm{NaCl}$ ) increases the fraction of longer distances and results in the appearance of a shorter distance component. For the sample containing $300 \mathrm{mM}$ salt (middle panel in (a)), the closed state may be stabilized either by the addition of Munc18-1 (b) or dilution of protein sample to $2.5 \mu \mathrm{M}$ (c). In (c) a shorter evolution time was used than at the higher concentrations, but the phase memory times increase and are greater than 4 $\mu$ sec. 
Chapter 4: The SNARE Motif of Synaptobrevin

Exhibits an Aqueous-Interfacial Partitioning That Is Modulated by Membrane Curvature 

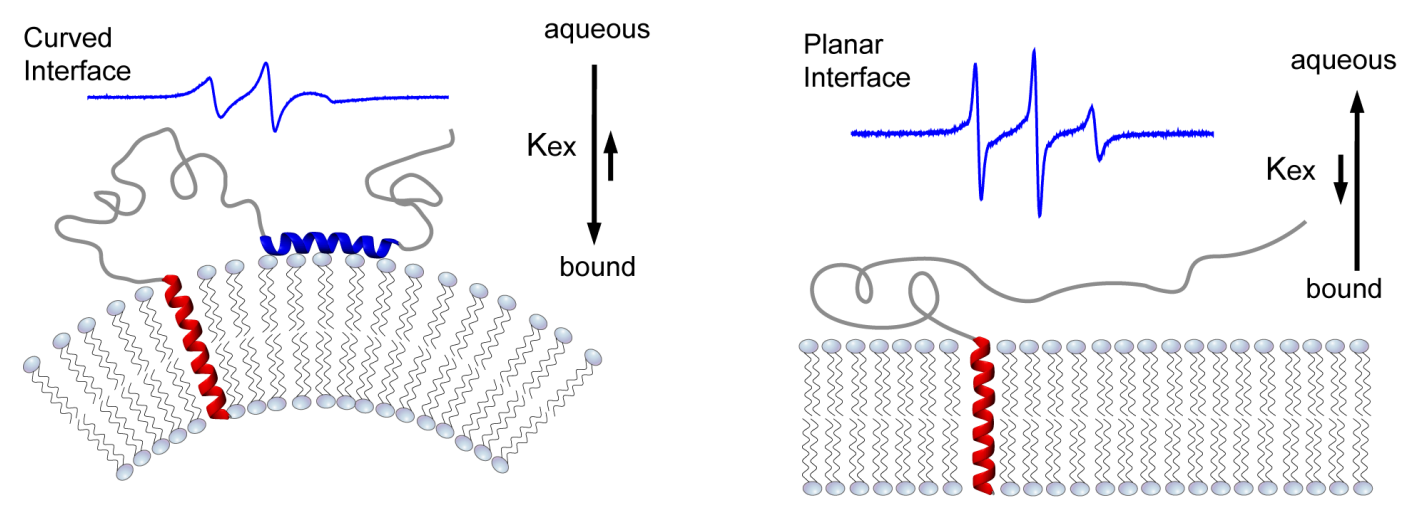

4.1 ABSTRACT: The structure and interfacial association of the full-length vesicle SNARE, synaptobrevin, were compared in four different lipid environments using nuclear magnetic resonance and electron paramagnetic resonance spectroscopy. In micelles, segments of the SNARE motif are helical and associated with the interface. However, the fraction of helix and interfacial association decreases as synaptobrevin is moved from micelle to bicelle to bilayer environments, indicating that the tendency toward interfacial association is sensitive to membrane curvature. In bilayers, the SNARE motif of synaptobrevin transiently associates with the lipid interface, and regions that are helical in micelles are in conformational and environmental exchange in bicelles and bilayers. This work demonstrates that the SNARE motif of synaptobrevin has a significant propensity to form a helix and exchange with the membrane interface prior to SNARE assembly. This transient interfacial association and its sensitivity to membrane curvature are likely to play a role in SNARE recognition events that regulate membrane fusion. 


\subsection{INTRODUCTION}

Neurotransmitter release results from a membrane fusion event that joins the synaptic vesicle membrane with the presynaptic plasma membrane. This fusion event is driven by soluble N-ethylmaleimide-sensitive factor attachment receptor proteins (SNAREs), which form the core of the membrane fusion machinery. SNAREs assemble into a tight four-helix bundle that is thought to provide the energy required to overcome the barrier to fusion, $[31,101]$ and in the neuronal system, the SNARE complex is formed from syntaxin 1a and SNAP-25 on the plasma membrane and synaptobrevin 2 in the vesicle membrane. The regulation and assembly of these proteins into the SNARE complex is essential to neuronal fusion, and a number of critical effector proteins that function to mediate this process have been identified [44, 102, 127]. In the case of syntaxin, Munc18-1 is believed to control the configuration of syntaxin and thereby regulate its ability to assemble into the SNARE complex $[44,105,106]$. Less is known about the state of the vesicle-associated SNARE protein, synaptobrevin 2, which is generally thought to be unstructured prior to the SNARE assembly process.

The extravesicular SNARE domain of synaptobrevin 2 (syb) encompasses residues $30-85$ and is anchored to the membrane of synaptic vesicles through a single transmembrane helix near its $C$ terminal end (residues 95-116). In the absence of the transmembrane domain, synaptobrevin is reported to be almost completely unstructured in solution. [53, 128]. However, a high-resolution nuclear magnetic resonance (NMR) study of full-length syb in dodecylphosphocholine (DPC) micelles [54] indicated that stretches of the SNARE motif are helical. This micelle structure is shown in Figure 27a. The N-terminal half of the SNARE motif 


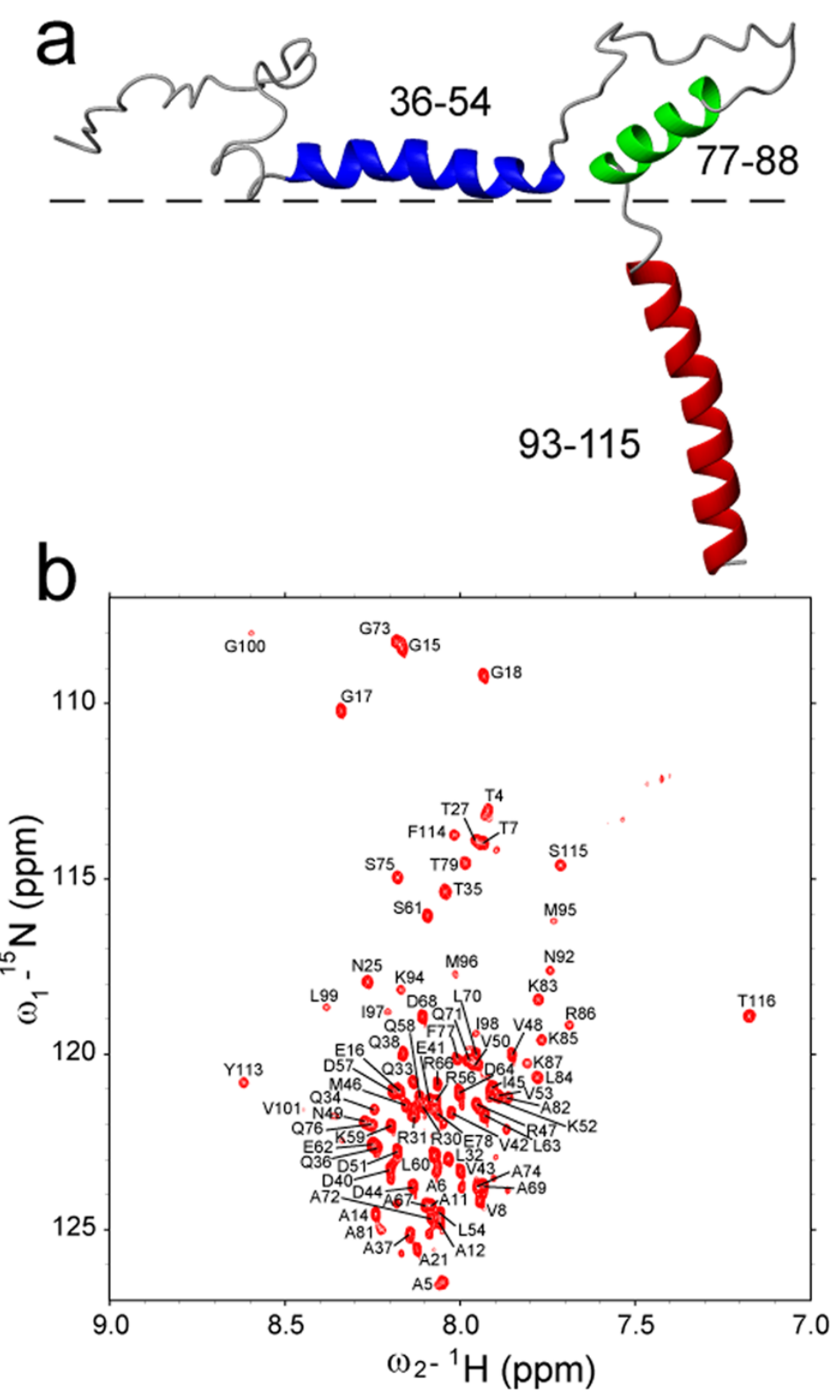

Figure 27. (a) Model obtained previously by solution NMR in DPC micelles for full-length synaptobrevin (Protein Data Bank entry 2KOG) [54]. Three segments of the protein assume a helical structure: the transmembrane segment (residues 93-115, red), the juxtamembrane coupling helix (residues $77-88$, green), and the $\mathrm{N}$-terminal half of the SNARE motif (residues $36-54$, blue). (b) ${ }^{15} \mathrm{~N}-{ }^{1} \mathrm{H}$ HSQC NMR spectrum of Syb(1-116) in DMPC/DHPC $(\mathrm{q}=0.33)$ bicelles at $40{ }^{\circ} \mathrm{C}(66$ mM DMPC and $200 \mathrm{mM}$ DHPC). 
(residues 36-54), the juxtamembrane coupling region (residues 77-88), and the transmembrane domain (residues 93-115) are $\alpha$-helical, while the remainder of the protein is unstructured. The SNARE motif is also observed to be partially helical for syb lacking the transmembrane anchor in the presence of DPC micelles; however, helical content is not observed for the soluble fragment in the absence of DPC micelles, con fi rming the earlier studies. The work in micelles indicates that syb has a substantial $\alpha$-helical structure in selected regions, which might play a role in protein-protein recognition and the nucleation of SNARE complex formation. In contrast, a more recent study of full-length syb in nanodiscs and in phospholipid bilayers indicates that residues 1 to $\sim 75$ are unstructured while the transmembrane domain of syb was not resolved [52]. This suggested that the helical content found in DPC may be induced by the micelle environment, producing helical structure in the SNARE motif and an interfacial association that may not occur in more native like environments.

In this work, we examine the structure and configuration of syb using both NMR and EPR spectroscopy in bilayer and bicelle environments and compare the result with that found previously by NMR in DPC micelles. In agreement with a more recent study, [52] we find that DPC micelles induce helical structure and interfacial association of the SNARE motif when compared to more native environments such as bilayers and bicelles. However, NMR spectroscopy demonstrates that in bicelles, syb is in conformational exchange and that some residual helical content is present. Moreover, EPR spectroscopy from 21 sites along syb provides strong evidence that even in a lipid bilayer, the SNARE motif of syb exchanges between aqueous and membrane environments. Although the SNARE 
motif is largely disordered, segments of the SNARE motif transiently associate with lipid bilayers, and this association is enhanced as one proceeds from bilayer to bicelle and micelle environments. Thus, the interfacial association and structure that are seen in DPC micelles persists in lipid bilayers, although to a greatly reduced extent. Our results suggest that the larger helical content and membrane association that occurs in micelles versus bilayers are a result of the sensitivity of amphipathic regions of the SNARE domain to interfacial defects and the curvature of the interface. The transient interfacial association and/or helix formation in the SNARE-forming motif of syb may modulate the availability of synaptobrevin and its ability to assemble into the SNARE core complex.

\subsection{EXPERIMENTAL PROCEDURES}

\section{Protein Expression and Bicelle Sample Preparation.}

Syb (1 -116) from Rattus norvegicus was expressed in BL21-(DE3) cells under the control of the T7 promoter (pET28a) and purified as described previously [35, 129]. The QuikChange polymerase chain reaction method (Agilent Technologies, Wilmington, DE) was used to introduce single-cysteine mutations into syb. Synaptobrevin mutants for EPR spectroscopy were expressed in lysogeny broth (LB) medium with $40 \mathrm{mg} / \mathrm{L}$ kanamycin. When the cells reached a cell density of 0.8 -1.0 , expression was induced with $0.4 \mathrm{mM}$ isopropyl thio- $\beta$ galactoside. The cells were incubated at $20^{\circ} \mathrm{C}$ overnight and then harvested by centrifugation at $3500 \mathrm{~g}$. For NMR measurements, isotope labeling was accomplished in EMBL medium in 100\% D 2 O supplemented with $\left({ }^{15} \mathrm{NH}_{4}\right)_{2} \mathrm{SO}_{4},{ }^{13} \mathrm{C}$-labeled glucose, and ${ }^{2} \mathrm{H}-{ }^{13} \mathrm{C}$-, and ${ }^{15}$ N-labeled $10 \%$ Bioexpress. For both EPR and NMR spectroscopy, syb was 
purified in the presence of $1 \%$ sodium cholate and exchanged into $0.1 \%$ dodecylphosphocholine (DPC) while bound to a Ni affinity column. The eluted protein from the Ni affinity column was concentrated and then digested at $4{ }^{\circ} \mathrm{C}$ overnight by thrombin to remove the six-His tag.

To make bicelle samples for NMR measurements, detergent exchange and buffer exchange were conducted via size-exclusion chromatography (Superdex200 10/300 column) with a pH 6.5 buffer containing 1\% dihexanoylphosphatidylcholine (DHPC), $20 \mathrm{mM}$ bis-tris, $50 \mathrm{mM} \mathrm{NaCl}, 5 \mathrm{mM}$ DTT, and $1 \mathrm{mM}$ EDTA. Only the major peak, but not the trailing shoulder, was collected and then concentrated to ensure the separation of DPC-bound syb from DHPC-bound syb. DHPC concentrations usually varied between 150 and $300 \mathrm{mM}$ in the final concentrated syb/DHPC sample. One-dimensional NMR was employed to accurately determine the DHPC concentrations in such samples by comparing the detergent acyl proton signals against corresponding resonances in a series of standard DHPC samples. On the basis of the measured DHPC concentrations, amounts of dimyristoylphosphatidylcholine (DMPC) corresponding to predetermined bicelle $\mathrm{q}$ factors were dispensed from a chloroform stock and dried under vacuum overnight. After the syb/DHPC sample had been mixed with the dried DMPC lipid film, homogeneous bicelle sample were achieved by multiple steps of freezing and thawing [130]. Multiple bicelle samples with q factors that vary from 0.25 to 0.5 were employed, and they were stable for a few months at a measuring temperature of $40^{\circ} \mathrm{C}$.

\section{NMR Spectroscopy.}

TROSY versions [131] of three-dimensional backbone experiments \{HNCA [132], 
NCACB [133], HNCO [132] and HN(CA)CO [134]\} with $\{2 \mathrm{H}, 13 \mathrm{C}, 15 \mathrm{~N}\}$ $\operatorname{Syb}(1-116)$ in $\mathrm{q}=0.33$ bicelles $(66 \mathrm{mM}$ DMPC and $200 \mathrm{mM}$ DHPC) were conducted on a Bruker Avance $800 \mathrm{MHz}$ spectrometer equipped with a cryoprobe. Secondary chemical shifts were evaluated as described previously [135]: $(\Delta \mathrm{C} \alpha-\Delta \mathrm{C} \beta)_{\mathrm{i}}$ was calculated as ${ }^{1 /}{ }_{3}\left(\Delta \mathrm{C}^{\alpha}{ }_{\mathrm{i}-1}+\Delta \mathrm{C}^{\alpha}{ }_{\mathrm{i}}+\Delta \mathrm{C}^{\alpha}{ }_{\mathrm{i}+1}-\Delta \mathrm{C}^{\beta}{ }_{\mathrm{i}-1}-\Delta \mathrm{C}^{\beta}{ }_{\mathrm{i}}-\Delta \mathrm{C}^{\beta_{\mathrm{i}+1}}\right)$. NMR dynamics measurements, including heteronuclear [130] ${ }^{15} \mathrm{~N} \mathrm{NOE},{ }^{15} \mathrm{~N} \mathrm{~T}_{1}$, and ${ }^{15} \mathrm{~N} \mathrm{~T}_{2}$ measurements [136], were also collected at $800 \mathrm{MHz}$. A $5 \mathrm{~s}$ saturation delay was used in the heteronuclear NOE experiment. Relaxation delay times of 10, 30, $70,150,300,600,1000$, and $1500 \mathrm{~ms}$ and $0,17,51,68,119,170,238$, and $494 \mathrm{~ms}$ were employed in the $T_{1}$ and $T_{2}$ experiments, respectively.

For CPMG relaxation dispersion experiments, bicelle samples with q values from 0.25 to 0.5 were employed at temperatures from 15 to $40{ }^{\circ} \mathrm{C}$ to determine the optimal conditions for such measurements. Although the relaxation behavior changes only slightly, the general trend is consistent throughout the surveyed conditions. Typically, the TROSY versions of CPMG relaxation dispersion experiments [137] were conducted on a Bruker Avance 600 or $800 \mathrm{MHz}$ spectrometer. $\mathrm{R}_{2, \text { eff }}$ was calculated on the basis of the equation [138]

$$
R_{2, \mathrm{eff}}=-\frac{1}{T_{\mathrm{CP}}} \ln \frac{I\left(\nu_{\mathrm{CPMG}}\right)}{I_{0}}
$$

where $\mathrm{I}\left(v_{\mathrm{CPMG}}\right)$ and $\mathrm{I}_{0}$ are peak intensities measured with and without the applied 40 ms constant time $\mathrm{CPMG}$ element, $\mathrm{T}_{\mathrm{CP}}$, respectively. Redundant measurements were performed to estimate standard deviations. Effective fields, $v_{\mathrm{CPMG}}$, as defined by $1 / 4$ $\tau_{\mathrm{CPMG}}$, ranged from 25 to $1000 \mathrm{~Hz}$, where $2 \tau_{\mathrm{CPMG}}$ was the time between the centers of two consecutive $180^{\circ}$ pulses. 
All spectra were processed and analyzed with NMRPipe [139] and Sparky [140]. Indirect dimensions in the three-dimensional experiments were processed with forward-backward linear prediction.

\section{Sample Preparation and EPR Measurements.}

The spin-labeled side chain R1 was attached to selected sites on syntaxin by adding $1 \mathrm{mg}$ of 1-oxyl-2,2,5,5-tetramethyl-3-pyrro-line-3-methyl methanethiosulfonate (MTSL) (Santa Cruz Biotechnology, Dallas, TX) in ethanol to the isolated cysteinecontaining protein and incubating the mixture overnight at $4{ }^{\circ} \mathrm{C}$. The unbound spinlabel was then removed using a HiPrep 26/10 desalting column (GE Healthcare, Piscataway, NJ) using a buffer that consisted of $20 \mathrm{mM}$ MOPS, $139 \mathrm{mM} \mathrm{KCl}, 12$ $\mathrm{mM} \mathrm{NaCl}$, and $0.1 \%(\mathrm{w} / \mathrm{v}) \mathrm{DPC}(\mathrm{pH} 7.3)$, and the eluted protein was concentrated by ultrafiltration. These syb samples in DPC were typically at protein concentrations of 50-200 $\mu \mathrm{M}$ and were used for EPR spectroscopy.

Bicelle samples containing syb for EPR were prepared by first reconstituting the protein into DMPC (Avanti Polar Lipids, Alabaster, AL). The lipid in chloroform was dried under vacuum to produce a film of DMPC, and this sample was rehydrated with $20 \mathrm{mM}$ MOPS, $139 \mathrm{mM} \mathrm{KCl}$, and $12 \mathrm{mM} \mathrm{NaCl}(\mathrm{pH}$ 7.3). A specific volume of syb in DPC was then added to the lipid suspension, and the solution was then incubated overnight at $4{ }^{\circ} \mathrm{C}$ and dialyzed twice in $20 \mathrm{mM}$ MOPS, $139 \mathrm{mM} \mathrm{KCl}$, and $12 \mathrm{mM} \mathrm{NaCl}(\mathrm{pH} 7.3)$ to remove the detergent. The resulting vesicle suspension was spun down at $500000 \mathrm{~g}$, and the lipid concentration was evaluated by a phosphate assay. The desired amount of DHPC was then added to achieve the appropriate detergent:lipid ratio, and the mixture was subjected to bath sonication for $20 \mathrm{~min}$. The final protein concentration for EPR 
was $20-60 \mu \mathrm{M}$.

To prepare synaptobrevin in lipid vesicles, a POPC/POPS (3:1) suspension was prepared by drying the lipids in chloroform (Avanti Polar Lipids) into a film followed by hydration in a buffer that consisted of $20 \mathrm{mM}$ MOPS, $139 \mathrm{mM} \mathrm{KCl}$, and $12 \mathrm{mM} \mathrm{NaCl}(\mathrm{pH} 7.3)$. Appropriate quantities of synaptobrevin in DPC were then added to the lipid mixture followed by incubation for $2 \mathrm{~h}$ at room temperature and dialysis against $20 \mathrm{mM}$ MOPS, $139 \mathrm{mM} \mathrm{KCl}$, and $12 \mathrm{mM} \mathrm{NaCl}(\mathrm{pH} \mathrm{7.3)}$. Following removal of the detergent, the vesicles were pelleted by centrifugation at $500000 \mathrm{~g}$ for $20 \mathrm{~min}$. The protein:lipid ratio was approximately 1:1000, and the protein concentration was in the range of $40-100 \mu \mathrm{M}$.

For the continuous wave measurements, samples were loaded into glass capillaries with inner and outer diameters of 0.6 and $0.84 \mathrm{~mm}$, respectively (VitroCom, Mountain Lakes, NJ). The measurements were performed on a Bruker EMX spectrometer with a room-temperature ER 4123D dielectric resonator using a $2 \mathrm{~mW}$ incident microwave power and a $1 \mathrm{G}$ modulation amplitude. Amplitudes for the EPR spectra were taken from spectra normalized by spin number. The power saturation experiments were performed on Bruker EMX as described elsewhere [18], except the NiEDDA concentration was kept at $10 \mathrm{mM}$. All EPR spectra and data were processed using LabView programs provided by C. Altenbach (University of California, Los Angeles, CA).

Simulations of EPR spectra to obtain motional rates for the nitroxide, motional populations, and hyperfine coupling constants were conducted using a LabView program, MultiComponent, provided by C. Altenbach. This program implements a program for fitting slow motional EPR spectra written by Freed and 
co-workers [79] The spectra were adequately fit by assuming the presence of two motional components having isotropic rates. The initial magnetic parameters used in the fits were taken from those obtained previously for R1 on aqueous exposed helical sites [126].

\subsection{RESULTS}

\section{Synaptobrevin Has a Different Structure in Bicelles and Micelles.}

To compare the structure of rat synaptobrevin 2 (syb) in micelle and bicelle environments, we obtained two-dimensional HN TROSY spectra of syb in small (q $=0.33$ ) bicelles (Figure 27b). This bicelle environment yielded spectra having a resolution and a signal-to-noise ratio similar to those previously obtained for syb in DPC micelles [54]. The spectra in bicelles were reassigned using ${ }^{2} \mathrm{H}-,{ }^{13} \mathrm{C}-$, and ${ }^{15} \mathrm{~N}-$ labeled samples and similar strategies as previously described for the DPC samples [54]. The assignments are shown in Figure $27 \mathrm{~b}$. The protein secondary structure was semiquantitatively assessed by examining the difference between secondary $\mathrm{C} \alpha$ and $\mathrm{C} \beta$ chemical shifts as described previously [135], and Figure 28 shows a comparison between the secondary $\mathrm{C} \alpha-\mathrm{C} \beta$ shift differences for syb in DPC and bicelles. While the chemical shifts in the TM and coupling helices were similar in micelles and bicelles, shift differences of $\geq 2 \mathrm{ppm}$ are observed for syb in DPC in the region of residues $37-53$, indicating the presence of an $\alpha$-helical structure; however, the same region in bicelles displays secondary $\mathrm{C} \alpha-\mathrm{C} \beta$ differences of $\leq 1$ ppm and is consistent with random coil structure. This finding is generally consistent with an NMR study in nanodiscs indicating that syb has a random coil structure in the SNARE motif, although signals in the coupling and TM helices 


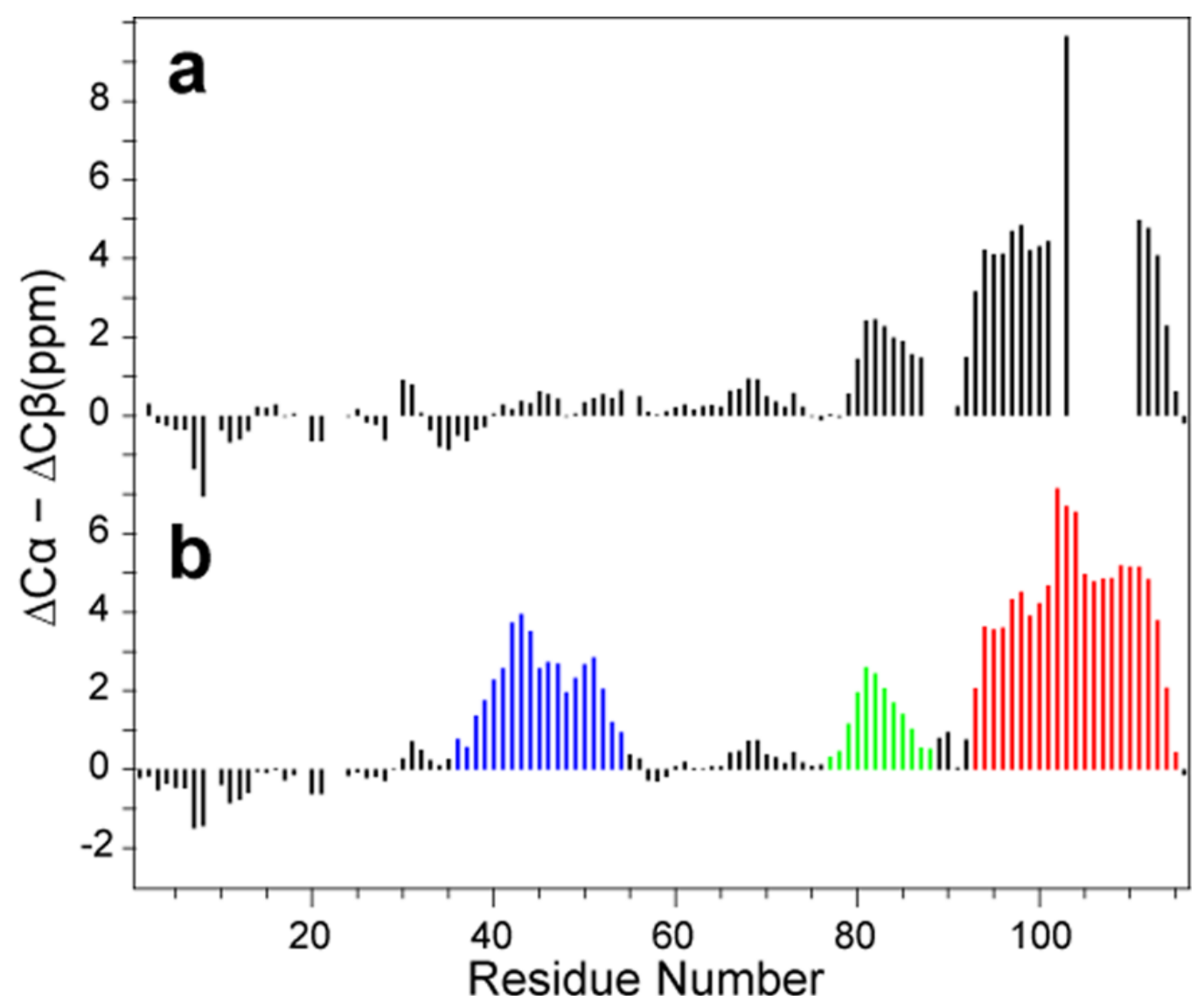

Figure 28. Chemical shift index obtained from $\mathrm{C} \alpha$ and $\mathrm{C} \beta$ resonances for syb in (a) DMPC/DHPC bicelles $(q=0.33)$ and $(b)$ DPC micelles. 
were not resolved in this study [52]. Several resonances in the C-terminal half of the transmembrane helix that are resolved in DPC are not resolved in bicelles.

More quantitative methods for determining secondary structure from chemical shifts have been devised [141, 142], and they yield results similar to those shown in Figure 28. Here, backbone $\mathrm{HN}, \mathrm{C} \alpha, \mathrm{CO}$, and $\mathrm{N}$ and side chain $\mathrm{C} \beta$ chemical shifts were used with the delta2D method [141] and are shown in Figure S6 of the Supporting Information. While the method suggests that syb in bicelles is largely random coil, the analysis indicates that a small $(<10 \%)$ amount of helix may be present at and near residue 45 in bicelles, and that there may be a small $(<20 \%)$, short stretch (three to five residues) of helix at and near residue 30 that is not seen in micelles or in solution. Thus, while the region encompassing residues $37-53$ shows a greater propensity for helical structure in DPC micelles than in low-q bicelles, the delta2D method suggests that a small helical population may be in equilibrium with a random coil structure in some regions of synaptobrevin.

${ }^{15}$ N Spin Relaxation Reveals Conformational Exchange in Regions of BicelleBound Synaptobrevin.

Nuclear spin relaxation rates are sensitive to local order and dynamics, and ${ }^{15} \mathrm{~N}$ spin-lattice $\left(R_{1}\right)$ and spin-spin $\left(R_{2}\right)$ relaxation rates were measured in bicelles $(q=$ 0.33 ) to explore the dynamics of syb. As shown in Figure $29 a$, elevated $R_{2} / R_{1}$ values are observed for residues 77-116, suggesting that there is a higher degree of local order and/or a shift in spectral density to lower frequency compared to those of residues 1-25. This is consistent with the known random coil structure of residues $1-25$ and the $\alpha$-helical structure of most residues in the region of residues 77-116, which are in the proximity of or inserted into the bicelle. Residues 37-54 

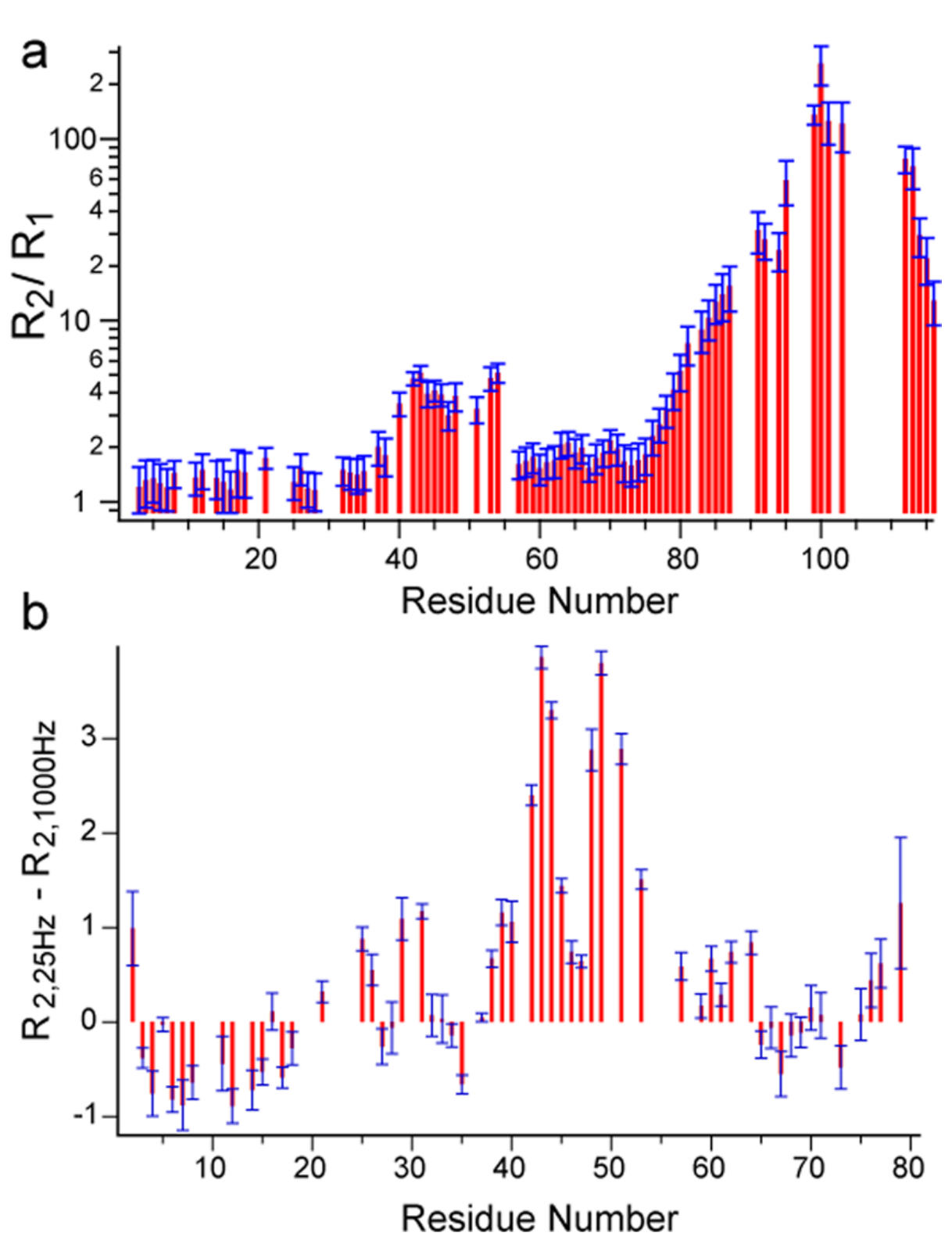

Figure 29. (a) Ratio of $15 \mathrm{~N} \mathrm{R} 2 / \mathrm{R} 1$ relaxation rates plotted for syb(1-116) in small DMPC/DHPC bicelles $(q=0.33)$ measured at $40{ }^{\circ} \mathrm{C}$ and $800 \mathrm{MHz}$. (b) Change in the spin-spin relaxation rates at two extreme CMPG pulse frequencies of 25 and $1000 \mathrm{~Hz}$, measured at $25^{\circ} \mathrm{C}$ and $800 \mathrm{MHz}$, for $\mathrm{syb}(1-116)$ in DMPC/DHPC bicelles $(q=0.5)$. 
also exhibit $R_{2} / R_{1}$ values that are elevated relative to those of residues $1-25$ but $R_{2} / R_{1}$ values smaller than those observed for the transmembrane $\alpha$-helix (residues 93-115). In contrast, the rest of SNARE motif residues 55-76 showed relatively small $R_{2} / R_{1}$ values, similar to that of residues $1-25$. These $R_{2} / R_{1}$ values are consistent with a model for the region of residues 37-54 in which a small population of syb assumes an $\alpha$-helical form and/or is interacting with the bicelle. To test for exchange, the dependence of the ${ }^{15} \mathrm{~N} \mathrm{R}_{2}$ on the refocusing pulse repetition rate (relaxation dispersion) for syb in bicelles was examined and is shown in Figure 29b. Additional relaxation dispersion data at lower temperatures are shown in Figure S7 of the Supporting Information. The observed dependence of the ${ }^{15} \mathrm{~N}$ spin-spin relaxation rate on CPMG pulse spacing for several residues between positions 42 and 52 indicates that these residues undergo conformational (random coil- $\alpha$-helical transitions) and/or environmental exchange (binding on and off the bicelle). The small difference in $\mathrm{R}_{2}$ values obtained for the different CPMG frequencies suggests that the exchange process is relatively fast (about $10^{5} \mathrm{~s}^{-1}$ ). Unfortunately, the small amplitude of the relaxation dispersion precluded a more detailed quantitative analysis of the dynamics.

\section{EPR Spectra of Synaptobrevin Are a Result of Two Motional Components}

\section{That Vary as a Function of the Curvature of the Environment.}

To examine the configuration and dynamics of synaptobrevin on lipid bilayers, the native cysteine at position 103 was mutated to alanine, and 21 sites in the SNARE motif were labeled with the spin-labeled side chain R1 (see Figure 30a). Shown in Figure $30 \mathrm{~b}$ are representative EPR spectra from synaptobrevin in which the R1 side chain is placed at the indicated position. These spectra, which have been 

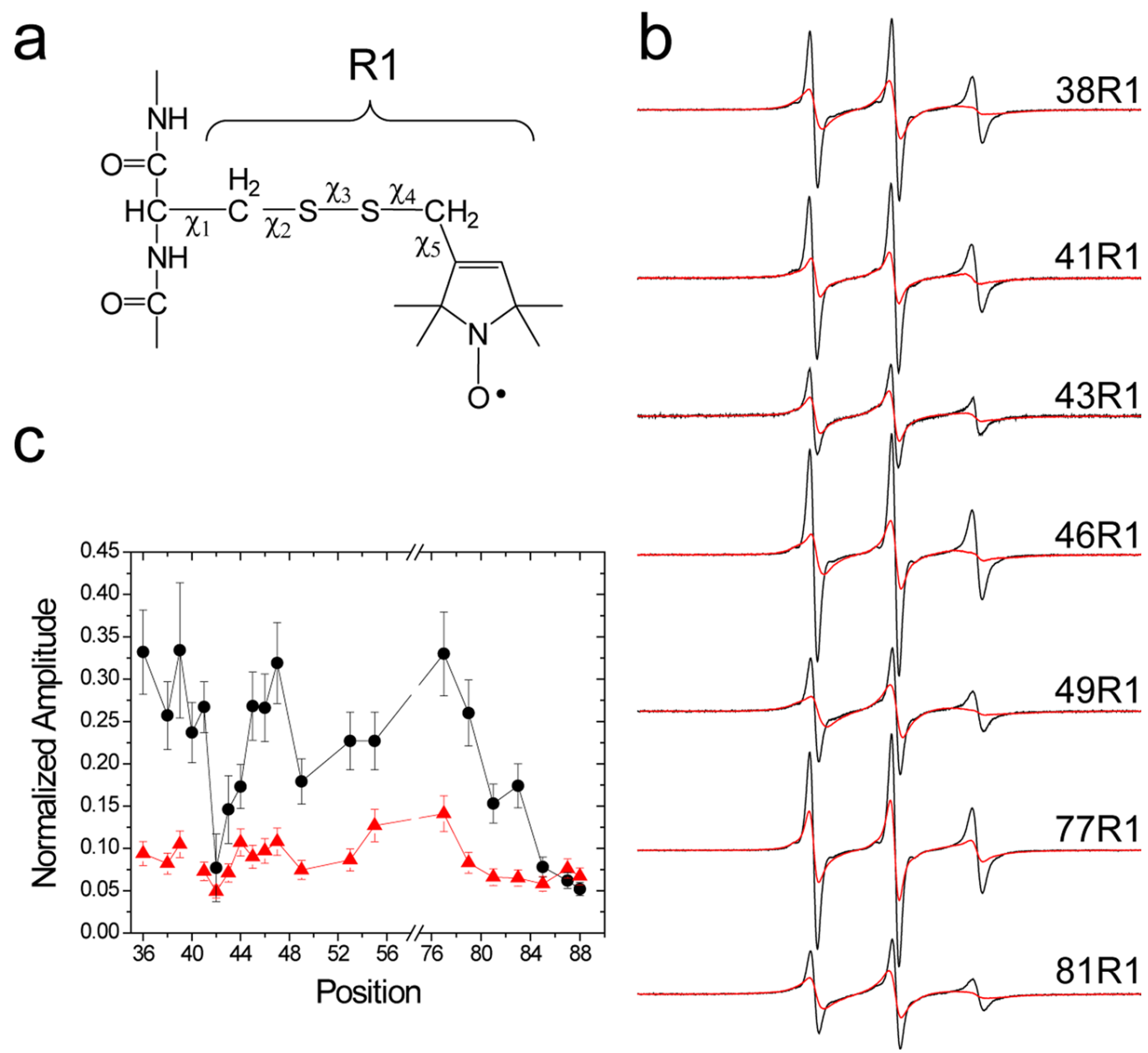

Figure 30. (a) Spin-labeled side chain R1 produced by the reaction of the MTSL reagent with a cysteine side chain. For this label, there are in principle five rotatable bonds linking the spin-label to the protein backbone; however, under most conditions, motion about $\mathrm{X} 1-\mathrm{X} 3$ is limited. (b) Selected normalized X-band EPR spectra from the spin-labeled sites within the SNARE motif of rat synaptobrevin 2 reconstituted into either POPC/ POPS (3:1) bilayers (black trace) or docecylphosphocholine micelles (red trace). Spectra are all $100 \mathrm{G}$ scans. (c) Normalized amplitudes of the EPR spectra as a function of position along synaptobrevin 2 . These amplitudes provide a relative measure of the motional averaging of the R1 side chain where the more motionally averaged side chains have the highest intensities. 
normalized by total spin number, are shown for the protein incorporated into POPC/POPS bilayers and DPC micelles. A complete set of EPR spectra are provided in Figure S8 of the Supporting Information. To provide a relative measure of the mobility of the R1 side chain along synaptobrevin, the peak-to-peak amplitudes are plotted in Figure 30c for the syb R1 mutants in bilayers and DPC micelles.

Two features are immediately obvious from the EPR spectra shown in Figure 30 (and Figure S8 of the Supporting Information). First, the normalized amplitudes in DPC are dramatically different from those in bilayers and indicate that that motion of the spin-labeled side chain is slowed in DPC. Second, there is considerable variation in the amplitudes of the synaptobrevin spectra as a function of position in the POPC/POPS sample. Sites $42-44$ as well as site 49 show intensities significantly lower than those at other positions on the SNARE motif. At a minimum, this indicates that the SNARE motif in syb is not uniformly unstructured in the presence of lipid bilayers. Sites $81-88$ also have lower intensities, and this is likely due to membrane insertion of the region adjacent to the TM domain [143].

The EPR spectra for the SNARE motif in bilayers appear to be highly mobile and indicative of an unstructured protein segment. This is at first glance consistent with the recent NMR results for synaptobrevin in nanodiscs [52] as well as an earlier EPR study on synaptobrevin in bilayers [143]. However, a closer examination of the EPR line shapes indicates that they are composed of at least two motional components. Shown in Figure 31a are EPR spectra for sites 49 and 85 and fits using an EPR simulation package based upon the MOMD approach developed 

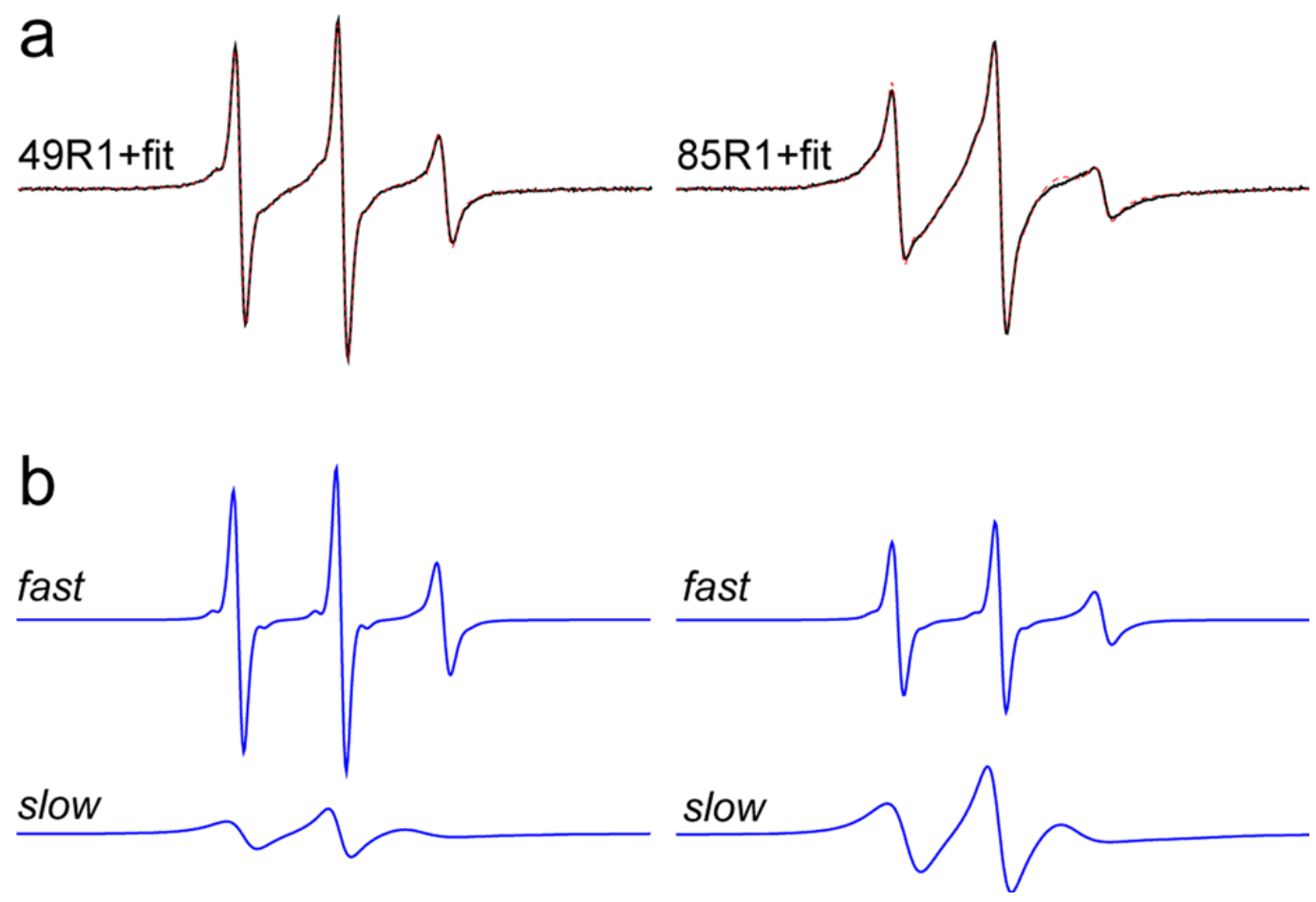

Figure 31. (a) EPR spectra for 49R1 and 85R1 in POPC/POPS bicelles (black trace) along with simulations (red dashed lines) that reproduce these spectra. (b) Spectra corresponding to the fast and slow components that when added produce the simulations shown in panel a for positions 49 and 85 . For 49R1, the fast and slow components have correlation times of 0.44 and $2.7 \mathrm{~ns}$, respectively, which are at relative spin populations of 45 and 55\%, respectively. For $85 \mathrm{R} 1$, the fast and slow components have correlation times of 0.65 and $3.1 \mathrm{~ns}$, respectively, which are at relative spin populations of 20 and $80 \%$, respectively. 
by Freed and co-workers (see Experimental Procedures). For these spectra, the simulations required two motional components to obtain a reasonable fit but were not improved by including an additional motional component. Shown in Figure 31b are the two components required for the fits shown in Figure 31a. The correlation times and fractions of the two components that reproduce the spectra are given in the legend. The fast motional component in these spectra is consistent with that expected for an unstructured segment that lies in the aqueous phase, and the slower motional component is similar to spectra obtained from peptides that are associated with the membrane-solution interface $[144,145]$. The variation in the normalized amplitude along the SNARE motif of synaptobrevin (Figure 30c) results in part from differences in these two motional populations, so that the sites having the largest fraction of the slower-moving component have the lowest amplitudes. The differences between bilayers and DPC also result from a greater population of the slow motional component in the micelle system (see below).

In addition to bilayer and micelle environments, spectra for several of these spin-labeled mutants were obtained in DHPC/ DMPC bicelles to provide a direct comparison with the NMR data shown above. The normalized EPR spectra in lipid bilayers and in $\mathrm{q}=2$ and $\mathrm{q}=0.4$ bicelles are shown in Figure S9 of the Supporting Information. In almost every case examined, the spectra in lipid have the highest normalized intensities, followed by the $\mathrm{q}=2$ and $\mathrm{q}=0.4$ bicelles. The EPR spectra obtained in bilayers, bicelles, and micelles were simulated, and the parameters for each component were averaged across multiple positions in the SNARE motif (legend of Figure 32). The resulting values of the $A_{z z}$ component of the hyperfine tensor, the correlation time, and the population of each component are shown in 
a
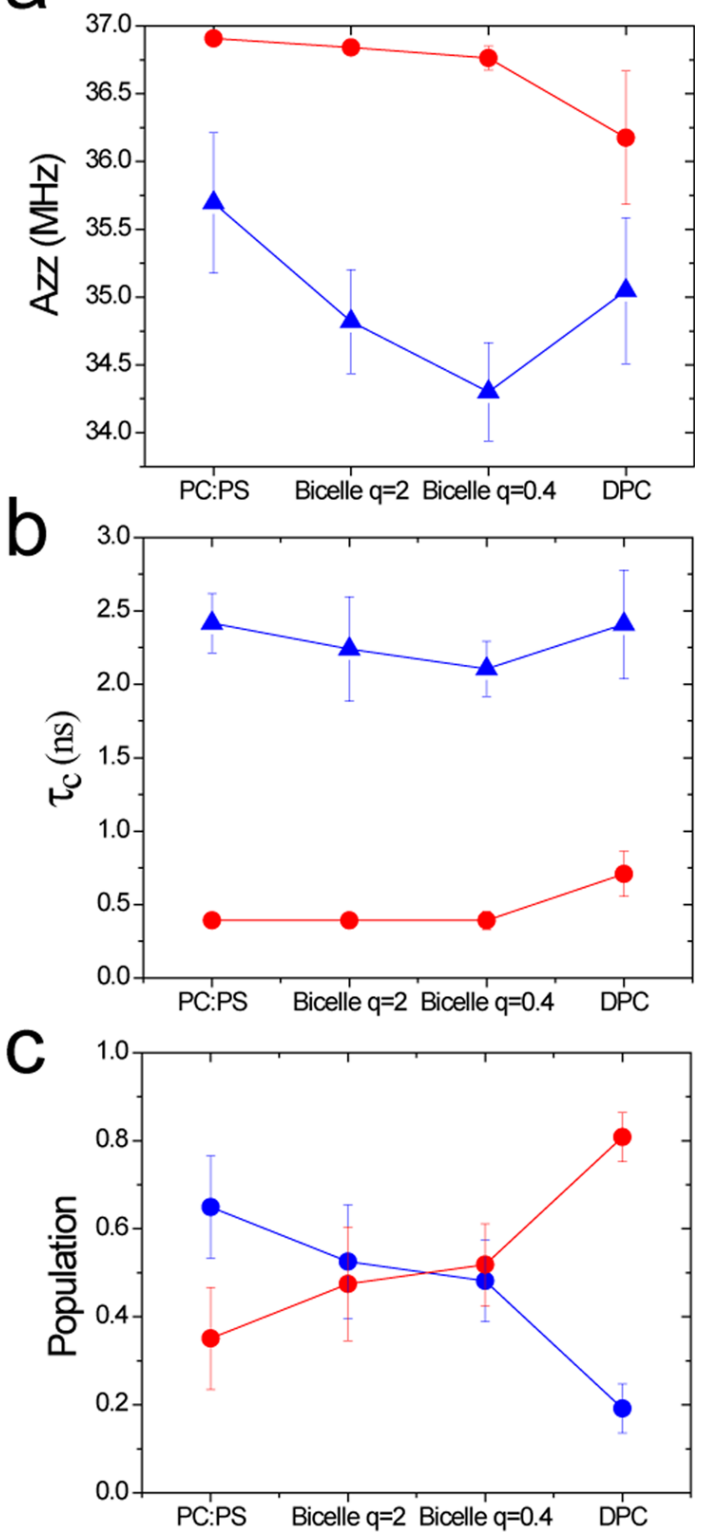

Figure 32. EPR parameters obtained from two-component fits such as those shown in Figure 31. (a) Values of the hyperfine coupling constant, $A_{z z}$, required to fit the fast (red) and slow (blue) motional components in the EPR spectra in PC/PS bilayers, $\mathrm{q}=2 \mathrm{DMPC} / \mathrm{DHPC}$ bicelles, $\mathrm{q}=0.33 \mathrm{DMPC} / \mathrm{DHPC}$ bicelles, and DPC micelles. (b) Values of the rotational correlation time, $\tau_{\mathrm{c}}$, required to fit the fast (red) and slow (blue) motional components in each environment. (c) Relative populations of the fast (red) and slow (blue) motional components required to fit 
the spectra in each environment. The error bars indicate the variation in values across all positions for which spectra were simulated. Parameters for the twocomponent fit included 15 positions in bilayers, 14 positions in $\mathrm{DPC}$, and 8 positions in bicelles. Results were not included for positions $42-44$ in bilayers, which appear to be more strongly associated with the interface. 
Figure 32. The average correlation times for the more mobile components were between 0.4 and $0.7 \mathrm{~ns}$, and average $\mathrm{A}_{\mathrm{zz}}$ values were between 36.2 and $36.9 \mathrm{G}$; these correlation times and $A_{z z}$ values, which are sensitive to polarity, indicate that the protein is unstructured and in an aqueous environment [146]. The values of $\mathrm{A}_{\mathrm{zz}}$ obtained for the slower component are consistent with an interfacial or hydrocarbon location for the R1 side chain; however, EPR line shapes and correlation times are more difficult to interpret for hydrocarbon-facing labels [147, 148] and the slower component might arise from an unstructured and/or helical backbone segment.

Remarkably, the correlation times of the two components do not significantly change when bilayer, bicelle, and micelle environments are examined (Figure 32b), but as indicated in Figure 32c, the populations of the two components change as a function of environment, so that the population of the slower- moving component is largest in DPC micelles and smallest in bilayers. These EPR spectra are consistent with exchange of the labeled syb on and off the interface at a rate that is slower than the EPR time scale $\left(<10^{8} \mathrm{~s}^{-1}\right)$. It should be noted that in addition to measurements in bilayers formed by POPC and POPS, several sites (36, 43, and 53) were also examined in bilayers formed from POPC, which yields a neutral bilayer interface. These spectra, which are shown in Figure S10 of the Supporting Information, were not altered by the absence of POPS, indicating that the membrane surface potential or surface charge density does not play a strong role in controlling the partitioning of the SNARE segment of syb.

The syb EPR spectra were power-saturated in the presence of $\mathrm{O}_{2}$ or Ni(II)EDDA to assess the local environment of the spin- labeled Syb in bilayers [95]. Spin-label depth parameter $\varphi$ is shown in Figure S11 of the Supporting 
Information as a function of label position. The depth parameter measurement will be dominated by the shorter correlation time component of the spectra because of the large amplitude and small line width of this component. Depth parameters for positions between 36 and 83 indicate a high degree of aqueous exposure with little bilayer contact, and this is consistent with an aqueous localization for the conformer giving rise to the fast component. The N-terminus of the syb transmembrane helix is at or near residue 92 , and the increase in the depth parameter that appears to begin near residue 85 or 87 is consistent with the interfacial or hydrocarbon localization for this end of the SNARE segment that was seen previously by using EPR spectroscopy [143].

These data demonstrate that differences in EPR spectra along the length of the SNARE motif as well as the differences among bilayer, bicelle, and micelle environments are a result of a shift in the populations of fast and slow motional components that are associated with aqueous and interfacial localization, respectively. The result indicates that the SNARE motif of synaptobrevin partitions between aqueous and interfacial locations, and that this partitioning is a function of the membrane or membrane mimetic system and the interfacial environment. The increase in the interfacial population as one proceeds from bilayer to bicelle to micelle phases suggests that the tendency of the SNARE motif to transiently associate is correlated with the curvature of the interface.

\subsection{DISCUSSION}

Previous work on full-length synaptobrevin indicated that the SNARE motif is unstructured in bilayers or bilayer-like environments, and it has been suggested that 
the interfacial association and helical content of this segment that are observed in micelles are artifacts of the micelle environment [52]. The work presented here reaches a somewhat different conclusion with regard to the state of the protein. The combination of NMR and EPR data indicates that membrane association and partial helical content for the SNARE motif of syb occur in every environment examined (bilayers to micelles) but do so to a greatly reduced extent as the interface becomes more planar. This dependence upon the curvature of the interface explains the differences observed by NMR among DPC [54], bicelles, and nanodiscs [52].

EPR spectroscopy is a good method for distinguishing conformational exchange events and populations of conformers [108, 149], and a careful examination of the EPR spectra from the SNARE-forming motif of syb reveals the presence of two motional components in every environment examined. These two components appear to arise from aqueous and membrane-associated protein, and the differences in the EPR spectra of syb in different interfacial environments are largely due to the populations of fast and slow motional components (Figure 32c). The slow component dominates the spectra from DPC micelles; the fast motion component dominates in phospholipid bilayers, and the two components make comparable contributions in bicelles. Similarly, the NMR data presented here in bicelles indicate that several residues, including residue 44 and nearby neighboring residues, are in exchange (Figure 29) and at least partially helical (Figure S6 of the Supporting Information), suggesting that the interfacial protein assumes a partial helical state. The highly resolved resonances in NMR and the two-component EPR spectra indicate that the rate at which the SNARE motif exchanges between aqueous and interfacial phases is slow on the EPR time scale but fast on the NMR 

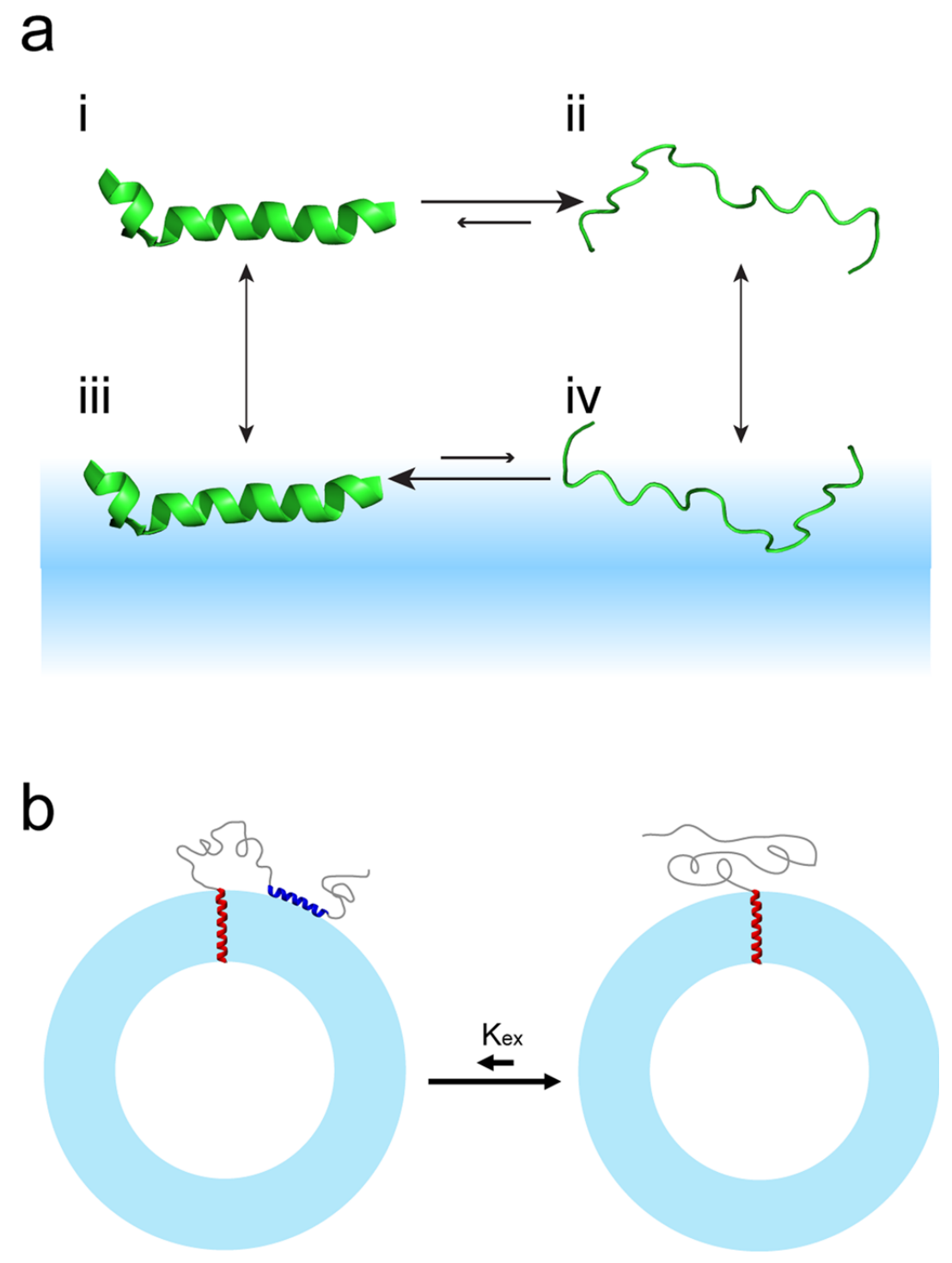

Figure 33. (a) Model based upon previous work [160] for the interfacial association of a helical segment that is in conformational exchange between folded (helical) and unfolded forms. In solution, the disordered form (ii) is favored relative to the helical form (i), while in the membrane, the equilibrium favors the helical form (iii) over the unfolded form (iv). (b) In the membrane, the equilibrium favors the unstructured aqueous form of the syb SNARE-forming segment; however, the SNARE-forming domain of syb samples the membrane and converts between helical and random forms, increasingly favoring the helical form in highly curved regions. 
time scale. This places the exchange rate in the range of $10^{5}-10^{8} \mathrm{~s}^{-1}$.

A model illustrating the equilibrium that would explain these data is shown in Figure 33. The slow components in the EPR spectra are best explained by the interfacial states (iii and iv in Figure 33a), and the fast component is due to the aqueous syb states ( $\mathrm{i}$ and ii in Figure 33a). When syb is in solution, the unstructured form of syb is highly favored, as indicated by the fact that little helical content is detected for the SNARE motif of syb in the absence of an interface. The fraction of $\alpha$-helical content found in the syb SNARE domain in DPC micelles and DHPC/DMPC bicelles by NMR (Figure 28b) roughly correlates with partitioning of the SNARE domain into the membrane mimetics observed by EPR, indicating that when the SNARE motif is associated with the interface, the helical form is strongly favored. However, this correlation is not always perfect. For example, when position 45 is examined in small DMPC/DHPC bicelles, the slow EPR component represents $\sim 50 \%$ of the total spins, while the population of helix determined by NMR is $<10 \%$. This difference might be a result of the spin-label or an indication that much of the bound component is not $\alpha$-helical and that unstructured and $\alpha$-helical forms coexist in the bound state.

An interesting observation revealed in the EPR spectra is that the fraction of protein associated with the interface increases as one moves from a bilayer to a bicelle to a micelle system (Figure 32c). Clearly, there are differences in the composition of these interfaces, including the lack of a glycerol backbone in the DPC micelle and the presence of unsaturated lipids in the bilayer phase. Conceivably, these differences might contribute to some of the differences that are observed. However, the differences in partitioning are correlated with the expected 
curvature of the interface in these systems, and this would appear to provide an explanation for the differences in helical content and partitioning. The SNARE domain has significant amphipathic character when it is $\alpha$-helical [54],and lipid interfaces will stabilize the helical form of these amphipathic sequences [150-153]. In addition, the interfacial insertion of an amphipathic $\alpha$-helix will be favored by the presence of defects (exposed hydrophobic area) at the membrane mimetic interface $[154,155]$. The relative level of interfacial surface area containing a defect should decrease in the following order: DPC micelles $>$ DHPC/DMPC bicelles $>$ PC/PS bilayers. The same order is observed for partitioning of syb between aqueous and membrane mimetic phases (Figure 32c).

EPR data for selected sites in synaptobrevin were presented previously and are generally in agreement with the data obtained here [143]. The EPR line shapes are dominated by narrow lines indicative of an unstructured protein segment, and power saturation indicates that the segment adjacent to the transmembrane helical domain is buried within the bilayer. However, there are some important differences in the conclusions reached. As indicated here, a close examination of EPR line shapes and their variability with environment indicates that the SNARE motif of synaptobrevin is in equilibrium between the aqueous phase and the lipid interface. In addition, depth parameters near the transmembrane segment measured (Figure S11 of the Supporting Information) do not suggest the helical pattern observed previously; however, the error in these measurements is relatively large, and sites placed in this region were limited and were not the focus of this study.

Membrane fusion is triggered by interactions of syb with plasma membrane SNAREs to form a SNARE complex. This interaction will be facilitated by 
collisions with the acceptor complex and may be nucleated by helix formation as suggested previously [156]; as a result, the kinetics of SNARE complex formation is likely to depend on the equilibria shown in Figure 33a. It is easy to imagine how the membrane partitioning of the SNARE motif and helix-coil transitions within this region might modulate fusion. Aqueous states I and ii would be more likely to collide with aqueous acceptor SNAREs, but the membrane-associated syb (states iii and iv) could function as sites of nucleation for SNARE complex assembly, both because they tend to be more helical and because t-SNAREs such as syntaxin may also associate with interfaces [157]. As shown here, the curvature of the interface and the presence of membrane defects will alter the aqueous-membrane partitioning of syb and thereby modulate the availability of syb for SNARE complex formation. As a result, the lipid composition at the focal site of fusion and the presence of curvature or curvature strain are expected to influence the kinetics of SNARE complex formation. The calcium sensor synaptotagmin 1 has been reported to modulate membrane curvature $[158,159]$, and the observations made here indicate how synaptotagmin 1 might play a role in modulating the SNARE assembly indirectly by changing the properties of the bilayer.

In summary, we have used a combination of NMR and EPR spectroscopy to examine the partitioning and structure of the syb SNARE motif in different membrane mimetic environments. The syb SNARE domain associates with the lipid interface when bound to DPC micelles but favors the aqueous phase in the presence of PC/PS bilayers. When reconstituted into DHPC/DMPC bicelles, the syb SNARE domain partitions roughly equally between the solution and the interface. Even in lipid bilayers, a substantial fraction of the syb SNARE motif is associated 
with the interface, indicating that syb is not exclusively unstructured in the aqueous phase as an isolated SNARE protein. The tendency to associate with the membrane interface is correlated with the level of exposed hydrophobic surface area or defects that are expected in this environment. As a result, the state of syb is likely to be influenced by the specific lipid composition and curvature at the focal site of fusion.

\subsection{ACKNOWLEDGMENTS}

We thank Christian Altenbach (University of California, Los Angeles, CA) for providing LabView software for EPR data analysis and simulations. All the NMR experiments, including sample preparation and experimental analysis, was performed by Binyong Liang and Jeffrey Ellena.

\subsection{ABBREVIATIONS}

CPMG, Carr-Purcell-Meiboom-Gill; DHPC, dihexanoyl-phosphatidylcholine; DMPC, dimyristoylphosphatidylcholine; DPC, dodecylphosphocholine; EPR, electron paramagnetic resonance; MOMD, microscopic ordering with macroscopic disordering; MOPS, 3-(N-morpholino)propanesulfonic acid; MTSL, methanethiosulfonate spin-label; NiEDDA, nickel(II)-ethylenediamine-N,N ， -diacetic acid complex; POPC, palmitoy-loleoylphosphatidylcholine; POPS, palmitoyloleoylphosphati-dylserine; R1, spin-labeled side chain produced by derivatiza-tion of a cysteine with MTSL; SDSL, site-directed spin labeling; SNAREs, soluble N-ethylmaleimide-sensitive factor attachment receptor proteins; syb, synaptobrevin 2; TROSY, transverse relaxation-optimized spectroscopy. 


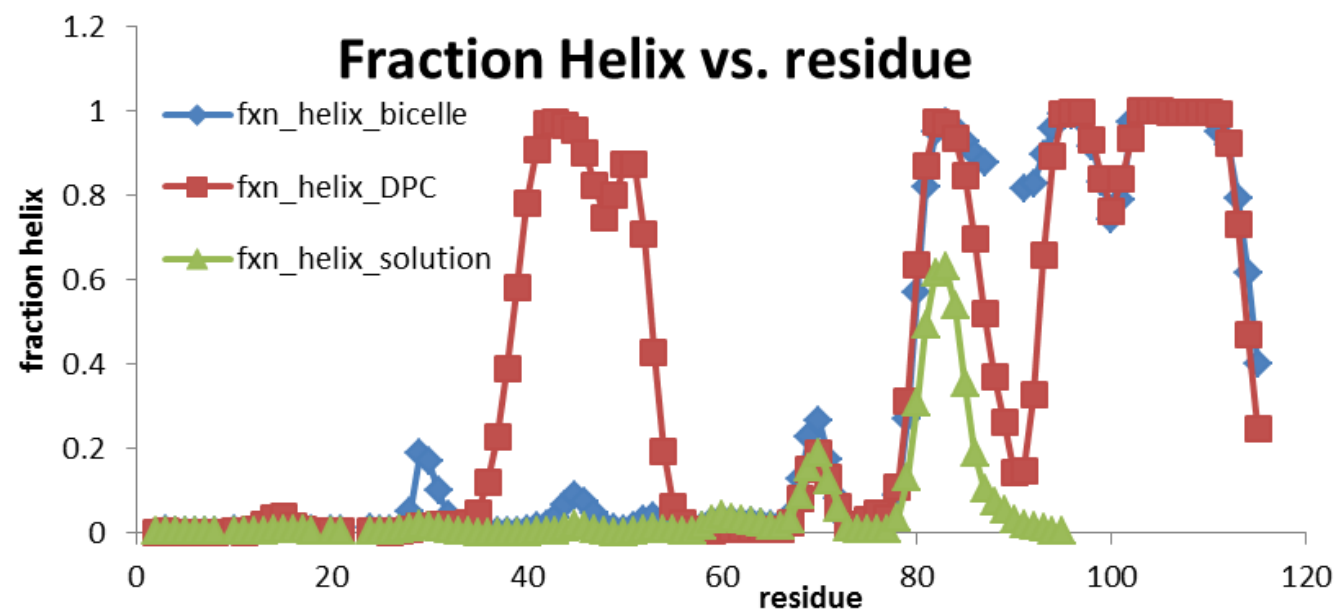

Figure S6. Backbone $\mathrm{HN}, \mathrm{C} \alpha, \mathrm{CO}$, and $\mathrm{N}$, and side chain $\mathrm{C} \beta$ chemical shifts plotted using the delta2D method (Camilloni et al., 2012) for synaptobrevin (1-116) in a DPC environment (red points), $\mathrm{q}=0.33$ bicelles (blue points) and for the soluble fragment of synaptobrevin (1-96) (green points). 

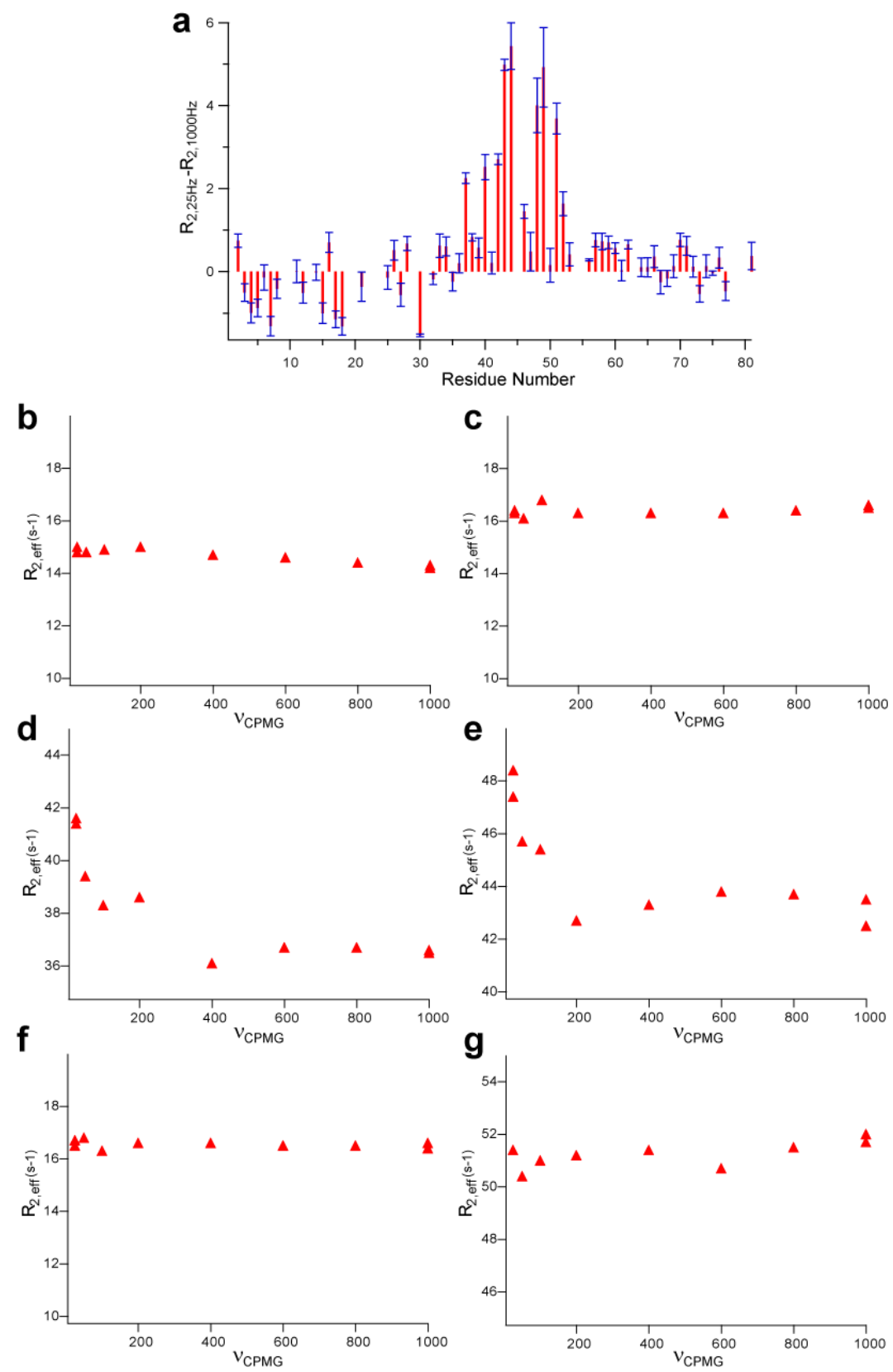

Figure S7. The CPMG experiment in bicelles was performed under a range of conditions. Here, the ${ }^{15} \mathrm{~N}-\mathrm{CPMG}$ experiment was performed at $15^{\circ} \mathrm{C}$ for syb $(1-116)$ in bicelles of DMPC/DHPC, $\mathrm{q}=0.5$, recorded at $800 \mathrm{MHz}$. In (a) is shown the change in the spin-spin relaxation rates at two extreme CMPG pulse frequencies of $25 \mathrm{~Hz}$ and 1000Hz. CPMG relaxation dispersion curves for selected residues S28 (b), L32 (c), V43 (d), N49 (e), D64 (f), and F77 (g). The CPMG effect is enhanced at lower temperature, indicating that the more ordered state is favored at lower temperature. The 
spin-spin relaxation rates (R2) are larger for d) and e) than for b), c) or f), which is expected since the later residues do not spend as much time associated with the membrane interface. 

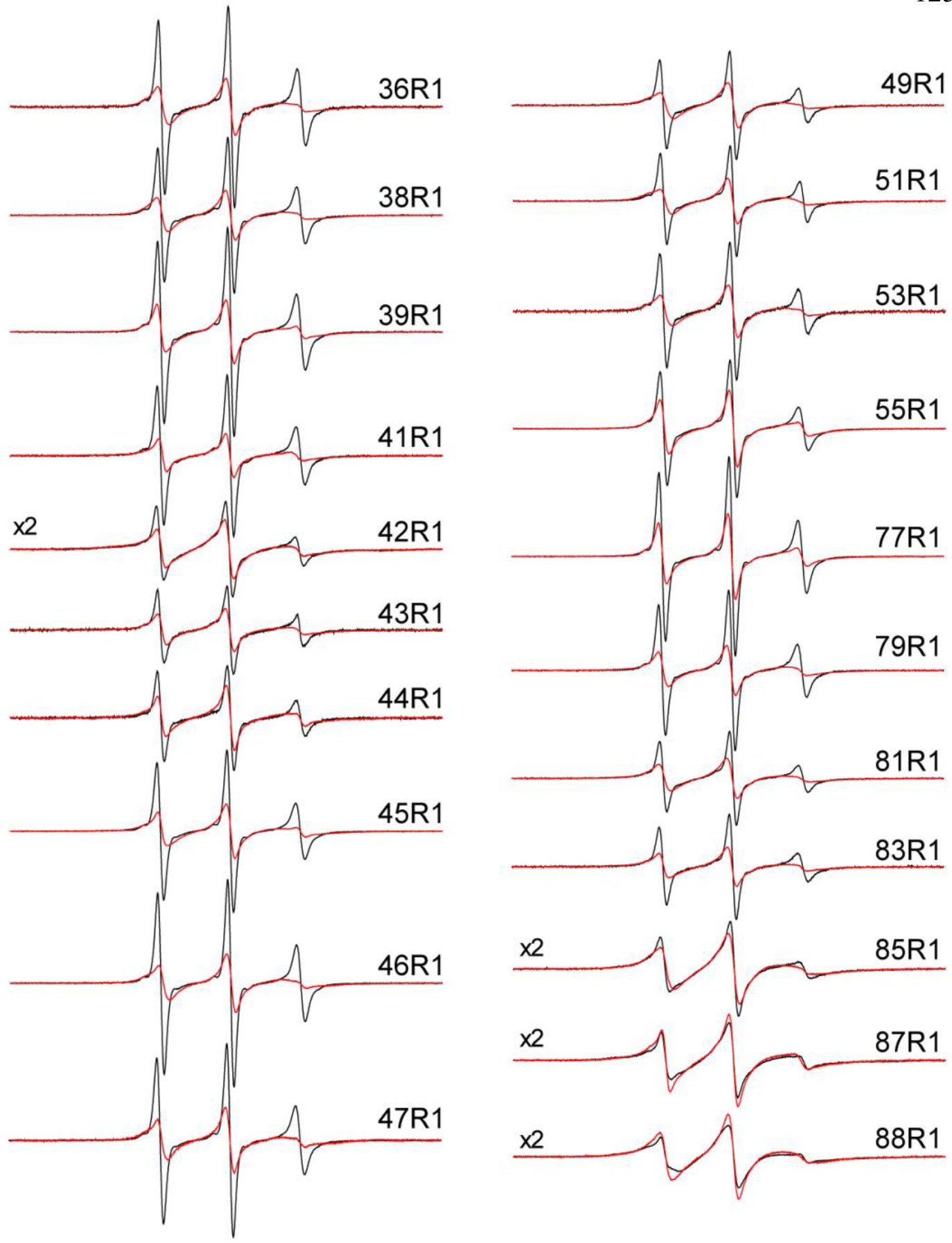

Figure S8. X-band EPR spectra for 21 positions along synaptobrevin when the spin labeled protein is reconstituted into either POPC:POPS (3:1) bilayers (black trace) or DPC micelles (red trace). Each spectrum is on the same vertical scale, except for 42R1, 85R1, 87R1 and 88R1, where the scale has been expanded by 2 fold. Each spectrum represents a 100 Gauss scan. 


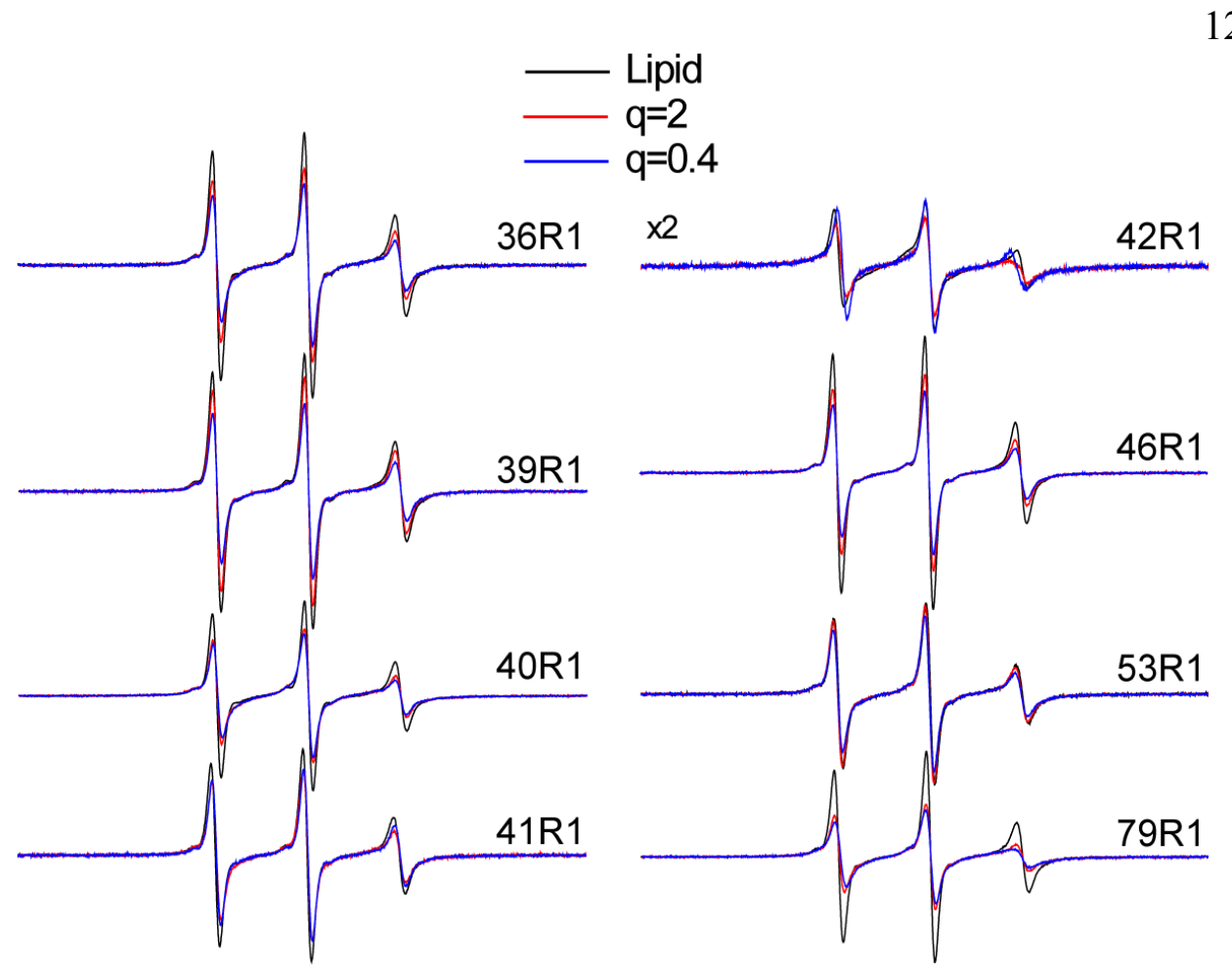

Figure S9. X-band EPR spectra obtained from several spin labeled sites within synaptobrevin 2 when the protein is reconstituted into POPC:POPS (3:1) bilayers (black trace), DHPC:DMPC $\mathrm{q}=2$ bicelles (red trace) and DHPC:DMPC $\mathrm{q}=0.4$ bicelles (red trace). The spectra are normalized for total spin concentration and each spectrum represents a scan of 100 Gauss. 


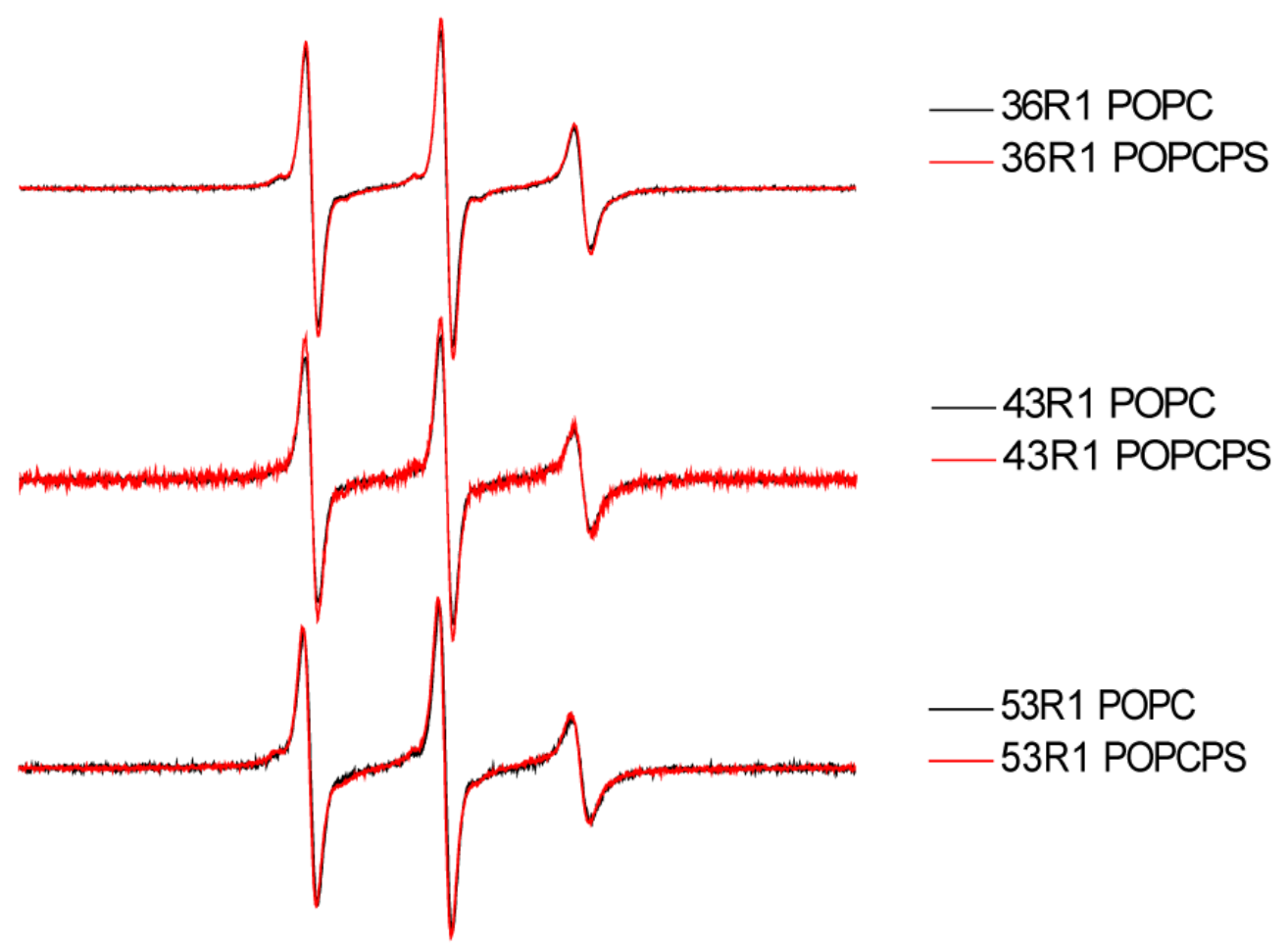

Figure S10. X-band EPR spectra for three sites in the SNARE region of membrane reconstituted synaptobrevin comparing the spectra in POPC bilayers (black traces) with that in POPC:POPS (3:1) bilayers (red traces). 


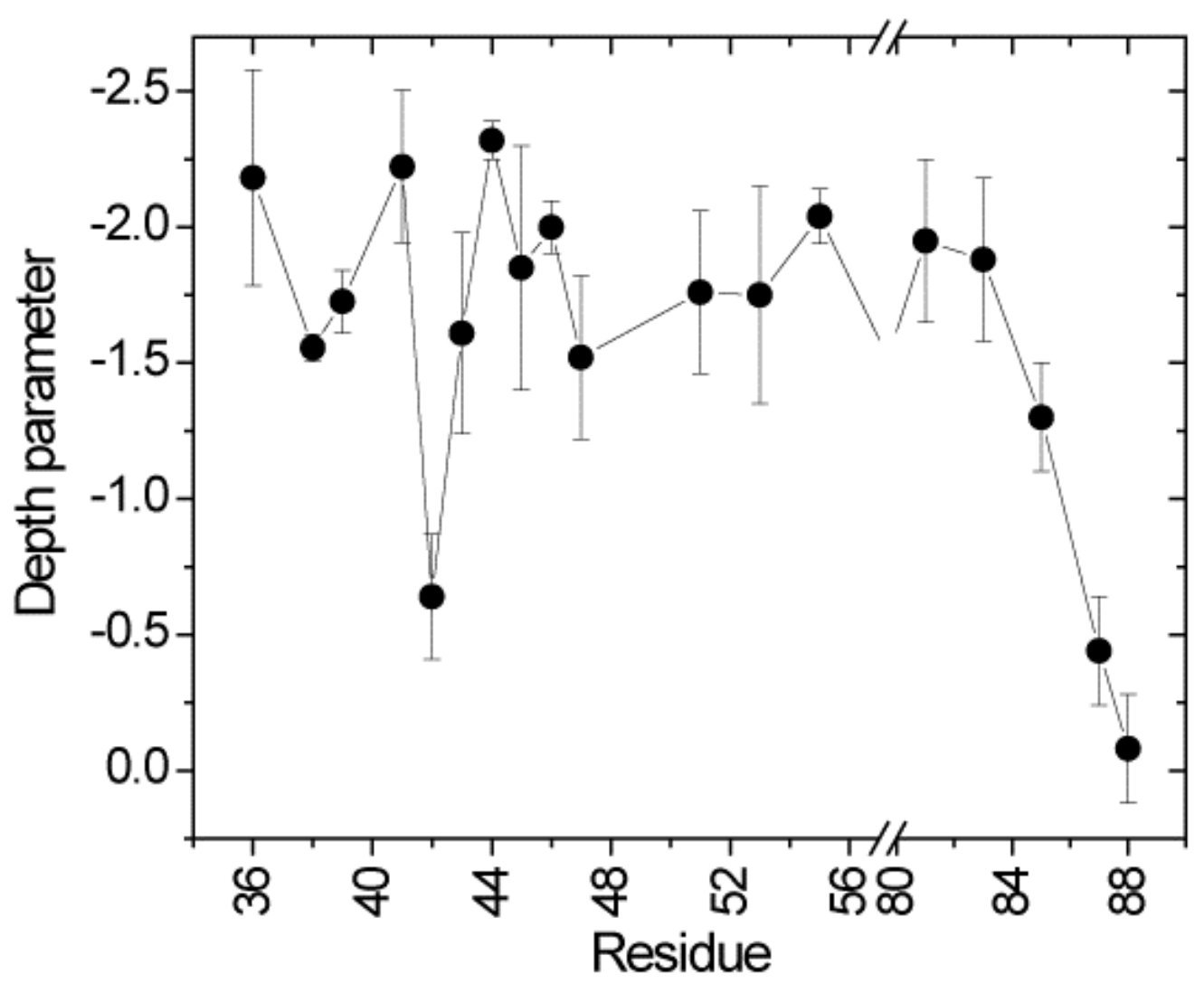

Figure S11. Membrane depth parameters obtained from progressive power saturation of the EPR spectra from synaptobrevin. Values near -2 indicate aqueous exposure, whereas values more positive than -2 indicate interfacial or hydrocarbon exposure of the spin labeled side chain. 
Chapter 5: Significance and Future Directions 
Syntaxin is arguably one of the more important elements of the neuronal fusion mechanism. It is the only neuronal SNARE that contains a separate domain in addition to its SNARE motif. This makes it a likely candidate for being regulated in neuronal fusion. Indeed, other crucial proteins of SNARE fusion: Munc13, Munc18 and synaptotagmin have been reported to interact with syntaxin. Despite two decades of intense research however, the exact mechanism of syntaxin function in neuronal fusion remains a mystery.

The EPR study presented here provided several insights that added to the general understanding of SNAREs in current literature. First of all, it presented the SNARE domain of syntaxin as being in a dynamic equilibrium that can be affected by Munc18. This effect of Munc18 extents to the start of the SNARE motif where SNARE complex formation is likely initiated. In addition, the pulse EPR data revealed the presence of conformational heterogeneity not revealed by the existing crystal structure of syntaxin and allowed as to visualize what its open state may look like. More elaborate DEER analysis also revealed how syntaxin's open-closed equilibrium is altered when the soluble syntaxin (which has been the subject of most previous investigations) is anchored to membrane, as it is in vivo.. The fact that syntaxin exhibits a much more open state on the bilayer will influence its interaction with its SNARE partners and regulatory proteins. This study also highlights the importance of studying a protein subject in an in vitro setup that matches its native environment as closely as possible. It is already clear that the bilayer will impact the syntaxin structure, and it is likely, that there are other factors that might have been omitted. Phenomena such as molecular crowding or posttranslational modifications that occur in the human cells can significantly change 
the property of a tested protein, but are usually overlooked in a typical in vitro protein study. It is inherently challenging to replicate the exact conditions in the cell due to the complexity of cytosol composition, which in fact exists in a dynamic rather then static equilibrium. One way to address this issue is to study the cells themselves; the emerging field of whole-cell biophysical methods may ultimately allow to overcome the problem of insufficient complexity of in vitro systems.

Another important finding pertains to the effect of different environments on the structure of synaptobrevin. The fact that a detergent will directly impact the protein that is being studied raises the problem of the non-native experimental conditions that was mentioned in the previous paragraph. Nevertheless, the EPR study of synaptobrevin on the lipid vesicle did reveal that this protein is largely unstructured, and that it can transiently contacts the lipid interface which has an impact on its structure, and in turn, could possibly change its affinity to other SNARE proteins.

A necessary next step to this EPR study will be to add additional elements of complexity to syntaxin and Munc18. The most logical one is SNAP25, the second SNARE that coexists with syntaxin on the presynaptic membrane. There is strong evidence in the literature that SNAP25 engages in interactions with syntaxin, likely before the onset of the calcium trigger. A future EPR study of these two SNAREs, possibly with Munc18, would yield valuable insight on what the “acceptor complex" of neuronal fusion may look like. Other element of complexity is the bilayer itself. While this study did highlight that the membrane-anchored syntaxin exhibits an open state, it will require additional DEER experiments to fully categorize this open state, and determine how it may be affected by Munc18. 
Future studies on the mechanism of neuronal fusion will expand on the measurements made here, as well as on other published works on neuronal fusion, and may with the aid of whole-cell approaches, result in full deciphering of this highly complex biological process. 


\section{References}

1. Katz B. The Release of Neural Transmitter Substances. Liverpool Univ. Press. (1969).

2. Kozlovsky Y., Chernomordik L., Kozlov M., Lipid Intermediates in Membrane Fusion: Formation, Structure, and Decay of Hemifusion Diaphragm. Biophys J. 83, 2634-2651 (2002).

3. Song L. Y., Ahkong Q. F, Georgescauld D., Lucy J. A. Membrane fusion without cytoplasmic fusion (hemi-fusion) in erythrocytes that are subjected to electrical breakdown. Biochim. Biophys. Acta. 62. 54 - 62 (1991).

4. Haucke V., Neher E. Sigrist S. J. Protein scaffolds in the coupling of synaptic exocytosis and endocytosis. Nature. 12, 127-38 (2011).

5. Hagler D., Goda Y. Properties of synchronous and asynchronous release during pulse train depression in cultured hippocampal neurons. $J$. Neurophysiol. 85, 2324-2334 (2001).

6. Sabatini B., Regehr W. G. 1996. Timing of neurotransmission at fast synapses in the mammalian brain. Nature. 384, 170-172 (1996).

7. Bollmann J. H., Sakmann B, Borst J. G. 2000. Calcium sensitivity of glutamate release in a calyx-type terminal. Science. 289, 953-957(2000).

8. Schneggenburger R., Neher E. Intracellular calcium dependence of transmitter release rates at a fast central synapse. Nature. 406, 889-893 (2000).

9. Chernomordik L. V., Kozlov M. M. Protein-lipid interplay in fusion and fission of biological membranes. Annu. Rev. Biochem. 72, 175-207 (2003).

10. Cohen F. S., Melikyan G. B. The energetics of membrane fusion from binding, through hemifusion, pore formation, and pore enlargement. $J$. Membr. Biol. 199, 1-14 (2004).

11. Wessels L., Elting M. W.,Scimeca D., Weninger K. Rapid Membrane Fusion of Individual Virus Particles with Supported Lipid Bilayers. Biophys J. 93, 526-538 (2007).

12. Sudhof T. C. The synaptic vesicle cycle: a cascade of protein-protein interactions. Nature. 375, 645-653 (1995).

13. Chen Y.A., Scheller R.H. SNARE-mediated membrane fusion. Nat. Rev. Mol. Cell Biol. 2, 98-106 (2001).

14. Rao S. K., Huynh C., Proux-Gillardeaux V., Galli T., Andrews N. W. Identification of SNAREs Involved in Synaptotagmin VII-regulated Lysosomal Exocytosis. J Biol Chem. 279, 20471-9 (2004).

15. Söllner T., Whiteheart S. W., Brunner M., Erdjument-Bromage H., Geromanos S., Tempst P., Rothman J. E. SNAP receptors implicated in vesicle targeting and fusion. Nature. 362, 318-324 (1993).

16. Augustine G. J. How does calcium trigger neurotransmitter release? Curr. Opin. Neurobiol. 11, 320-326 (2001).

17. Südhof, T. C. Neurotransmitter release. Handb. Exp. Pharmacol. 184, 1-21, (2008).

18. Frazier A. A., Roller C. R., Havelka J. J., Hinderliter A., Cafiso D. S. Membrane-bound orientation and position of the synaptotagmin I C2A domain by site-directed spin labeling. Biochemistry. 42,96-105 (2003). 
19. Bai J., Wang C. T., Richards D.A., Jackson M. B., Chapman E. R. Fusion pore dynamics are regulated by synaptotagmin t-SNARE interactions. Neuron. 41, 929-942 (2004).

20. Lai A. L., Huang H., Herrick D. Z., Epp N., Cafiso D.S. Synaptotagmin 1 and SNAREs form a complex that is structurally heterogeneous. J. Mol. Biol. 405, 696-706 (2011).

21. Herrick D.Z., Kuo W, Huang H, Schwieters C. D., Ellena J. F., Cafiso D.S. Solution and membrane-bound conformations of the tandem C2A and C2B domains of synaptotagmin 1: Evidence for bilayer bridging. J. Mol. Biol. 390, 913-923 (2009).

22. Rizo J., Sudhof T. C. The Membrane Fusion Enigma: SNAREs, Sec1/Munc18 Proteins, and Their Accomplices-Guilty as Charged? Annu. Rev. Cell Dev. Biol. 28, 279-308 (2012).

23. Schiavo G., Benfenati F., Poulain B., Rossetto O., Polverino de Laureto P., DasGupta B. R., Montecucco C. Tetanus and botulinum-B neurotoxinsblock neurotransmitter release by proteolytic cleavage ofsynaptobrevin. Nature. 359, 832-835 (1992).

24. Blasi J., Chapman E. R., Yamasaki S., Binz T., Niemann H., Jahn R. Botulinum neurotoxin $\mathrm{C} 1$ blocksneurotransmitter release by means of cleaving HPC-1/syntaxin. EMBO J. 12, 4821-4828 (1993).

25. Hanson P. I., Roth R., Morisaki H., Jahn R., Heuser J. E. Structure and conformational changes in NSF and its membrane receptor complexes visualized by quick-freeze/deep-etch electron microscopy. Cell. 90, 523535 (1997).

26. Lin R. C., Scheller R.H. Mechanisms of synaptic vesicle exocytosis. Annu. Rev. Cell Dev. Biol.16, 19-49 (2000).

27. Sutton R.B., Fasshauer D., Jahn R., Brunger A.T. Crystal structure of a SNARE complex involved in synaptic exocytosis at $2.4 \AA$ resolution. Nature. 395, 347-353 (1998).

28. Sollner T. H., Bennett M. K., Whiteheart S. W., Scheller R. H. Rothman, J. E. A protein assembly-disassembly pathway in vitro that may correspond to sequential steps of synaptic vesicle docking, activation, and fusion. Cell 75: 409-418 (1993).

29. Lin R. C., Scheller R. H. Structural organization of the synaptic exocytosis core complex. Neuron 19: 1087-94 (1997).

30. Hanson P. I., Heuser J. E., Jahn R. Neurotransmitter release - four years of SNARE complexes. Curr Opin Neurobiol 7, 310-315 (1997).

31. Jahn R., Scheller R. H., SNAREs-engines for membrane fusion. Nature reviews. 7, 631-643 (2006).

32. Ossig R., Schmitt H. D., de Groot B., Riedel D., Keränen S.,Ronne H., Grubmüller H., Jahn R. Exocytosis requires asymmetry in the central layer of the SNARE complex. EMBO J. 19, 6000-6010 (2000).

33. Fasshauer D., Sutton R. B., Brunger A.T., Jahn R. Conserved structural features of the synaptic fusion complex: SNARE proteins reclassified as Qand R-SNAREs. Proc. Natl Acad. Sci. 95, 15781-6 (1998).

34. Scales S. J., Yoo B.Y., Scheller R.H. The ionic layer is required for efficient dissociation of the SNARE complex by-SNAP and NSF. PNAS. 98, 142627 (2001). 
35. Fasshauer D., Eliason W. K, Brünger A. T., Jahn R. Identification of a Minimal Core of the Synaptic SNARE Complex Sufficient for Reversible Assembly and Disassembly. Biochemistry, 37, 10354-62 (1998).

36. Misura K. M., Scheller R. H., Weis W. I. Self-association of the H3 Region of Syntaxin 1A. J Biol Chem. 76, 13273-82 (2001).

37. Fernandez I., Ubach J., Dulubova I., Zhang X., Südhof T. C., Rizo J. ThreeDimensional Structure of an Evolutionarily Conserved N-Terminal Domain of Syntaxin 1A. Cell. 94, 841-849 (1998).

38. Lerman J. C., Robblee J., Fairman R., Hughson F. M. Structural Analysis of the Neuronal SNARE Protein Syntaxin-1A. Biochemistry. 25, 8470-9 (2000).

39. Dulubova I., Sugita S., Hill S., Hosaka M., Fernandez I., Südhof T. C., Rizo J. A conformational switch in syntaxin during exocytosis: role of Munc18. EMBO J. 18, 4372-4382 (1999).

40. Margittai M. J., Widengren J., Schweinberger E., Schröder G. F., Felekyan S., Haustein E., König M., Fasshauer D., Grubmüller H., Jahn R., Seidel C. A. M. Single-molecule fluorescence resonance energy transfer reveals a dynamic equilibrium between closed and open conformations of syntaxin 1 . Proc Natl Acad Sci. 100, 15516-21 (2003).

41. Chen X., Lu J., Dulubova I., Rizo J. NMR analysis of the closed conformation of syntaxin-1. J. Biomol. NMR. 41, 43-54 (2008).

42. Ma C., Li W., Xu Y., Rizo J. Munc13 mediates the transition from the closed syntaxin-Munc18 complex to the SNARE complex. Nature Structural \& Molecular Biology. 18, 542-549 (2011).

43. Misura K. M., Scheller R. H., Weis W. I. Three-dimensional structure of the neuronal Sec1-syntaxin 1a complex. Nature. 404, 355-62 (2000).

44. Rizo J. Sudhof T. C. SNAREs and Munc18 in synaptic vesicle fusion. Nature Reviews Neuroscience. 3, 641-653 (2002).

45. Rickman C., Medine C. N., Bergmann A., Duncan R. R. Functionally and spatially distinct modes of munc18-syntaxin 1 interaction. $J$ Biol Chem. 282, 12097-103 (2007).

46. Khvotchev M., Dulubova I., Sun J., Dai H., Rizo J., Südhof T. C. Dual modes of Munc18-1/SNARE interactions are coupled by functionally critical binding to syntaxin-1 N terminus. J Neurosci. 27, 12147-55 (2007).

47. Kroch A. E., Fleming K. G. Alternate Interfaces May Mediate Homomeric and Heteromeric Assembly in the Transmembrane Domains of SNARE Proteins. J Mol Biol. 357, 184-94 (2006).

48. Hanson P. I., Otto H., Barton N., Jahn R. The N-ethylmaleimide-sensitive fusion protein and alpha-SNAP induce a conformational change in syntaxin. J Biol Chem. 270, 16955-61 (1995).

49. Oyler G. A., Higgins G. A., Hart R. A., Battenberg E., Billingsley M., Bloom F. E., Wilson M. C. The identification of a novel synaptosomalassociated protein, SNAP-25, differentially expressed by neuronal subpopulations. J.Cell Biol. 109, 3039-3052 (1989).

50. $\quad$ Fasshauer D., Bruns D., Shen B., Jahn R., Brünger A. T. A structural change occurs upon binding of syntaxin to SNAP-25. J Biol Chem. 272, 4582-90 (1997). 
51. Weninger K., Bowen M. E., Choi U. B., Chu S., Brunger A. T. Accessory Proteins Stabilize the Acceptor Complex for Synaptobrevin, the 1:1 Syntaxin/SNAP-25 Complex. Structure. 16, 308-20 (2008).

52. Brewer K. D., Li W., Horne B. E., Rizo J. Reluctance to membrane binding enables accessibility of the synaptobrevin SNARE motif for SNARE complex formation. PNAS. 108, 12723-12728 (2011).

53. Bowen M., Brunger A. T. Conformation of the synaptobrevin transmembrane domain. PNAS. 103, 8378-83 (2006).

54. Ellena J. F., Liang B., Wiktor M., Stein A., Cafiso D.S., Jahn R., Tamm L.K. Dynamic structure of lipid-bound synaptobrevin suggests a nucleationpropagation mechanism for trans-SNARE complex formation. PNAS. 106. 20306-11 (2009).

55. Darios F., Wasser C., Shakirzyanova A., Giniatullin A., Goodman K., Munoz-Bravo J. L., Raingo J., Jorgacevski J., Kreft M., Zorec R., Rosa J. M., Gandia L., Gutiérrez L. M., Binz T., Giniatullin R., Kavalali E. T., Davletov B. Sphingosine Facilitates SNARE Complex Assembly and Activates Synaptic Vesicle Exocytosis. Neuron. 62, 683-694 (2009).

56. Walter A. M., Wiederhold K., Bruns D., Fasshauer D., Sørensen J. B. Synaptobrevin N-terminally bound to syntaxin-SNAP-25 defines the primed vesicle state in regulated exocytosis. JCB. 188, 401-413 (2010).

57. Pobbati A. V., Stein A., Fasshauer D. N- to C-Terminal SNARE Complex Assembly Promotes Rapid Membrane Fusion. Science. 313, 673-6 (2006).

58. Weber T., Zemelman B. V., McNew J. A., Westermann B., Gmachl M., Parlati F., Söllner T. H., Rothman J. E. SNAREpins: Minimal Machinery for Membrane Fusion. Cell. 92, 759-72 (1998).

59. Wiederhold K., Kloepper T. H., Walter A. M., Stein A., Kienle N., Sørensen J. B., Fasshauer D. A coiled coil trigger site is essential for rapid binding of synaptobrevin to the SNARE acceptor complex. J Biol Chem. 285, 2154959 (2010).

60. Fasshauer D. Margittai M. Transient N-terminal Interaction of SNAP-25 and Syntaxin Nucleates SNARE Assembly. Journal of Biological Chemistry, 279, 7613-7621 (2004).

61. Verhage M., Maia A. S., Plomp J. J., Brussaard A. B., Heeroma J. H., Vermeer H., Toonen R. F., Hammer R. E., van den Berg T. K., Missler M., Geuze H.J., Südhof T. C. Synaptic assembly of the brain in the absence of neurotransmitter secretion. Science. 287: 864 - 869 (2000).

62. Hata Y., Slaughter C. A., Südhof T. C. Synaptic vesicle fusion complex contains unc-18 homologue bound to syntaxin. Nature, 366, 347-351 (1993).

63. Burkhardt P., Hattendorf D. A., Weis W. I., Fasshauer D. Munc18a controls SNARE assembly through its interaction with the syntaxin N-peptide. EMBO J. 27, 923-33 (2008).

64. Schütz D., Zilly F., Lang T., Jahn R., Bruns D. A dual function for Munc-18 in exocytosis of PC12 cells. European Journal of Neuroscience. 21, 2419 $2432(2005)$. 
65. Dulubova I., Khvotchev M., Liu S., Huryeva I., Südhof T. C., Rizo J. Munc18-1 binds directly to the neuronal SNARE complex. Proc. Natl. Acad. Sci. 104, 2697-702 (2007).

66. Shen J., Tareste D. C., Paumet F., Rothman J. E., Melia T. J. Selective activation of cognate SNAREpins by Sec1/Munc18 proteins. Cell, 128, 183-95 (2007).

67. Richmond J. E., Davis W. S., Jorgensen E. M. UNC-13 is required for synaptic vesicle fusion in C. elegans. Nat. Neurosci. 2, 959-964 (1999).

68. Varoqueaux F., Sigler A., Rhee J. S., Brose N., Enk C., Reim K., Rosenmund C. Total arrest of spontaneous and evoked synaptic transmission but normal synaptogenesis in the absence of Munc13-mediated vesicle priming. Proc. Natl. Acad. Sci. 99, 9037-42 (2002).

69. Aravamudan B., Fergestad T., Davis W. S., Rodesch C. K., Broadie K. Drosophila UNC-13 is essential for synaptic transmission. Nat. Neurosci. 2, 965-71 (1999).

70. Rhee JS., Betz A, Pyott S., Reim K., Varoqueaux F., Augustin I., Hesse D., Südhof T. C, Takahashi M., Rosenmund C., Brose N. $\beta$ Phorbol ester- and diacylglycerol-induced augmentation of transmitter release is mediated by Munc13s and not by PKCs. Cell. 108, 121-33 (2002).

71. Shin OH., Lu J., Rhee J. S., Tomchick D. R., Pang Z. P., Wojcik S. M., Camacho-Perez M., Brose N., Machius M., Rizo J., Rosenmund C., Südhof T. C. Munc13 C2B domain is an activity-dependent $\mathrm{Ca}^{2+}$ regulator of synaptic exocytosis. Nat Struct Mol Biol. 17, 280-8 (2010).

72. Guan R., Dai H., Rizo J. Binding of the Munc13-1 MUN domain to membrane-anchored SNARE complexes. Biochemistry. 47, 1474-81 (2008).

73. Ma C., Su L, Seven A., B, Xu Y., Rizo J. Reconstitution of the Vital Functions of Munc18 and Munc13 in Neurotransmitter Release. Science. 39, 421-425 (2013).

74. Richmond J. E., Weimer R. M., Jorgensen E. M. An open form of syntaxin bypasses the requirement for UNC-13 in vesicle priming. Nature. 412,338341 (2001).

75. Rizo J., Chen X., Arac D. Unraveling the mechanisms of synaptotagmin and SNARE function in neurotransmitter release.Trends in Cell Biology .16: 339-350 (2006).

76. Schreier S., Polnaszek C. F., Smith I. C. Spin labels in membranes. Problems in practice. Biochim. Biophys. Acta. 515, 395-436 (1978).

77. Schleight H., Spiess H. W. Jeschke G. A site-directed spin-labeling study of surfactants in polymer-clay nanocomposites. Colloid Polym Sci. 284, 121119 (2006).

78. Hubbell W. L., Cafiso D. S., Altenbach, C. Identifying conformational changes with site-directed spin labeling. Nature Structural Biology 7: 735739 (2000).

79. Budil DE, Lee S, Saxena S, Freed J. H. Nonlinear-Least-Squares Analysis of Slow-Motion EPR Spectra in One and Two Dimensions Using a Modified Levenberg-Marquardt Algorithm. J. Magn. Reson., 120, 155-189 (1996). 
80. Mchaourab H. S., Lietzow M. A., Hideg K., Hubbell W. L. Motion of spinlabeled side chains in T4 lysozyme: Correlation with protein structure and dynamics. Biochemistry. 35, 7692-704 (1996).

81. Reginsson G. W., Schiemann O. Pulsed electron-electron double resonance: beyond nanometre distance measurements on biomacromolecules. Biochem. J. 434. 353-363 (2011).

82. Jeschke G. Distance Measurements in the Nanometer Range by Pulse EPR, Chem Phys Chem. 3, 927-932 (2002).

83. Boura E., Rózycki B., Herrick D. Z., Chung H. S., Vecer J., Eaton W. A., Cafiso D. S., Hummer G., Hurley J. H. Solution structure of the ESCRT-I complex by small-angle X-ray scattering, EPR, and FRET spectroscopy, Proc. Natl. Acad. Sci. 108. 9437-42 (2011).

84. Endeward B., Butterwick J. A., MacKinnon R., Prisner T. F. Pulsed Electron-Electron Double-Resonance Determination of Spin-Label Distances and Orientations on the Tetrameric Potassium Ion Channel KcsA, J. Am. Chem. Soc. 131, 15246-50 (2009).

85. Pornsuwan S., Bird G., Schafmeister C. E., Saxena, S. Flexibility and lengths of bis-peptide nanostructures by electron spin resonance. $\mathrm{J}$. Am. Chem. Soc. 128, 3876-3877 (2006).

86. Kuznetsov N. A., Milov A. D., Koval V. V., Samoilova R. I., Grishin Y. A., Knorre D. G., Tsvetkov Y. D., Fedorova O. S., Dzuba S. A. PELDOR study of conformations of double-spin-labeled single- and double-stranded DNA with non-nucleotide inserts, Phys. Chem. Chem. Phys. 11, 6826-6832 (2009).

87. E580 Pulse EPR Training Course, Bruker

88. Poole, C. P. Electron Spin Resonance a Comprehensive Treatise on Experimental Techniques, Dover Publications, Inc., Mineola, New York. 1983.

89. Liu Hemminga M. A., Berliner L. J. ESR spectroscopy in membrane biophysics. Springer. 2007.

90. Berliner L. J., Eaton S. S., Eaton G. R. Distance Measurements in Biological Systems by EPR. Biological Magnetic Resonance. New York, Kluwer Academic. 2001.

91. Ghimire H., McCarrick R. M., Budil D. E., Lorigan G. A. Significantly improved sensitivity of Q-band PELDOR/DEER experiments relative to Xband is observed in measuring the intercoil distance of a leucine zipper motif peptide (GCN4-LZ). Biochemistry. 48, 5782-4 ( 2009).

92. Fleissner MR., Bridges M. D., Brooks E. K., Cascio D., Kálai T., Hideg K., Hubbell W. L. Structure and dynamics of a conformationally constrained nitroxide side chain and applications in EPR spectroscopy. Proc Natl Acad Sci. 108, 16241-6 (2011).

93. Fawzi N. L., Fleissner M. R., Anthis N. J., Kálai T., Hideg K., Hubbell W. L., Clore G. M. A rigid disulfide-linked nitroxide side chain simplifies the quantitative analysis of PRE data. J Biomol NMR. 51, 105-14 (2011).

94. Malmberg N. J., Falke J. J. Use of EPR Power Saturation to Analyze the Membrane-Docking Geometries of Peripheral Proteins: Applications to C2 Domains. Annu Rev Biophys Biomol Struct. 34, 71-90 (2005). 
95. Altenbach C., Greenhalgh D. A., Khorana H. G., Hubbell W. L. A collision gradient method to determine the immersion depth of nitroxides in lipid bilayers: Application to spin-labeled mutants of bacteriorhodopsin. Proceedings of the National Academy of Sciences. 91, 1667-1671 (1994).

96. Krzystek J., Sienkiewicz A., Pardi L., Brunel L. C. DPPH as a Standard for High-Field EPR. Journal of Magnetic Resonance. 125, 207-211 (1997).

97. Landgraf K., Malmberg N., Falke J. Effect of PIP 2 Binding on the Membrane Docking Geometry of PKC R C2 Domain: An EPR SiteDirected Spin-Labeling and Relaxation Study. Biochemistry. 47, 8301-8316 (2008).

98. Jahn R., Lang T., Sudhof T. Membrane Fusion Review. Cell, 112, 519533 (2003).

99. Columbus L., Hubbell W.L. A new spin on protein dynamics. Trends Biochem Sci. 27, 288-95 (2002).

100. http://rmn10.qfa.uam.es/epr/tutorial_uk/5_1Radicals_containing_n_eq.htmt

101. Hong, W. SNAREs and traffic. Biochim. Biophys. Acta. 1744, 493517 (2005).

102. Toonen, R. F., and M. Verhage. Munc18-1 in secretion: lonely Munc joins SNARE team and takes control. Trends Neurosci. 30, 564-572 (2007).

103. Carr, C. M., and J. Rizo. At the junction of SNARE and SMprotein function. Curr. Opin. Cell Biol. 22, 488-495 (2010)..

104. Meijer, M., P. Burkhardt, M. Verhage. Munc18-1 mutations that strongly impair SNARE-complex binding support normal synaptic transmission. EMBO J. 31, 2156-2168 (2012).

105. Christie M. P., Whitten A. E., King G. J., Hu S. H., Jarrott R. J., Chen K. E., Duff A. P., Callow P., Collins B. M., James D. E., Martin J. L. Lowresolution solution structures of Munc18:Syntaxin protein complexes indicate an open binding mode driven by the Syntaxin N-peptide. Proc Natl Acad Sci. 109, 9816-21 (2012).

106. Mittag, T., L. E. Kay, and J. D. Forman-Kay.. Protein dynamics and conformational disorder in molecular recognition. J. Mol. Recognit. 23, 105- 116 (2010)

107. Trizac E., Levt Y., Wolynes P. Capillarity theory for the fly-casting mechanism. Proc Natl Acad Sci. 107, 2746-2750 (2010).

108. Flores Jiménez R. H., Do Cao M. A., Kim M., Cafiso D. S. Osmolytes modulate conformational exchange in solvent-exposed regions of membrane proteins. Protein Sci. 19, 269-278 (2010).

109. Lopez, C. J., M. R. Fleissner, W. L. Hubbell. Osmolyte perturbation reveals conformational equilibria in spin-labeled proteins. Protein Sci. 18, 16371652 (2009).

110. McCoy, J., and W. L. Hubbell. High-pressure EPR reveals conformational equilibria and volumetric properties of spin-labeled proteins. Proc. Natl. Acad. Sci. 108, 1331-1336 (2011)..

111. Pannier, M., S. Veit, H. W. Spiess.. Dead-time free measurement of dipoledipole interactions between electron spins. J. Magn. Reson. 142, 331-340 (2000). 
112. Jeschke, G., V. Chechik, H. Jung. DeerAnalysis2006 - a comprehensive software package for analyzing pulsed ELDOR data. Appl. Magn. Reson. 30, 473-498 (2006).

113. Schwieters C. D., Kuszewski J. J., Tjandra N., Clore G. M. Using XplorNIH for NMR Molecular Structure Determination. Progress in Nuclear Magnetic Resonance Spectroscopy. 48, 47-62 (2006).

114. Schwieters C. D., Kuszewski J. J., Tjandra N., Clore G. M. The Xplor-NIH Molecular Structure Determination Package. Journal of Magnetic Resonance. 160, 65-73 (2003).

115. Guo Z., Cascio D., Hideg K., Hubbell W. L. Structural determinants of nitroxide motion in spin-labeled proteins: solvent-exposed sites in helix B of T4 lysozyme. Protein Sci. 17, 228-239 (2008).

116. Guo Z., Cascio D., Hideg K., Kálái T., Hubbell W. L. Structural determinants of nitroxide motion in spin-labeled proteins: tertiary contact and solvent-inaccessible sites in helix G of T4 lysozyme. Protein Sci. 16, 1069-1086 (2008).

117. Margittai, M., D. Fasshauer, R. Langen. 2001. Homo- and heterooligomericSNARE complexes studied by site-directed spin labeling. J. Biol. Chem. 276, 13169-13177.

118. Lee, D., C. Hilty, K.Wuthrich. 2006. Effective rotational correlation times of proteins from NMR relaxation interference. J. Magn.Reson. 178, 72-76.

119. Hilger, D., H. Jung,. G. Jeschke. Assessing oligomerization of membrane proteins by four-pulse DEER: $\mathrm{pH}$-dependent dimerization of NhaA Nap/Hp antiporter of E. coli. Biophys. J. 89, 1328-1338 (2005).

120. Parsegian, V. A., R. P. Rand, and D. C. Rau. 2000. Osmotic stress, crowding, preferential hydration, and binding: A comparison of perspectives. Proc. Natl. Acad. Sci. 97, 3987-3992.

121. Rosgen, J., B. M. Pettitt, and D. W. Bolen. An analysis of the molecular origin of osmolyte-dependent protein stability. Protein Sci. 16, 733-743 (2007).

122. Timasheff, S. N. Protein hydration, thermodynamic binding, and preferential hydration. Biochemistry. 41, 13473-13482 (2002).

123. Kim, C. S., D. H. Kweon, and Y. K. Shin. Membrane topologies of neuronal SNARE folding intermediates. Biochemistry. 41, 10928-10933 (2002).

124. Columbus, L., and W. L. Hubbell. Mapping backbone dynamics in solution with site-directed spin labeling: GCN4-58 bZip free and bound to DNA. Biochemistry. 43, 7273-7287 (2004).

125. Van Holde, K. E. 1971. Physical biochemistry. Prentice-Hall, Englewood Cliffs, N.J.

126. Columbus, L., T. Kalai, J. Jeko, K. Hideg, and W. L. Hubbell. Molecular motion of spin labeled side chains in alpha-helices: analysis by variation of side chain structure. Biochemistry 40, 3828-3846 (2001).

127. Carr, C. M., and Rizo, J. At the junction of SNARE and SM protein function. Curr. Opin. Cell Biol. 22, 488-495 (2010).

128. Hazzard, J., Sudhof, T. C., and Rizo, J. NMR analysis ofthe structure of synaptobrevin and of its interaction with syntaxin. J.Biomol. NMR 14, 203-207 (1999). 
129. Schuette, C. G., Hatsuzawa, K., Margittai, M., Stein, A., Riedel,D., Kuster, P., Konig, M., Seidel, C., and Jahn, R. Determinants of liposome fusion mediated by synaptic SNARE proteins. Proc. Natl.Acad. Sci. U.S.A. 101, 2858-2863 (2004).

130. Lee, D., Walter, K. F., Bruckner, A. K., Hilty, C., Becker, S., and Griesinger, C. Bilayer in small bicelles revealed by lipid-protein interactions using NMR spectroscopy. J. Am. Chem. Soc. 130, 13822-13823 (2008).

131. Pervushin, K., Riek, R., Wider, G., and Wuthrich, K. Attenuated T-2 relaxation by mutual cancellation of dipole-dipole coupling and chemical shift anisotropy indicates an avenue to NMRstructures of very large biological macromolecules in solution. Proc. Natl. Acad. Sci. 94, 12366-12371 (1997).

132. Grzesiek, S., and Bax, A. Improved 3D triple-resonance NMR techniques applied to a 31-kDa protein. J. Magn. Reson. 96, 432-440 (1992).

133. Wittekind, M., and Mueller, L. HNCACB, a high sensitivity 3D NMR experiment to correlate amide-proton and nitrogen resonances with the $\alpha$ carbon and $\beta$-carbon resonances in proteins. J. Magn. Reson., 101, 201-205 (1993).

134. Clubb, R. T., Thanabal, V., and Wagner, G. A constant time 3-dimensional triple-resonance pulse scheme to correlate intraresidue $\mathrm{H}-1(\mathrm{~N}), \mathrm{N}-15$, and C-13(') chemical-shifts in N-15-C-13-labeled proteins. J. Magn. Reson. 97, 213-217 (1992).

135. Metzler, W. J., Constantine, K. L., Friedrichs, M. S., Bell, A. J.,Ernst, E. G., Lavoie, T. B., and Mueller, L. Characterization of the three-dimensional solution structure of human profilin: $1 \mathrm{H}, 13 \mathrm{C}$, and $15 \mathrm{~N}$ NMR assignments and global folding pattern. Biochemistry. 32, 13818-13829 (1993).

136. Kay, L. E., Torchia, D. A., and Bax, A. Backbone dynamics of proteins as studied by $15 \mathrm{~N}$ inverse detected heteronuclear NMR spectroscopy: Application to staphylococcal nuclease. Biochemistry. 28, 8972-8979 (1989).

137. Loria, J. P., Rance, M., and Palmer, A. G., A TROSY CPMG sequence for characterizing chemical exchange in large proteins. J. Biomol. NMR. 15, 151-155 (1999).

138. Mulder, F. A., Skrynnikov, N. R., Hon, B., Dahlquist, F. W., and Kay, L. E. Measurement of slow $(\mu \mathrm{s}-\mathrm{ms})$ time scale dynamics in protein side chains by 15N relaxation dispersion NMR spectroscopy: Application to Asn and Gln residues in a cavity mutant of T4 lysozyme. J. Am. Chem. Soc. 123, 967-975 (2001).

139. Delaglio, F., Grzesiek, S., Vuister, G. W., Zhu, G., Pfeifer, J., and Bax, A. NMRPipe: A multidimensional spectral processing system based on UNIX pipes. J. Biomol. NMR. 6, 277-293 (1995).

140. Goddard, T. D., and Kneller, D. G. SPARKY 3, University of California, San Francisco (2006).

141. Camilloni, C., De Simone, A., Vranken, W. F., and Vendruscolo, M. Determination of secondary structure populations in disordered states of proteins using nuclear magnetic resonance chemical shifts. Biochemistry. 51, 2224-2231 (2012). 
142. Marsh, J. A., Singh, V. K., Jia, Z. C., and Forman-Kay, J. D. Sensitivity of secondary structure propensities to sequence differences between $\alpha$ - and $\gamma$ synuclein: Implications for fibrillation. Protein Sci. 15, 2795-2804 (2006).

143. Kweon, D. H., Kim, C. S., and Shin, Y. K. Regulation of neuronal SNARE assembly by the membrane. Nat. Struct. Biol. 10,440-447 (2003).

144. Qin, Z., Wertz, S. L., Jacob, J., Savino, Y., and Cafiso, D. S. Defining protein-protein interactions using site-directed spin labeling: The binding of protein kinase C substrates to calmodulin. Biochemistry. 35, 13272-13276 ( 1996).

145. Victor, K., Jacob, J., and Cafiso, D. S. Interactions controlling the membrane binding of basic protein domains: Phenylalanine and the attachment of the myristoylated alanine-rich C-kinase substrate protein to interfaces. Biochemistry. 38, 12527-12536 (1999).

146. Subczynski W. K., Wisniewska A., Yin J. J., Hyde J. S., Kusumi A. Hydrophobic Barriers of Lipid Bilayer-Membranes Formed by Reduction of Water Penetration by Alkyl Chain Unsaturation and Cholesterol. Biochemistry. 33, 7670-7681 (1994).

147. Freed, D. M., Horanyi, P. S., Wiener, M. C., and Cafiso, D. S. Conformational exchange in a membrane transport protein is altered in protein crystals. Biophys. J. 99, 1604-1610 (2010).

148. Kroncke, B. M., Horanyi, P. S., and Columbus, L. Structural origins of nitroxide side chain dynamics on membrane protein $\alpha$-helical sites. Biochemistry. 49, 10045-10060 (2010).

149. López C. J., Oga S., Hubbell W. L. Mapping molecular flexibility of proteins with site-directed spin labeling: A case study of myoglobin. Biochemistry. 51, 6568-83 (2012).

150. Ladokhin, A. S., and White, S. H. Folding of amphipathic $\alpha$-helices on membranes: Energetics of helix formation by melittin. J. Mol. Biol. 285, 1363-1369 (1999).

151. Li, Y., Han, X., and Tamm, L. K. Thermodynamics of fusion peptidemembrane interactions. Biochemistry. 42, 7245-7251 (2003).

152. Seelig, J. Thermodynamics of lipid-peptide interactions. Biochim. Biophys. Acta. 1666, 40-50 (2004).

153. Drin G., Antonny B. Amphipathic helices and membrane curvature. Febs Lett. 584, 1840-7 (2010).

154. Vamparys L., Gautier R., Vanni S., Bennett W. F., Tieleman D. P., Antonny B., Etchebest C., Fuchs P. F. Conical lipids in flat bilayers induce packing defects similar to that induced by positive curvature. Biophys. J. 104, 585-593 (2013).

155. Vanni S., Vamparys L., Gautier R., Drin G., Etchebest C., Fuchs P. F., Antonny B. Amphipathic lipid packing sensor motifs: Probing bilayer defects with hydrophobic residues. Biophys. J. 104, 575-584 (2013).

156. Steinmetz, M. O., Jelesarov, I., Matousek, W. M., Honnappa, S., Jahnke, W., Missimer, J. H., Frank, S., Alexandrescu, A. T., and Kammerer, R. A. Molecular basis of coiled-coil formation. Proc. Natl. Acad. Sci. 104, 7062-7067 (2007). 
157. Liang, B., Kiessling, V., and Tamm, L. K. (2013) Pre-fusion structure of syntaxin 1A suggests pathway for folding into neuronal trans-SNARE complex fusion intermediate. Proc. Natl. Acad. Sci. 110, 19384-19389 (2013).

158. Martens, S., Kozlov, M. M., and McMahon, H. T. How synaptotagmin promotes membrane fusion. Science. 316, 1205-1208 (2007).

159. Lynch, K. L., Gerona, R. R., Kielar, D. M., Martens, S., McMahon, H. T., and Martin, T. F. Synaptotagmin-1 utilizes membrane bending and SNARE binding to drive fusion pore expansion. Mol. Biol. Cell. 19, 5093-5103 (2008).

160. Andersson, M., Ulmschneider, J. P., Ulmschneider, M. B., and White, S. H. Conformational states of melittin at a bilayer interface. Biophys. J. 104, L12-L14 (2013). 